\title{
Discrete Morse theory for cellular resolutions
}

\section{(Diskrete Morsetheorie für zelluläre Auflösungen)}

\author{
DISSERTATION \\ zur \\ Erlangung des Doktorgrades \\ der Naturwissenschaften \\ (Dr. rer. nat.) \\ dem \\ Fachbereich Mathematik und Informatik \\ der Philipps-Universität Marburg \\ vorgelegt von
}

Ekkehard Batzies

aus Heidelberg

Marburg/Lahn 2002 


\section{Contents}

1 Introduction 3

2 Cellular resolutions $\quad 9$

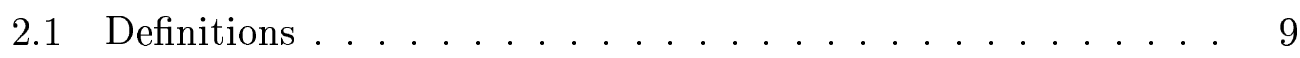

2.1.1 $\mathbb{Z}^{d}$-graded free resolutions $\ldots \ldots \ldots . \ldots 9$

2.1.2 CW-complexes . . . . . . . . . . 12

2.1.3 Cellular homology . . . . . . . . . . . . 15

2.1.4 Simplicial complexes . . . . . . . . . . . 18

2.1.5 Graded CW-complexes . . . . . . . . . . . 19

2.1.6 Cellular chain complexes and cellular resolutions . . . . 20

2.1.7 Co-Artinian monomial modules . . . . . . . . . . 21

2.2 Some basic facts about cellular resolutions . . . . . . . . . 22

2.2.1 A criterion for a CW-complex to support a cellular resolution .................. 23

2.2.2 Betti numbers from given resolutions . . . . . . . 24

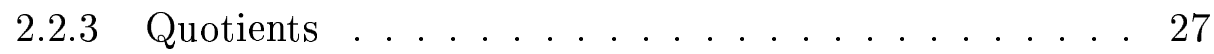

2.3 Examples of cellular resolutions . . . . . . . . . . 27

2.3.1 Taylor resolution . . . . . . . . . . . 27

2.3.2 Scarf resolution . . . . . . . . . . . 30

2.3.3 Hull resolution ... . . . . . . . . . . . . . . . . . . . . . . . . . .

2.3.4 Lyubeznik's resolution . . . . . . . . . . . 34

2.3.5 Resolutions via rooted complexes . . . . . . . . 35

2.3.6 LCM- and $\mathrm{LCM}^{*}$ - resolutions . . . . . . . . . 37

2.3.7 Hypersimplex-resolution of the maximal homogeneous ideal . . . . . . . . . . . . . . 40 
2.3.8 Bar-resolution . . . . . . . . . . . 42

3 Discrete Morse theory $\quad 44$

3.1 Discrete Morse theory for CW-complexes . . . . . . . . . . . 44

3.2 Discrete Morse theory for graded CW-complexes . . . . . . . 46

3.2.1 Acyclic matchings . . . . . . . . . . . 46

3.2.2 The correspondence between discrete Morse functions and acyclic matchings . . . . . . . . . . 49

3.2.3 Discrete Morse theory for graded CW-complexes . . . . 51

3.3 Discrete Morse theory for cellular resolutions . . . . . . . . . 58

3.4 Morse differentials . . . . . . . . . . . . . . 59

4 Applications $\quad 67$

4.1 Shellability .................... 67

4.2 The Lyubeznik Resolution . . . . . . . . . . . . . . . 76

4.2.1 Applications to Classes of Monomial Modules . . . . . 80

4.3 Hypersimplex resolution of powers of the maximal homogeneous ideal .................... 84

4.4 Resolutions via rooted complexes revisited . . . . . . . . . 90

4.5 p-Borel fixed ideals . . . . . . . . . . . . . . . . 91

4.6 Application to the Resolution of $k$ over Semigroup-Rings . . . 95

5 Deutsche Zusammenfassung $\quad 100$

5.1 Einleitung . . . . . . . . . . . . . . 100

5.2 Überblick über die Arbeit . . . . . . . . . . . . . . . . 101

5.3 Struktur der Arbeit . . . . . . . . . . . . . . . . 102

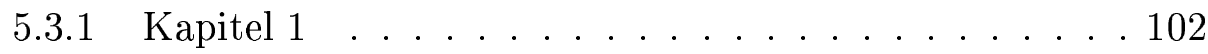

$5.3 .2 \quad$ Kapitel $2 \ldots \ldots \ldots \ldots . \ldots . \ldots . \ldots 102$

5.3 .3 Kapitel $3 \ldots \ldots \ldots . \ldots . \ldots . \ldots 107$

5.3 .4 Kapitel $4 \ldots \ldots \ldots . \ldots . \ldots 108 \ldots$ 


\section{Chapter 1}

\section{Introduction}

In linear algebra there is the fundamental concept of linear dependence. The situation turns out to be quite simple due to the fact that all vectorspaces $V$ over a field $k$ are free $k$-modules. In particular, a set of vectors $v_{1}, \ldots, v_{n} \in V$ is maximaly independent if and only if it is minimally generating.

The concept of dependence of polynomials $p_{1}, \ldots, p_{n} \in k\left[x_{1}, \ldots, x_{d}\right]$ over the polynomial ring $k\left[x_{1}, \ldots, x_{d}\right]$ is much more complex. Especially, maximal independence and minimal generation are not equivalent in this case.

In order to measure dependence we are led to the consideration of (minimal) free resolutions of the ideal $I=\left(p_{1}, \ldots, p_{n}\right)$ generated by the polynomials $p_{i}$. These are very difficult to find. It is even an open problem to construct minimal free resolutions of arbitrary monomial ideals $I$, that is, of ideals in the polynomial ring that are generated by monomials.

A reasonably well developed approach to this problem consists of studying so-called cellular resolutions. If the ideal under construction is $\mathbb{Z}^{d}$-graded these are formed by homogenizing the cellular chain complex of a $\mathbb{Z}^{d}$-graded CW-complex.

The classical example of a free $\mathbb{Z}^{d}$-graded cellular resolution is the Taylor resolution. The $\mathbb{Z}^{d}$-graded CW-complex supporting the Taylor resolution is the standard simplex $\Delta_{n}$ with vertices given by the monomial generators $m_{1}, \ldots, m_{n}$ of the ideal $I$. Here, $\Delta_{n}$ is $\mathbb{Z}^{d}$-graded by $\mathrm{lcm}$, that is, for all simplices $\sigma=\left\{m_{i_{1}}, \ldots, m_{i_{k}}\right\} \in \Delta_{n}$ the $\mathbb{Z}^{d}$-grading is given by $\alpha \in \mathbb{Z}^{d}$, where $\underline{\mathbf{x}}^{\alpha}=\operatorname{lcm}\left\{m_{i_{1}}, \ldots, m_{i_{k}}\right\}$. 
In general, cellular resolutions like the Taylor resolution are far from minimal. This thesis provides a general method to minimize given cellular $\mathbb{Z}^{d}$-graded free resolutions. For this, we use techniques from combinatorial topology, namely discrete Morse theory. The main feature of this theory developed by Forman $[13,14]$ is the following (here we use a version due to Chari [9]):

Given a CW-complex $X$ and an acyclic matching on the directed cell graph of $X$ there exists a CW-complex $X_{A}$ (called the Morse complex) that is homotopy equivalent to $X$ and the cells of which are in one-to-one correspondence with the $A$-critical cells of $X$. The transition from $X$ to $X_{A}$ can be regarded as a process of making the CW-decomposition of $X$ more efficient.

The central original idea of this thesis is to transfer this process from CWcomplexes to cellular free resolutions: It is shown that, given a cellular $\mathbb{Z}^{d_{-}}$ graded free resolution of a module $M$ supported by a $\mathbb{Z}^{d}$-graded CW-complex $X$ and a homogeneous acyclic matching $A$ on the directed cell graph of $X$, the Morse complex $X_{A}$ also supports a $\mathbb{Z}^{d}$-graded free resolution of $M$. Since the CW-complex $X_{A}$ consists of fewer cells than $X$ the cellular resolution supported by $X_{A}$ is smaller than the one supported by $X$.

Applying this method we are able to produce minimal $\mathbb{Z}^{d}$-graded free resolutions for certain classes of monomial modules. These include all generic, stable and squarefree stable monomial ideals, all Stanley-Reisner ideals corresponding to a simplicial complex with shellable Alexander dual as well as a very special class of p-Borel fixed ideals. Also, for a special class of affine semigroups $\Lambda \subset \mathbb{N}^{d}$, we are able to produce minimal $\mathbb{Z}^{d}$-graded free resolutions of the field $k$ over the affine semigroup ring $k[\Lambda]$.

The idea of applying discrete Morse theory to cellular resolutions has been presented before in [3] which is joint work with Volkmar Welker. Here, also the application to obtain a minimal Lyubeznik resolution for generic and shellable co-Artinian monomial modules as well as the cellular minimal $\mathbb{Z}^{d}$ graded resolution of the field $k$ over an affine semigroup ring $k[\Lambda]$ satisfying a certain condition of shellability can be found.

The structure of the thesis is as follows:

In Chapter 2, we set the stage by presenting definitions, basic facts and 
examples of cellular $\mathbb{Z}^{d}$-graded free resolutions. In particular, we present the well-known fact that the Betti-numbers of a $\mathbb{Z}^{d}$-graded module $M$ (that is, the exponents $\beta_{i}^{\alpha}$ of the free modules $\mathcal{F}_{i}=\bigoplus_{\alpha \in \mathbb{Z}^{d}} k\left[x_{1}, \ldots, x_{d}\right]^{\beta_{i}^{\alpha}}(-\alpha)$ appearing in the minimal $\mathbb{Z}^{d}$-graded free resolution of $M$ ) can be calculated from any given $\mathbb{Z}^{d}$-graded free resolution of $M$. We show that in consequence for a cellular $\mathbb{Z}^{d}$-graded free resolution of a $\mathbb{Z}^{d}$-graded module $M$ supported by a $\mathbb{Z}^{d}$-graded $\mathrm{CW}$-complex $X$ the Betti-numbers of $M$ can be calculated from the homology of the topological space $X$.

We list known examples of cellular $\mathbb{Z}^{d}$-graded free resolutions including the Taylor resolution (presented by Taylor in [23]), the Scarf resolution (Bayer, Peeva and Sturmfels, [4]), the Hull resolution (Bayer and Sturmfels, [5]) and resolutions via rooted complexes (Novik, [18]), show that certain known algebraic $\mathbb{Z}^{d}$-graded free resolutions like Lyubeznik's resolution (see [16]) and the Bar resolution are cellular and present new cellular $\mathbb{Z}^{d}$-graded free resolutions namely the LCM- and $\mathrm{LCM}^{*}$-resolutions and the hypersimplicial resolution for powers $\mathfrak{m}^{n}$ of the maximal ideal $\mathfrak{m} \subset k\left[x_{1}, \ldots, x_{d}\right]$.

Also, we show that quotients $M / N$ of co-Artinian monomial modules can be resolved using a relative version of the Taylor resolution.

In Chapter 3 we review discrete Morse theory and expand it to $\mathbb{Z}^{d}$-graded CW-complexes in order to obtain a discrete Morse theory for cellular resolutions. We follow the approach of Chari using acyclic matchings on the directed cell graph of the CW-complex $X$. In order to achieve the expansion to $\mathbb{Z}^{d}$-graded CW-complexes we give an explicit description of the Morse complex $X_{A}$.

In [13] Forman also presents a differential complex $\mathcal{M}$ generated freely by the critical cells of $X$ which he shows to be chain homotopic to the cellular chain complex of the CW-complex $X$. We show that, as expected, this differential complex actually coincides with the cellular chain complex of the Morse complex $X_{A}$, this way achieving explicit formulas for the maps in cellular $\mathbb{Z}^{d}$-graded free resolutions derived via discrete Morse theory for cellular resolutions.

Chapter 4 consists of applications:

1. We introduce a new class of monomial modules called shellable monomial modules. These include all stable and squarefree stable monomial 
modules as well as all Stanley-Reisner ideals corresponding to a simplicial complex with shellable Alexander dual. We present an application of discrete Morse theory for cellular resolutions to the $\mathrm{LCM}^{*}$-resolution of shellable co-Artinian monomial modules $M$ that yields a minimal cellular $\mathbb{Z}^{d}$-graded free resolution of $M$. Parallel to our work Sköldberg has constructed by algebraic means minimal free resolutions for a class of ideals that includes the shellable ones.

2. We provide a general method to apply discrete Morse theory for cellular resolutions to the Taylor resolution. The resulting resolution is called Lyubeznik resolution (since it can be regarded as a generalization of Lyubeznik's resolution). We show that for generic and shellable co-Artinian monomial modules there exist minimal Lyubeznik resolutions, this way presenting a second proof for the existence of a cellular minimal free $\mathbb{Z}^{d}$-graded resolution of shellable co-Artinian monomial modules. For generic monomial ideals the given resolution is the resolution determined by the Scarf-complex as shown by Bayer, Peeva \& Sturmfels (see [4]). For stable and squarefree-stable monomial ideals the minimal resolution had been determined by Eliahou \& Kervaire respectively Aramova, Herzog \& Hibi and Peeva by algebraic means (see [12] and [2]).

3. We give a application of discrete Morse theory for cellular resolutions to the hypersimplicial resolution for powers $\mathfrak{m}^{n}$ of the maximal ideal $\mathfrak{m} \subset$ $k\left[x_{1}, \ldots, x_{d}\right]$ which yields a cellular minimal $\mathbb{Z}^{d}$-graded free resolution of $\mathfrak{m}^{n}$. Using the formula for the differential of the Morse complex, we show that this minimal resolution is isomorphic to the one given by Eliahou and Kervaire (see [12]).

4. We show that the cellular resolution via rooted complexes can be obtained from the Taylor resolution via discrete Morse theory for cellular resolutions.

5. For p-Borel fixed ideals $I$ generated by a monomial $x_{d}^{r}$ (generated in the sense that $I$ is the smallest p-Borel fixed ideal such that $x_{d}^{r} \in I$ ) we show 
that an application of discrete Morse theory for cellular resolutions yields a cellular minimal $\mathbb{Z}^{d}$-graded free resolution of $I$.

6. We show that for an affine semigroup $\Lambda$ with the property that all order complexes $\Delta(\underline{\mathbf{0}}, \lambda), \lambda \in \Lambda$, over open intervalls $(\underline{\mathbf{0}}, \lambda)$ are shellable, there exists a cellular minimal $\mathbb{Z}^{d}$-graded free resolution of the field $k$ over the affine semigroup ring $k[\Lambda]$. This is achieved by an application of discrete Morse theory for cellular resolutions to the Bar-resolution of $k$ over $k[\Lambda]$.

Even though we have subsumed most cases of monomial ideals in which the minimal free resolution was known before and constructed new ones, still there are results not covered here. Notably in [19], Novik, Sturmfels and Postnikow give a beautiful geometric construction of polyhedral complexes minimally resolving matroid and oriented matroid ideals. We believe there is still large potential for combinatorial and geometric constructions in the theory of cellular free resolutions. 
I want to thank my advisor Volkmar Welker for excellent working support: He provided the original idea for this thesis and was open for discussions almost any minute. This way I was able to benefit from his great mathematical knowledge and intuition in a pleasurable and relaxed atmosphere. 


\section{Chapter 2}

\section{Cellular resolutions}

In this chapter we define the concept of a cellular resolution, which lays the ground for the application of discrete Morse theory in commutative algebra. Section 2.1 gives the definitions of free resolutions, CW-complexes, simplicial complexes, cellular free resolutions, monomial modules and co-Artinian monomial modules. Section 2.2 presents some basic facts about cellular resolutions. Section 2.3 lists some well-known and some new examples of cellular resolutions. In Chapter 4 these will act as starting points for applications of discrete Morse theory to cellular resolutions.

\subsection{Definitions}

\subsection{1 $\quad \mathbb{Z}^{d}$-graded free resolutions}

Throughout the thesis, we denote by $\mathbb{N}$ the integers including 0 .

Definition 2.1.1. $\quad$ 1. A ring $R$ is called $\mathbb{Z}^{d}$-graded if there is a direct sum decomposition

$$
R=\bigoplus_{\alpha \in \mathbb{Z}^{d}} R_{\alpha}
$$

of $R$ as abelian group with subgroups $R_{\alpha}, \alpha \in \mathbb{Z}^{d}$, such that

$$
R_{\alpha} R_{\beta} \subset R_{\alpha+\beta} \text {. }
$$


2. If $R=\bigoplus_{\alpha \in \mathbb{Z}^{d}} R_{\alpha}$ is a $\mathbb{Z}^{d}$-graded ring, the $R$-module $M$ is called $\mathbb{Z}^{d}$ graded if there is a direct sum decomposition

$$
M=\bigoplus_{\alpha \in \mathbb{Z}^{d}} M_{\alpha}
$$

as abelian groups such that

$$
R_{\alpha} M_{\beta} \subset M_{\alpha+\beta}
$$

If

$$
N=\bigoplus_{\alpha \in \mathbb{Z}^{d}} N_{\alpha}
$$

is another $\mathbb{Z}^{d}$-graded $R$-module and

$$
\phi: M \longrightarrow N
$$

is an $R$-linear map, we call $\phi$ homogeneous if for all $\alpha \in \mathbb{Z}^{d}$ we have

$$
\phi\left(M_{\alpha}\right) \subset N_{\alpha} .
$$

3. An affine semigroup $\Lambda$ is a finitely generated (i.e., $\Lambda=\mathbb{N} \lambda_{1}+\cdots+\mathbb{N} \lambda_{r}$ for some elements $\lambda_{1}, \ldots, \lambda_{r} \in \mathbb{N}^{d}$ ) sub-semigroup of the semigroup $\mathbb{N}^{d}$.

4. For a given affine semigroup $\Lambda$ we order $\mathbb{Z}^{d}$ by $\alpha \preceq \beta$ if and only if $\alpha+\gamma=\beta$ for some $\gamma \in \Lambda$. We write $\left(\mathbb{Z}^{d}, \Lambda\right)$ for the poset $\mathbb{Z}^{d}$ with the order induced by $\Lambda$.

5. Let $k$ be a field. A ring $R$ is called affine semigroup ring if $R=k[\Lambda]$ is the subring of $S:=k\left[x_{1}, \ldots, x_{d}\right]$ with $k$-basis given by the monomials $\underline{\mathbf{x}}^{\lambda}=x_{1}^{\lambda_{1}} \cdots x_{d}^{\lambda_{d}}$ for elements $\lambda=\left(\lambda_{1}, \ldots, \lambda_{d}\right) \in \Lambda$, where $\Lambda \subset \mathbb{N}^{d}$ is an affine semigroup. Note that $R=k[\Lambda]$ is naturally $\mathbb{Z}^{d}$-graded.

6. Let $R=k[\Lambda]$ be an affine semigroup ring. We denote by $\mathfrak{m} \subset k[\Lambda]$ the maximal homogeneous ideal in $k[\Lambda]$. This is the ideal generated by all monomials $\underline{\mathbf{x}}^{\lambda}=x_{1}^{\lambda_{1}} \cdots x_{d}^{\lambda_{d}}$ for elements $\lambda=\left(\lambda_{1}, \ldots, \lambda_{d}\right) \in$ $\Lambda-\{(0, \ldots, 0)\}$. 
For the majority of applications in this thesis this framework will be specialized to $\Lambda=\mathbb{N}^{d}$ and $R=S$. This is done by setting $r:=d$ and $\lambda_{i}:=e_{i}$, with $e_{i}$ the i-th standard unit vector in $\mathbb{N}^{d}$. For $\Lambda=\mathbb{N}^{d}$ the partial order as defined above is the usual partial order given by $\alpha \preceq \beta$ if and only if $\alpha_{i} \leq \beta_{i}$ for all $i=1, \ldots, d$. The more general framework is used for resolutions of the field $k$ over an affine semigroup ring $k[\Lambda]$.

Definition 2.1.2 ( $\mathbb{Z}^{d}$-graded free chain complex). Let $R$ be a $\mathbb{Z}^{d}$-graded ring, $\mathcal{C}_{i}$ for all $i \in \mathbb{N}$ a free $\mathbb{Z}^{d}$-graded $R$-module and

$$
\mathcal{C}=\ldots \stackrel{\partial_{i+1}}{\longrightarrow} \mathcal{C}_{i} \stackrel{\partial_{i}}{\longrightarrow} \mathcal{C}_{i-1} \stackrel{\partial_{i-1}}{\longrightarrow} \ldots \stackrel{\partial_{1}}{\longrightarrow} \mathcal{C}_{0}
$$

a sequence such that all maps $\partial_{i}: \mathcal{C}_{i} \longrightarrow \mathcal{C}_{i-1}, i \in \mathbb{N}-\{0\}$, are $\mathbb{Z}^{d_{-}}$ homogeneous and $\partial_{i} \circ \partial_{i+1}=0$ for all $i \in \mathbb{N}-\{0\}$. Then $\mathcal{C}$ is called a $\mathbb{Z}^{d}$-graded free chain complex (over $R$ ).

Remark 2.1.3. Note that if $R=k[\Lambda]$ is an affine semigroup ring and

$$
\mathcal{C}=\ldots \stackrel{\partial_{i+1}}{\longrightarrow} \mathcal{C}_{i} \stackrel{\partial_{i}}{\longrightarrow} \mathcal{C}_{i-1} \stackrel{\partial_{i-1}}{\longrightarrow} \ldots \stackrel{\partial_{1}}{\longrightarrow} \mathcal{C}_{0}
$$

is a $\mathbb{Z}^{d}$-graded free chain complex over $R$ then $\mathcal{C}_{i}=\bigoplus_{\alpha \in \mathbb{Z}^{d}} C_{i}^{\alpha}$ where $C_{i}^{\alpha}=$ $R(-\alpha)^{\beta_{i}^{\alpha}}$ is the free $R$-module of rank $\beta_{i}^{\alpha}$ with generators in multidegree $\alpha$.

Definition 2.1.4 ( $\mathbb{Z}^{d}$-graded free resolution). Let $R=k[\Lambda]$ be an affine semigroup ring. Let $M=\bigoplus_{\alpha \in \mathbb{Z}^{d}} M_{\alpha}$ be a $\mathbb{Z}^{d}$-graded $R$-module. A $\mathbb{Z}^{d}$-graded free resolution $\mathcal{F}$ of $M$ is a $\mathbb{Z}^{d}$-graded free chain complex

$$
\mathcal{F}=\ldots \stackrel{\partial_{i+1}}{\longrightarrow} \mathcal{F}_{i} \stackrel{\partial_{i}}{\longrightarrow} \mathcal{F}_{i-1} \stackrel{\partial_{i-1}}{\longrightarrow} \ldots \stackrel{\partial_{1}}{\longrightarrow} \mathcal{F}_{0}
$$

over $R$, satisfying the following properties:

1. $\mathcal{F}$ is exact, that is,

$$
\operatorname{Ker} \partial_{i}=\operatorname{Im} \partial_{i+1} \text { for all } i \in \mathbb{N}-\{0\},
$$

2. Coker $\partial_{1} \cong M$. 
Remark 2.1.5. Let $R=k[\Lambda]$ be an affine semigroup ring and

$$
\mathcal{F}=\ldots \stackrel{\partial_{i+1}}{\longrightarrow} \mathcal{F}_{i} \stackrel{\partial_{i}}{\longrightarrow} \mathcal{F}_{i-1} \stackrel{\partial_{i-1}}{\longrightarrow} \ldots \stackrel{\partial_{1}}{\longrightarrow} \mathcal{F}_{0}
$$

a $\mathbb{Z}^{d}$-graded free chain complex. Then $\mathcal{F}$ is a free resolution of the $\mathbb{Z}^{d}$-graded $R$-module $M$ if and only if there is a homogeneous map $\partial_{0}: \mathcal{F}_{0} \rightarrow M$, such that

$$
\ldots \stackrel{\partial_{i+1}}{\longrightarrow} \mathcal{F}_{i} \stackrel{\partial_{i}}{\longrightarrow} \mathcal{F}_{i-1} \stackrel{\partial_{i-1}}{\longrightarrow} \ldots \stackrel{\partial_{1}}{\longrightarrow} \mathcal{F}_{0} \stackrel{\partial_{0}}{\longrightarrow} M \rightarrow 0
$$

is exact.

Definition 2.1.6 (Minimality of a $\mathbb{Z}^{d}$-graded free resolution).

A $\mathbb{Z}^{d}$-graded free resolution

$$
\mathcal{F}=\ldots \stackrel{\partial_{i+1}}{\longrightarrow} \mathcal{F}_{i} \stackrel{\partial_{i}}{\longrightarrow} \mathcal{F}_{i-1} \stackrel{\partial_{i-1}}{\longrightarrow} \ldots \stackrel{\partial_{1}}{\longrightarrow} \mathcal{F}_{0},
$$

$\mathcal{F}_{i}=\bigoplus_{\alpha \in \mathbb{Z}^{d}} F_{i}^{\alpha}, F_{i}^{\alpha}=R(-\alpha)^{\beta_{i}^{\alpha}}$ of the $\mathbb{Z}^{d}$-graded $R$-module $M$ is called minimal if for all $i \in \mathbb{N}-\{0\}$ the image of $\partial_{i}$ is contained in $\mathfrak{m} \mathcal{F}_{i-1}$. The numbers

$$
\beta_{i}^{\alpha}(M):=\beta_{i}^{\alpha}
$$

are called the Betti numbers of $M$.

Remark 2.1.7. Existence and uniqueness up to chain-complex isomorphisms of the minimal free resolution are well known facts (see [11]) from commutative algebra. The term minimal comes from the fact that a minimal resolution

$$
\ldots \stackrel{\partial_{i+1}}{\longrightarrow} \mathcal{F}_{i} \stackrel{\partial_{i}}{\longrightarrow} \mathcal{F}_{i-1} \stackrel{\partial_{i-1}}{\longrightarrow} \ldots \stackrel{\partial_{1}}{\longrightarrow} \mathcal{F}_{0}
$$

of the $R$-module $M$ simultaneously assumes minimal values for all ranks $\beta_{i}^{\alpha}$, where $\mathcal{F}_{i}=\bigoplus_{\alpha \in \mathbb{Z}^{d}} F_{i}^{\alpha}$ and $F_{i}^{\alpha}=R(-\alpha)^{\beta_{i}^{\alpha}}$.

\subsubsection{CW-complexes}

Definition 2.1.8. A topological space is called an (open) cell of dimension $d$ (or $d$-cell) if it is homeomorphic to the $d$-dimensional open ball

$$
\stackrel{\circ}{B^{d}}=\left\{x=\left(x_{1}, \ldots, x_{d}\right) \in \mathbb{R}^{d} \mid \sum_{i=1}^{d} x_{i}^{2}<1\right\} .
$$


Definition 2.1.9 (CW-complex). We call a topological space $X$ a $C W$ complex, if there exists a collection $X^{(*)}=\left\{\sigma_{i} \mid i \in I\right\}$ of disjoint open cells such that

$$
X=\bigcup_{i \in I} \sigma_{i}
$$

satisfying the following properties:

1. $X$ is Hausdorff,

2. for every open cell $\sigma \in X^{(*)}$ of dimension $d$, there exists a continuous map

$$
f_{\sigma}: B^{d}:=\left\{x=\left(x_{1}, \ldots, x_{d}\right) \in \mathbb{R}^{d} \mid \sum_{i=1}^{d} x_{i}^{2} \leq 1\right\} \longrightarrow X
$$

such that the restriction $f_{\sigma}^{\circ}:=\left.f_{\sigma}\right|_{B^{d}}$ is a homeomorphism

$$
f_{\sigma}^{\circ}: \stackrel{\circ}{d} \stackrel{\cong}{\longrightarrow} \sigma
$$

and such that $f_{\sigma}\left(S^{d-1}\right)$ intersects only finitely many cells nontrivially, all of which have dimension at most $d-1$,

3. a subset $A \subset X$ is closed in $X$ if and only if $A \cap \bar{\sigma}$ is closed in $\bar{\sigma}$ for all $\sigma \in X^{(*)}$

For a cell $\sigma \in X^{(*)}$, we call the map

$$
f_{\sigma}: B^{d} \longrightarrow X
$$

the characteristic map of $\sigma$ and $\bar{\sigma}=f_{\sigma}\left(B^{d}\right)$ the closed cell that belongs to $\sigma$.

For $d \in \mathbb{N}$ we denote by $X^{d} \subset X$ the union of all cells of dimension at most $d$ and call $X^{d}$ the $d$-skeleton of $X$. By $X^{(d)}$ we denote the set of all cells of dimension $d$.

A subcomplex of $X$ is given by a subset of the cells of $X$ that forms a CW-complex with the same characteristic maps. We say $(X, A)$ is a pair of $C W$-complexes (or $C W$-pair) if $X$ is a CW-complex and $A$ a subcomplex of $X$. 
If $(X, A)$ is a pair of CW-complexes then we denote by $(X, A)^{(d)}$ the set of $d$-cells of $X$ that do not belong to $A$. We set $(X, A)^{(*)}:=\bigcup_{d \geq 0}(X, A)^{(d)}$.

There is a natural way to view $(X, A)^{(*)}\left(X^{(*)}\right.$ respectively) as a partially ordered set: For cells $\sigma^{\prime}, \sigma \in(X, A)^{(*)}\left(X^{(*)}\right.$ respectively) we set $\sigma^{\prime} \leq \sigma$ if and only if the closed cell $\overline{\sigma^{\prime}}$ is a subset of the closed cell $\bar{\sigma}$. We say that $\sigma^{\prime}$ is a facet of $\sigma$ if $\sigma^{\prime} \neq \sigma$ and for $\tau \in(X, A)^{(*)}$ the inclusion $\sigma^{\prime} \leq \tau \leq \sigma$ implies $\tau \in\left\{\sigma, \sigma^{\prime}\right\}$. We say that $\sigma$ is a facet of $X$ if $\sigma$ is maximal with respect to the above partial relation on $X^{(*)}$.

Remark 2.1.10. 1. Note that in all constructions mentioned above, a single $\mathrm{CW}$-complex $X$ can also be regarded as the $\mathrm{CW}$-pair $(X, \emptyset)$. In the remainder of this thesis we will sometimes implicitly identify $X$ with $(X, \emptyset)$.

2. The letters "CW" in the term "CW-complex" stand for "closure - finiteness" (refering to property 2.) and "weak topology" (refering to property 3.).

Definition 2.1.11. If $X$ and $Y$ are topological spaces, $A \subset X$ closed, $f$ : $A \longrightarrow Y$ a continuous map, we denote by $X \cup_{f} Y$ the quotient space of the disjoint union $X \dot{\cup} Y$ by the equivalence relation that is generated by the relations $a \sim f(a)$ for all $a \in A . \quad X \cup_{f} Y$ is endowed with the quotient topology induced by this equivalence relation.

Lemma 2.1.12. A topological space $X$ is a $C W$-complex if and only if it can be obtained by the following process of attaching cells of increasing dimensions:

Let $X^{0}$ be a discrete set of points. These points form the 0-skeleton. Assume the $d-1$-skeleton $X^{d-1}$ is constructed. Let $\dot{\bigcup}_{\sigma \in I_{d}} B_{\sigma}^{d}$ be a disjoint union of balls $B_{\sigma}^{d}=B^{d} \times\{\sigma\}$ of dimension $d, \sigma$ ranging over some index set $I_{d}$. For all $\sigma \in I_{d}$ let $f_{\partial \sigma}: S^{d-1}=\partial B^{d} \longrightarrow X^{d-1}$ be a continuous map. These maps induce a continuous map

$$
f: \bigcup_{\sigma \in I_{d}} S_{\sigma}^{d-1}=\partial\left(\bigcup_{\sigma \in I_{d}} B_{\sigma}^{d}\right) \longrightarrow X^{d-1}
$$

$S_{\sigma}^{d-1}$ denoting the sphere that bounds $B_{\sigma}^{d}$. We construct the d-skeleton of $X$ by setting

$$
X^{d}:=\left(\bigcup_{\sigma \in I_{d}} B_{\sigma}^{d}\right) \cup_{f} X^{d-1}
$$


Note that the map $f$ carries the information of how the d-dimensional balls $B_{\sigma}^{d}$ are attached by their boundaries $S_{\sigma}^{d-1}$ to $X^{d-1}$. The map $f_{\partial \sigma}$ is called the attaching map of $\sigma$.

We set

$$
X:=\bigcup_{d \in \mathbb{N}} X^{d}
$$

We define the topology of $X$ by setting for $A \subset X: A$ is closed in $X$ if and only if $A \cap X^{d}$ is closed in $X^{d}$ for all $d \in \mathbb{N}$.

Proof. If a topological space $X$ is given by a construction as described above, the cells of $X$ are in one-to-one correspondence with the elements of $I:=$ $\bigcup_{d \in \mathbb{N}} I_{d}$ : The characteristic map $f_{\sigma}$ of the cell corresponding to the index $\sigma \in I_{d}$ is given by the composition

$$
B^{d} \stackrel{\cong}{\longrightarrow} B_{\sigma}^{d c} \longrightarrow \bigcup_{\sigma \in I_{d}} B_{\sigma}^{d} \longrightarrow\left(\bigcup_{\sigma \in I_{d}} B_{\sigma}^{d}\right) \cup_{f} X^{d-1} \longrightarrow X .
$$

The cell itself is given by $f_{\sigma}\left(\stackrel{\circ}{B^{d}}\right)$. On the other hand, if $X$ is a CW-complex and $\sigma \in X^{(*)}$ a cell, $f_{\sigma}: B^{d} \longrightarrow X$ its characteristic map, we define the corresponding attaching map by

$$
f_{\partial \sigma}:=\left.f_{\sigma}\right|_{S^{d-1}}: S^{d-1}=\partial B^{d} \longrightarrow X^{d-1}:
$$

Using the properties of the quotient map

$$
\bigcup_{\sigma \in I_{d}} B_{\sigma}^{d} \dot{\cup} X^{d-1} \longrightarrow\left(\bigcup_{\sigma \in I_{d}} B_{\sigma}^{d}\right) \cup_{f} X^{d-1},
$$

it is straightforward to check that the identity map between two copies of $X$, one endowed with the topology according to the definition of the CWcomplex and one with that according to the attaching construction, is continuous in both directions. This proves the assertion.

\subsubsection{Cellular homology}

For a pair $(X, A)$ of $\mathrm{CW}$-complexes there is an elegant way to compute singular homology with coefficients in $k$ (see [8]): 
Definition 2.1.13 (Cellular chain complex). Let $(X, A)$ be a CW-pair. For $i \in \mathbb{N}$ let $\mathcal{C}_{i}(X, A)$ be the $k$-vectorspace freely generated by the $i$-cells of $X$ that do not lie in $A$. The following construction defines the differentials of a chain complex

$$
\mathcal{C}(X, A)=\ldots \stackrel{\partial_{i+1}}{\longrightarrow} \mathcal{C}_{i}(X, A) \stackrel{\partial_{i}}{\longrightarrow} \mathcal{C}_{i-1}(X, A) \stackrel{\partial_{i-1}}{\longrightarrow} \ldots \stackrel{\partial_{1}}{\longrightarrow} \mathcal{C}_{0}(X, A) \stackrel{0}{\longrightarrow} 0:
$$

called the cellular chain complex of the pair $(X, A)$.

For $i \in \mathbb{N}$ let $\pi_{i}: X^{i} \rightarrow X^{i} / X^{i-1}$ be the canonical projection. Note that

$$
X^{i} / X^{i-1} \cong \bigvee_{\sigma \in X^{(i)}} S_{\sigma}^{i}
$$

with $S_{\sigma}^{i}=S^{i} \times\{\sigma\}$ denoting a sphere representing the image of the closed cell $f_{\sigma}\left(B_{\sigma}^{i}\right)$ under the projection $\pi_{i}$. Here the wedge is taken by choosing a base point $* \in S^{i}$ and by identifying all $(*, \sigma), \sigma \in X^{(i)}$.

For $i \in \mathbb{N}$ let $\gamma_{i}: B^{i} \longrightarrow S^{i}$ be any continuous map collapsing the bordering sphere $S^{i-1}$ of $B^{i}$ to the base point of $S^{i}$ such that the restriction to the interior of $B^{i}$ is a homeomorphism onto its image. For $\sigma \in(X, A)^{(i)}$ define $p_{\sigma}: X^{i} \rightarrow S^{i}$ such that $p_{\sigma} \circ f_{\sigma}=\gamma_{i}$ and $p_{\sigma} \circ f_{\sigma^{\prime}}$ is the constant map onto the base point of $S^{i}$ for all $\sigma^{\prime} \in(X, A)^{(i)}, \sigma \neq \sigma^{\prime}$. We call $p_{\sigma}$ the cellular projection map corresponding to the cell $\sigma$.

Recall that for a map $f: S^{i} \longrightarrow S^{i}$ it is common to define the degree $\operatorname{deg} f$ to be the unique integer such that the induced homomorphism in homology $f_{*}: \tilde{H}_{i}\left(S^{i} ; \mathbb{Z}\right) \longrightarrow \tilde{H}_{i}\left(S^{i} ; \mathbb{Z}\right)$ is multiplication with $\operatorname{deg} f$.

Let $\sigma$ be an $i$-cell. We set

$$
\partial_{i}(\sigma):=\sum_{\tau \in X^{(i-1)}}[\tau: \sigma] \tau
$$

with

$$
[\tau: \sigma]:=\operatorname{deg}\left(p_{\tau} \circ f_{\partial \sigma}\right) .
$$

This defines the differential $\partial_{i}: \mathcal{C}_{i}(X, A) \longrightarrow \mathcal{C}_{i-1}(X, A)$.

Theorem 2.1.14. (see [8], p. 204) The singular homology $H_{*}(X, A)$ of the pair $(X, A)$ is isomorphic to the homology of the chain complex $\mathcal{C}(X, A)$. 


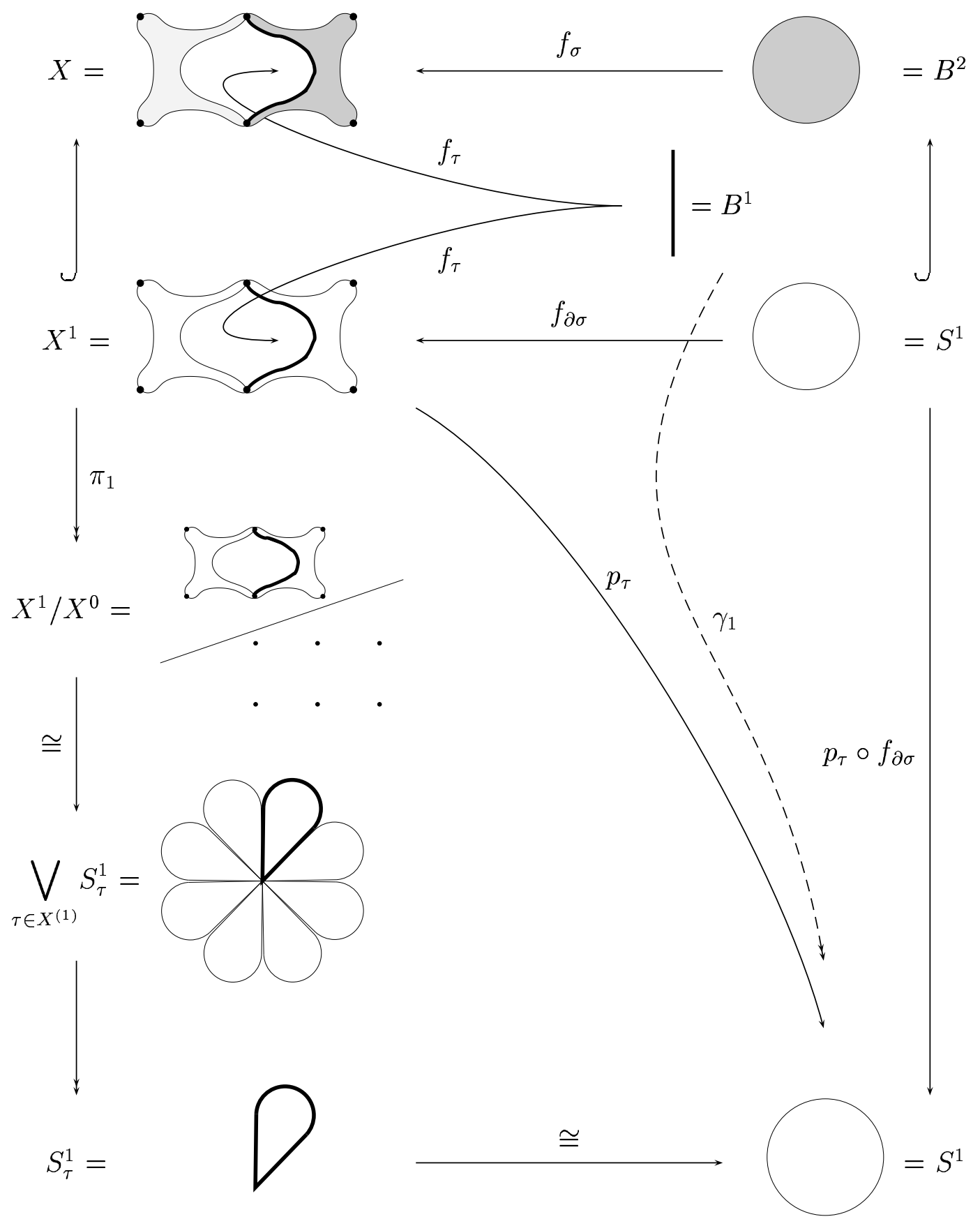

Figure 1.

Example of a 2-dimensional CW-complex $X$ with two maximal cells, both of dimension 2. The righthand one is denoted by $\sigma$. The figure illustrates 
how to find the coefficient $[\tau: \sigma]$ for the 1-dimensional cell $\tau$ which forms the lefthand border of $\sigma$.

Definition 2.1.15 (Acyclic CW-complex). Let $k$ be a field. A CWcomplex $X$ is called acyclic if we have:

$$
H_{i}(X ; k)= \begin{cases}k & \text { for } i=0 \\ 0 & \text { otherwise }\end{cases}
$$

\subsubsection{Simplicial complexes}

CW-complexes form a generalization of spaces called (geometric) simplicial complexes. These are built from affine simplices in the following way:

Definition 2.1.16 (Simplicial complex). $\quad 1$. Let $v_{0}, \ldots, v_{i} \in \mathbb{R}^{d}$ be a finite set of points in Euclidian space. $v_{0}, \ldots, v_{i}$ are called affinely independent if $\sum_{\nu=0}^{i} \lambda_{\nu} v_{\nu}=0$ and $\sum_{\nu=0}^{i} \lambda_{\nu}=0$ implies all $\lambda_{\nu}=0$. (This is equivalent to the property that the smallest affine subspace of $\mathbb{R}^{d}$ which contains the points $v_{0}, \ldots, v_{i}$ has dimension $i$.)

2. An (affine) simplex $\sigma=\left(v_{0}, \ldots, v_{i}\right)$ is defined as the set of all linear combinations $\sum_{\nu=0}^{i} \lambda_{\nu} v_{\nu}$ such that $\sum_{\nu=0}^{i} \lambda_{\nu}=1$ and all $\lambda_{\nu} \geq 0$, where $v_{0}, \ldots, v_{i}$ is a set of affinely independent points in some $\mathbb{R}^{d}$. The affine simplex $\sigma=\left(v_{0}, \ldots, v_{i}\right)$ is then equal to the convex hull of $v_{0}, \ldots, v_{i}$.

3. For a simplex $\sigma=\left(v_{0}, \ldots, v_{i}\right)$ we refer to $i$ as the dimension of $\sigma$.

4. We regard ()$=\emptyset$ as the unique simplex of dimension -1 .

5. Note that if $\sigma=\left(v_{0}, \ldots, v_{i}\right)$ is an affine simplex then $\left(v_{\nu_{0}}, \ldots, v_{\nu_{k}}\right)$ is an affine simplex for any $0 \leq \nu_{0}<\ldots<\nu_{k} \leq i$ as well. We call $\left(v_{\nu_{0}}, \ldots, v_{\nu_{k}}\right)$ a $k$-face of $\sigma$.

6. A set $\Sigma$ of affine simplices is called simplicial complex if

(a) $\sigma \in \Sigma$ and $\tau$ a face of $\sigma$ implies that $\tau \in \Sigma$,

(b) $\sigma \in \Sigma$ and $\tau \in \Sigma$ implies that $\sigma \cap \tau$ is a face of both $\sigma$ and $\tau$.

7. Simplices of $\Sigma$ that are inclusionwise maximal are called facets of $\Sigma$. 
Remark 2.1.17. An abstract simplicial complex is a set $\Sigma \subset \mathcal{P}(V)$ of subsets of a given set $V$ such that for all $A \in \Sigma$ and all $B \subset A$ we have $B \in \Sigma$. In the theory of combinatorial algebraic topology the following facts (see [17]) are well known:

1. For every abstract simplicial complex $\Sigma$ there exists a geometric simplicial complex $\Sigma^{\prime}$ and an isomorphism

$$
\Phi: \Sigma \stackrel{\cong}{\longrightarrow} \Sigma^{\prime}
$$

of partially ordered sets, that is, for all $A, B \in \Sigma$ such that $A \subset B$ we have $\Phi(A) \subset \Phi(B)$. In this situation we say $\Sigma^{\prime}$ is a geometric realisation of $\Sigma$.

2. Two geometric simplicial complexes are linearily isomorphic if and only if they are isomorphic as partially ordered sets.

In the remainder of this thesis we will not distinguish between abstract and geometric simplicial complexes.

To see that indeed simplicial complexes can be viewed as a special class of CW-complexes consider the following

Proposition 2.1.18. (cp. [8], p.245-247) Let $\Sigma$ be a simplicial complex. Then there exist characteristic maps $f_{\sigma}: B^{i} \longrightarrow X:=\bigcup_{\sigma \in \Sigma} \sigma$ that turn $X$ into a $C W$-complex. The closed cells of this $C W$-complex are the affine simplices of $\Sigma$. One can choose the characteristic maps $f_{\sigma}$ such that the coefficients $[\tau: \sigma]$ of the cellular homology of $X$ are given in the following way: If $\sigma=\left(v_{0}, \ldots, v_{i}\right), \tau=\left(v_{0}, \ldots, v_{\nu-1}, v_{\nu+1}, \ldots, v_{i}\right)$ then $[\tau: \sigma]=(-1)^{i}$.

\subsubsection{Graded CW-complexes}

Definition 2.1.19 (Graded CW-complex). 1. For a (not necessarily finite) partially ordered set $(P, \preceq)$ and a map $f:(X, A)^{(*)} \rightarrow P$ we call $(X, A, f)$ a $P$-graded $\mathrm{CW}$-pair if $f$ is order preserving. (See Definition 2.1.9 for the definition of the partial order on $(X, A)^{(*)}$.)

2. If $(P, \preceq)$ is a partially ordered set, $(X, f)$ a $P$-graded CW-complex and $p \in P$, we denote by $X_{\preceq p}$ the $P$-graded sub-CW-complex of $X$ consisting of all cells $\sigma \in X^{(*)}$ such that $f(\sigma) \preceq p$. 


\subsubsection{Cellular chain complexes and cellular resolutions}

$\mathbb{Z}^{d}$-graded free chain complexes and in particular free resolutions can be constructed from graded CW-complexes in the following way:

Definition 2.1.20 (Cellular chain complex, cellular resolution). Let $R$ be a ring. We call a $\mathbb{Z}^{d}$-graded free chain complex

$$
\mathcal{C}=\ldots \stackrel{\partial_{i+1}}{\longrightarrow} \mathcal{C}_{i} \stackrel{\partial_{i}}{\longrightarrow} \mathcal{C}_{i-1} \stackrel{\partial_{i-1}}{\longrightarrow} \ldots \stackrel{\partial_{1}}{\longrightarrow} \mathcal{C}_{0}, \mathcal{C}_{i}=\bigoplus_{\alpha \in \mathbb{Z}^{d}} C_{i}^{\alpha}, C_{i}^{\alpha}=R(-\alpha)^{\beta_{i}^{\alpha}}
$$

cellular if there is a $\left(\mathbb{Z}^{d}, \Lambda\right)$-graded $\mathrm{CW}$-pair $(X, A$, gr $)$ such that:

(a) For all $i \in \mathbb{N}$ there is a basis $e_{\sigma}$ of $C_{i}^{\alpha}$ indexed by the $i$-cells $\sigma$ in $(X, A)^{(i)}$ such that $\operatorname{gr}(\sigma)=\alpha$,

(b) for all $i \in \mathbb{N}-\{0\}, \sigma \in(X, A)^{(i)}$ we have

$$
\partial_{i} e_{\sigma}=\sum_{\sigma \geq \sigma^{\prime} \in X^{(i-1)}}\left[\sigma^{\prime}: \sigma\right] \underline{\mathbf{x}}^{\operatorname{gr}(\sigma)-\operatorname{gr}\left(\sigma^{\prime}\right)} e_{\sigma^{\prime}}
$$

where $\left[\sigma^{\prime}: \sigma\right]$ is the coefficient of $\sigma^{\prime}$ in the differential of $\sigma$ in the cellular chain complex of the pair $(X, A)$.

In this situation we write $\mathcal{C}_{(X, A)}^{\mathrm{gr}}$ for the given chain complex and say that $\mathcal{C}_{(X, A)}^{\mathrm{gr}}$ is supported by the $\left(\mathbb{Z}^{d}, \Lambda\right)$-graded CW-pair $(X, A, \mathrm{gr})$. When the $\mathbb{Z}^{d}$-graded free chain complex $\mathcal{C}$ is a $\mathbb{Z}^{d}$-graded free resolution $\mathcal{C}=\mathcal{F}$, we say that $\mathcal{F}=\mathcal{F}_{(X, A)}^{\text {gr }}$ is a $\mathbb{Z}^{d}$-graded free cellular resolution supported by the $\left(\mathbb{Z}^{d}, \Lambda\right)$-graded CW-pair $(X, A$, gr $)$.

Example 2.1.21. Consider the ideal $M=\left(x_{1}^{2} x_{2}, x_{2}^{2} x_{3}, x_{1} x_{3}^{2}, x_{1} x_{2} x_{3}\right)$ in $S=$ $k\left[x_{1}, x_{2}, x_{3}\right]$. The multigraded minimal free resolution of $M$ over $S$ is given by: 




$$
\begin{aligned}
& S(-(1,1,2)) \\
& \oplus \\
& S(-(1,1,1))
\end{aligned}
$$

This resolution is easily seen to be cellular with $\mathcal{F}=\mathcal{F}_{X}^{\text {gr }}$ for the following graded complex $(X, \mathrm{gr})$ :

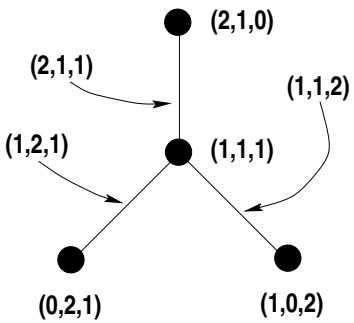

Figure 2.

\subsubsection{Co-Artinian monomial modules}

Definition 2.1.22. $\quad$ 1. We denote by

$$
T:=k\left[x_{1}^{ \pm 1}, \ldots, x_{d}^{ \pm 1}\right]=\bigoplus_{\alpha \in \mathbb{Z}^{d}} k \underline{\mathbf{x}}^{\alpha}
$$

the ring of Laurent polynomials generated by all monomials $\underline{\mathbf{x}}^{\alpha}=x_{1}^{\alpha_{1}}$. $\ldots \cdot x_{d}^{\alpha_{d}}$ for $\alpha=\left(\alpha_{1}, \ldots, \alpha_{d}\right) \in \mathbb{Z}^{d}$.

2. An $S=k\left[x_{1}, \ldots, x_{d}\right]$-module $M$ is called monomial module if it is a submodule of $T$ generated by monomials $\underline{\mathbf{x}}^{\alpha}$ for $\alpha \in \mathbb{Z}^{d}$.

3. A monomial module $M$ is called co-Artinian if for each $\underline{\mathbf{x}}^{\alpha} \in M$ there are only finitely many $\underline{\mathbf{x}}^{\beta} \in M$ such that $\underline{\mathbf{x}}^{\alpha-\beta} \in S$. 
4. Let $M$ be a monomial module. A monomial $\underline{\mathbf{x}}^{\alpha} \in M$ is called minimal if $\frac{\mathbf{x}^{\alpha}}{x_{i}} \notin M$ for all $i=1, \ldots, d$. We denote by $\operatorname{MinGen}(M)$ the set of minimal monomials in $M$.

Lemma 2.1.23. ([5]) A monomial module $M$ is co-Artinian if and only if it is generated by the set $\operatorname{MinGen}(M)$ of its minimal elements.

An ideal in $S=k\left[x_{1}, \ldots, x_{d}\right]$ that is generated by monomials is called a monomial ideal. Of course, any monomial ideal is an example of a co-Artinian monomial module.

Definition 2.1.24. A subgroup $L \subset \mathbb{Z}^{d}$ of the additive group $\mathbb{Z}^{d}$ is called integer lattice.

The following obvious Lemma provides another class of examples of coArtinian monomial modules:

Lemma 2.1.25. Let $L \subset \mathbb{Z}^{d}$ be an integer lattice. Let $M \subset T$ be the monomial module generated by all monomials $\underline{\mathbf{x}}^{\alpha}$ such that $\alpha \in L$. Then $M$ is co-Artinian if and only if $L \cap \mathbb{N}^{d}=\{(0, \ldots, 0)\}$.

\subsection{Some basic facts about cellular resolu- tions}

Definition 2.2.1. Let

$$
\mathcal{C}=\ldots \stackrel{\partial_{i+1}}{\longrightarrow} \mathcal{C}_{i} \stackrel{\partial_{i}}{\longrightarrow} \mathcal{C}_{i-1} \stackrel{\partial_{i-1}}{\longrightarrow} \ldots \stackrel{\partial_{1}}{\longrightarrow} \mathcal{C}_{0}
$$

$\mathcal{C}_{i}=\bigoplus_{\alpha \in \mathbb{Z}^{d}} C_{i}^{\alpha}, C_{i}^{\alpha}=R(-\alpha)^{\beta_{i}^{\alpha}}$ be a $\mathbb{Z}^{d}$-graded free chain complex over the affine semigroup ring $R=k[\Lambda]$. Recall that $\Lambda$ induces a partial order on $\mathbb{Z}^{d}$ by $\alpha \preceq \beta$ if and only if $\alpha+\gamma=\beta$ for some $\gamma \in \Lambda$.

1. We denote by $\mathcal{C} \preceq \alpha\left(\mathcal{C}^{\prec \alpha}\right.$ respectively) the subsequence of $\mathcal{C}$ consisting of the submodules $\mathcal{C}_{i}^{\prec \alpha}:=\bigoplus_{\beta \preceq \alpha} \mathcal{C}_{i}^{\beta}\left(\mathcal{C}_{i}^{\prec \alpha}:=\bigoplus_{\beta \prec \alpha} \mathcal{C}_{i}^{\beta}\right.$ respectively). These are again $\mathbb{Z}^{d}$-graded free chain complexes. They are the subchain complexes of $\mathcal{C}$ generated by all generators with multidegree $\preceq \alpha$ (respectively $\prec \alpha$ ). 
2. Consider the decomposition $\mathcal{C}_{i}=\bigoplus_{\alpha \in \mathbb{Z}^{d}}\left(\mathcal{C}_{i}\right)_{\alpha}$ of $\mathcal{C}_{i}$ as a $k$-module. That is, for all $\alpha \in \mathbb{Z}^{d},\left(\mathcal{C}_{i}\right)_{\alpha}$ consists of all elements of $C_{i}$ of multidegree $\alpha$. By $\mathcal{C}_{\alpha}$ we denote the $k$-chain complex of $k$-vectorspaces given by the degree $\alpha \in \mathbb{Z}^{d}$ components $\left(\mathcal{C}_{i}\right)_{\alpha}$. We call $\mathcal{C}_{\alpha}$ the degree $\alpha$ strand of $\mathcal{C}$.

Note that $\left(\mathcal{C}_{i}\right)_{\alpha} \neq C_{i}^{\alpha}$. In general neither one is a subset of the other.

Remark 2.2.2. Since the differentials $\partial_{i}$ in Definition 2.2 .1 are homogeneous and $R$-linear, they restrict to differentials of $\mathcal{C}^{\prec \alpha}, \mathcal{C} \preceq \alpha$ and $\mathcal{C}_{\alpha}$, so the above chain complexes are well-defined.

\subsubsection{A criterion for a CW-complex to support a cel- lular resolution}

It is helpful to establish the following criterion for a $\left(\mathbb{Z}^{d}, \Lambda\right)$-graded pair $(X, A, g r)$ to support a cellular resolution. The following Proposition was first formulated in [5].

Proposition 2.2.3. Let $R=k[\Lambda]$ be an affine semigroup ring.

1. The $\left(\mathbb{Z}^{d}, \Lambda\right)$-graded $C W$-pair $(X, A$, gr) supports a cellular resolution of a $\mathbb{Z}^{d}$-graded $R$-module if and only if for all $\alpha \in \mathbb{Z}^{d}$ we have that $H_{i}\left(X_{\preceq \alpha}, A_{\preceq \alpha} ; k\right)=0$ for all $i \geq 1$.

2. The $\left(\mathbb{Z}^{d}, \Lambda\right)$-graded $C W$-complex $(X$, gr $)$ supports a cellular resolution of a monomial $R$-module if and only if for all $\alpha \in \mathbb{Z}^{d}$ the subcomplex $X_{\preceq \alpha}$ is acyclic over $k$ or empty.

3. A cellular resolution supported by a $\left(\mathbb{Z}^{d}, \Lambda\right)$-graded pair $(X, A$, gr $)$ is minimal if and only if for all cells $\sigma, \sigma^{\prime} \in(X, A)^{(*)}, \sigma^{\prime} \leq \sigma$ and $\operatorname{dim} \sigma^{\prime}=$ $\operatorname{dim} \sigma-1$ we have either $\operatorname{gr}(\sigma) \neq \operatorname{gr}\left(\sigma^{\prime}\right)$ or $\left[\sigma^{\prime}: \sigma\right]=0$.

Proof. For any multidegree $\alpha$ the degree $\alpha$ strand $\left(\mathcal{F}_{X, A}\right)_{\alpha}$ is supported by basis elements $e_{\sigma}$ for cells $\sigma$ in $\left(X_{\preceq \alpha}, A_{\preceq \alpha}\right)^{(*)}$. Let $z:=\sum_{\sigma \in\left(X_{\preceq \alpha}, A_{\preceq \alpha}\right)^{i}} a_{\sigma} \cdot \underline{\mathbf{x}}^{\alpha-\operatorname{gr} \sigma} \cdot e_{\sigma}$. Then $\partial_{i} z=0$ if and only if the coefficient $b_{\sigma^{\prime}}$ of any $e_{\sigma^{\prime}}, \sigma^{\prime} \in\left(X_{\preceq \alpha}, A_{\preceq \alpha}\right)^{(i-1)}$, 
in $\partial_{i} z$ is zero. Now $b_{\sigma^{\prime}}=\underline{\mathbf{x}}^{\alpha-\operatorname{gr} \sigma^{\prime}} \sum_{\sigma \in\left(X_{\left.{ }_{\alpha}, A_{\preceq \alpha}\right)^{(i)}}\right.} a_{\sigma} \cdot\left[\sigma^{\prime}: \sigma\right]$. Thus $b_{\sigma^{\prime}}=0$ if and only if $\sum_{\sigma \in\left(X_{\preceq \alpha}, A \preceq \alpha\right)} a_{\sigma} \cdot\left[\sigma^{\prime}: \sigma\right]=0$. But this is equivalent to the fact that $\sum_{\sigma \in\left(X_{\preceq \alpha}, A_{\preceq \alpha}\right)^{i}} a_{\sigma} \cdot \sigma$ is a cycle in the cellular chain complex of $\left(X_{\preceq \alpha}, A_{\preceq \alpha}\right)$ with coefficients in $k$.

Similar arguments show that $z:=\sum_{\sigma \in\left(X_{\preceq \alpha}, A_{\preceq \alpha}\right)^{(i)}} a_{\sigma} \cdot \underline{\mathbf{x}}^{\alpha-\operatorname{gr} \sigma} \cdot e_{\sigma}$ is a boundary $z=\partial_{i+1} z^{\prime}$ if and only if $\sum_{\sigma \in\left(X_{\preceq \alpha}, A_{\preceq \alpha}\right)^{(i)}} a_{\sigma} \cdot \sigma$ is a boundary in the cellular chain complex of $\left(X_{\preceq \alpha}, A_{\preceq \alpha}\right)$ with coefficients in $k$.

It follows from Definition 2.1.6 that a resolution $\mathcal{F}_{X, A}$ is minimal if and only if no entry in the matrix describing the differential is a non-zero constant. For cellular resolutions this condition directly translates into the last assertion of the proposition.

\subsubsection{Betti numbers from given resolutions}

By the following lemma the Betti-numbers $\beta_{i}^{\alpha}, \alpha \in \mathbb{Z}^{d}$, of the minimal $\mathbb{Z}^{d}$ graded free resolution of $M$ can be computed from any given $\mathbb{Z}^{d}$-graded free resolution $\mathcal{F}$ of $M$ :

Lemma 2.2.4. Let $M$ be a $\mathbb{Z}^{d}$-graded $R$-module, $\mathcal{F}$ a $\mathbb{Z}^{d}$-graded free resolution of $M$. Then:

$$
\beta_{i}^{\alpha}(M)=\operatorname{dim} H_{i}\left(\left(\mathcal{F}^{\prec \alpha}\right)_{\alpha},\left(\mathcal{F}^{\prec \alpha}\right)_{\alpha}\right) .
$$

Proof. If $\mathcal{F}=\mathcal{F}_{\text {min }}$ is minimal, the assertion follows from the fact that all differentials of the chain complex $\left(\left(\mathcal{F}^{\prec \alpha}\right)_{\alpha},\left(\mathcal{F}^{\prec \alpha}\right)_{\alpha}\right)$ vanish. For general $\mathcal{F}$ there is a direct sum decomposition $\mathcal{F}=\mathcal{F}_{\text {min }} \oplus \mathcal{R}$, where $\mathcal{R}$ is a direct sum of exact sequences

$$
\mathcal{R}_{\alpha, \gamma, i}: \cdots \rightarrow 0 \rightarrow S(-\alpha)^{\gamma} \rightarrow S(-\alpha)^{\gamma} \overbrace{\rightarrow 0}^{i \text { times }} \rightarrow 0
$$


$\alpha \in \mathbb{Z}^{d}, i, \gamma \in \mathbb{N}$. This gives:

$$
\begin{array}{r}
H_{i}\left(\left(\mathcal{F}^{\prec \alpha}\right)_{\alpha},\left(\mathcal{F}^{\prec \alpha}\right)_{\alpha}\right) \cong H_{i}\left(\left(\mathcal{F}_{\text {min }}^{\prec \alpha} \oplus \mathcal{R}^{\preceq \alpha}\right)_{\alpha},\left(\mathcal{F}_{\text {min }}^{\prec \alpha} \oplus \mathcal{R}^{\prec \alpha}\right)_{\alpha}\right) \\
\cong H_{i}\left(\left(\mathcal{F}_{\text {min }}^{\prec \alpha}\right)_{\alpha},\left(\mathcal{F}_{\text {min }}^{\prec \alpha}\right)_{\alpha}\right) \oplus H_{i}\left(\left(\mathcal{R}^{\prec \alpha}\right)_{\alpha},\left(\mathcal{R}^{\prec \alpha}\right)_{\alpha}\right)
\end{array}
$$

and the assertion follows because the sequence $\left((\mathcal{R} \preceq \alpha)_{\alpha},\left(\mathcal{R}^{\prec \alpha}\right)_{\alpha}\right)$ contributes no homology.

Starting with a cellular resolution $\mathcal{F}_{X, A}$ of a $\mathbb{Z}^{d}$-graded $R$-module $M$ and following the idea of Lemma 2.2.4 it is possible to describe the Betti-numbers of the minimal free $\mathbb{Z}^{d}$-graded resolution of $M$ in terms of the homology of the underlying $\left(\mathbb{Z}^{d}, \Lambda\right)$-graded $\mathrm{CW}$-pair $\left(X, A\right.$, gr). We write $H_{i}(X, A ; k)$ for the $i$-th cellular homology group of the pair $(X, A)$ with coefficients in $k$ and $\widetilde{H}_{i}(X ; k)$ for the reduced homology of $X$ with coefficients in $k$. As usual $H_{i}(\emptyset ; k)=0$ for $i \neq-1$ and $H_{-1}(\emptyset ; k)=k$.

Proposition 2.2.5. Let $R=k[\Lambda]$ be an affine semigroup ring. Let $M$ be $a \mathbb{Z}^{d}$-graded $R$-module and $(X, A, f)$ a $\mathbb{Z}^{d}$-graded $C W$-pair supporting a $\mathbb{Z}^{d_{\text {- }}}$ graded free resolution of $M$. Then:

$$
\begin{aligned}
\beta_{i}^{\alpha}(M) & =\operatorname{dim} H_{i}\left(X_{\preceq \alpha}, X_{\prec \alpha} \cup A_{\preceq \alpha} ; k\right) \\
& =\left\{\begin{array}{ll}
\operatorname{dim} H_{i-1}\left(X_{\prec \alpha}, A_{\prec \alpha} ; k\right) & \text { if } A_{\preceq \alpha} \neq \emptyset \\
\operatorname{dim} \tilde{H}_{i-1}\left(X_{\prec \alpha} ; k\right) & \text { if } A_{\preceq \alpha}=\emptyset
\end{array},\right.
\end{aligned}
$$

where the first equation holds for all $i \geq 0$, the second for all $i \geq 2$. If in addition $X_{\preceq \alpha}$ and $A_{\preceq \alpha}$ are acyclic or empty, the second equation also holds for $i=1$. Note that for $i=0$ the righthand side of the first equation is easy to calculate.

In particular, $\beta_{i}^{\alpha}=0$ in case there is no $i$-cell in $(X, A)^{(i)}$ of degree $\alpha$.

Proof. Denoting the $i$-th cellular chain group of a CW-pair by $C_{i}(X, A)$, the chain-complex $\left(\left(\mathcal{F}_{\bar{X}}^{\prec \alpha}, A\right)_{\alpha},\left(\mathcal{F}_{X, A}^{\prec \alpha}\right)_{\alpha}\right)$ reads as follows:

$$
\ldots \rightarrow \frac{C_{i+1}\left(X_{\preceq \alpha}, A_{\preceq \alpha}\right)}{C_{i+1}\left(X_{\prec \alpha}, A_{\prec \alpha}\right)} \rightarrow \frac{C_{i}\left(X_{\preceq \alpha}, A_{\preceq \alpha}\right)}{C_{i}\left(X_{\prec \alpha}, A_{\prec \alpha}\right)} \rightarrow \frac{C_{i-1}\left(X_{\preceq \alpha}, A_{\preceq \alpha}\right)}{C_{i-1}\left(X_{\prec \alpha}, A_{\prec \alpha}\right)} \rightarrow \ldots
$$


with induced differentials. Since we have:

$$
\begin{gathered}
\frac{C_{i}\left(X_{\preceq \alpha}, A_{\preceq \alpha}\right)}{C_{i}\left(X_{\prec \alpha}, A_{\prec \alpha}\right)} \cong \frac{C_{i}\left(X_{\preceq \alpha}\right) / C_{i}\left(A_{\preceq \alpha}\right)}{C_{i}\left(X_{\prec \alpha}\right) / C_{i}\left(A_{\prec \alpha}\right)} \cong \\
\cong \frac{C_{i}\left(X_{\preceq \alpha}\right)}{C_{i}\left(X_{\prec \alpha}\right)+C_{i}\left(A_{\preceq \alpha}\right)} \cong \frac{C_{i}\left(X_{\preceq \alpha}\right)}{C_{i}\left(X_{\prec \alpha} \cup A_{\preceq \alpha}\right)} \cong C_{i}\left(X_{\preceq \alpha}, X_{\prec \alpha} \cup A_{\preceq \alpha}\right)
\end{gathered}
$$

and the induced differentials are the same as those of the cellular chain complex of the pair $\left(X_{\preceq \alpha}, X_{\prec \alpha} \cup A_{\preceq \alpha}\right.$ ), applying Lemma 2.2 .4 proves the first equation.

Applying equation (2.2.2.1), the long exact sequence of the pair $\left(C \bullet\left(X_{\prec \alpha}, A_{\prec \alpha}\right), C_{\bullet}\left(X_{\preceq \alpha}, A_{\preceq \alpha}\right)\right)$ can be written as follows:

$$
\begin{aligned}
\ldots \rightarrow H_{i+1}\left(X_{\preceq \alpha}, A_{\preceq \alpha}\right) & \rightarrow H_{i+1}\left(X_{\preceq \alpha}, X_{\prec \alpha} \cup A_{\preceq \alpha}\right) \longrightarrow \\
\rightarrow H_{i}\left(X_{\prec \alpha}, A_{\prec \alpha}\right) & \rightarrow H_{i}\left(X_{\preceq \alpha}, A_{\preceq \alpha}\right) \rightarrow \ldots
\end{aligned}
$$

This proves the second equation for $i \geq 2$.

Assume now $X_{\preceq \alpha}$ and $A_{\preceq \alpha}$ to be acyclic or empty. The long exact sequence for pairs yields the exact sequence:

$$
\begin{aligned}
0=\widetilde{H}_{1}\left(X_{\preceq \alpha} ; k\right) & \rightarrow \widetilde{H}_{1}\left(X_{\preceq \alpha}, X_{\prec \alpha} \cup A_{\preceq \alpha} ; k\right) \rightarrow \\
\rightarrow \widetilde{H}_{0}\left(X_{\prec \alpha} \cup A_{\preceq \alpha} ; k\right) & \rightarrow \widetilde{H}_{0}\left(X_{\preceq \alpha} ; k\right)=0
\end{aligned}
$$

If $A_{\preceq \alpha}=\emptyset$, we have $\widetilde{H}_{1}\left(X_{\preceq \alpha}, X_{\prec \alpha} \cup A_{\preceq \alpha} ; k\right)=\widetilde{H}_{0}\left(X_{\prec \alpha} ; k\right)$ as stated.

If $A_{\preceq \alpha}$ is acyclic, considering that $X_{\prec \alpha} \cap A_{\preceq \alpha}=A_{\prec \alpha}$, the Mayer-Vietorissequence for $X_{\prec \alpha} \cup A_{\prec \alpha}$ yields $\widetilde{H}_{0}\left(A_{\prec \alpha} ; k\right) \rightarrow \widetilde{H}_{0}\left(X_{\prec \alpha} ; k\right) \rightarrow \widetilde{H}_{0}\left(X_{\prec \alpha} \cup\right.$ $\left.A_{\preceq \alpha} ; k\right) \rightarrow \widetilde{H}_{-1}\left(A_{\prec \alpha} ; k\right) \rightarrow \widetilde{H}_{-1}\left(X_{\prec \alpha} ; k\right)$, while the long exact sequence for the pair $\left(A_{\prec \alpha}, X_{\prec \alpha}\right)$ yields $\widetilde{H}_{0}\left(A_{\prec \alpha} ; k\right) \rightarrow \widetilde{H}_{0}\left(X_{\prec \alpha} ; k\right) \rightarrow H_{0}\left(X_{\prec \alpha}, A_{\prec \alpha} ; k\right) \rightarrow$ $\widetilde{H}_{-1}\left(A_{\prec \alpha} ; k\right) \rightarrow \widetilde{H}_{-1}\left(X_{\prec \alpha} ; k\right)$. The assertion follows from the five lemma.

If there is no $i$-cell of degree $\alpha$ in $(X, A)^{(i)}$ then $H_{i}\left(X_{\preceq \alpha}, X_{\prec \alpha} \cup A_{\preceq \alpha} ; k\right)=$ $H_{i}\left(X_{\preceq \alpha}, X_{\preceq \alpha} ; k\right)=0$. This proves that $\beta_{i}^{\alpha}=0$ in case there is no $i$-cell of degree $\alpha$ in $(X, A)^{(i)}$. 


\subsubsection{Quotients}

In a situation where we have a $\mathbb{Z}^{d}$-graded free resolution $\mathcal{F}$ of a $\mathbb{Z}^{d}$-graded module $M$ and an exact subsequence $\mathcal{G} \hookrightarrow \mathcal{F}$ resolving the submodule $N \hookrightarrow$ $M$, passing to quotients yields a $\mathbb{Z}^{d}$-graded free resolution of the quotient $M / N$ if and only if the modules $\mathcal{G}_{i}$ are direct summands of $\mathcal{F}_{i}$ for all $i \geq 0$. The following cellular situation fullfills this condition:

Proposition 2.2.6. Let the $C W$-pair $(Y, A)$ support a $\mathbb{Z}^{d}$-graded cellular free resolution of the $\mathbb{Z}^{d}$-graded $S$-module $M$ which restricts to a $\mathbb{Z}^{d}$-graded cellular free resolution of a submodule $N \hookrightarrow M$ supported by the $C W$-subpair $(X, A)$ of $(Y, A)$. Passing to quotients yields a $\mathbb{Z}^{d}$-graded cellular free resolution of $M / N$ supported on the $C W$-pair $(Y, X)$.

\subsection{Examples of cellular resolutions}

In this section we present several classes of cellular resolutions. The examples in Sections 2.3.1 - 2.3.5 and 2.3.8 have been known for a while. The examples in Sections 2.3.5 and 2.3.6 are new contributions by the author.

\subsubsection{Taylor resolution}

Recall that for a co-Artinian monomial module $M$ we denote by $\operatorname{MinGen}(M)$ its uniquely defined minimal set of monomial generators (See Definition 2.1.22).

Definition 2.3.1 (Taylor-complex). 1. For a co-Artinian monomial module $M$ let $\mathfrak{T}(M)$ be the simplicial complex on the ground set $\operatorname{MinGen}(M)$ and simplices the finite subsets of $\operatorname{MinGen}(M)$. We grade the simplices $\sigma \in \mathfrak{T}(M)$ by $\operatorname{lcm}(\sigma):=\operatorname{lcm}\{m \mid m \in \sigma\}$. Via the correspondence $\alpha \leftrightarrow \underline{\mathbf{x}}^{\alpha}$, we also regard this grading as a $\mathbb{Z}^{d}$-grading. $\mathfrak{T}(M)$ is called the Taylor-complex of $M$.

2. Let more generally $N \subset M$ be two co-Artinian monomial modules. Then we denote by $\mathfrak{T}(M, N)$ the simplicial complex on the ground set $\operatorname{MinGen}(M) \cup \operatorname{MinGen}(N)$ and simplices the finite subsets of 
$\operatorname{MinGen}(M) \cup \operatorname{MinGen}(N)$. Again, we grade the simplices $\sigma \in \mathfrak{T}(M, N)$ by $\operatorname{lcm}(\sigma):=\operatorname{lcm}\{m \mid m \in \sigma\}$.

Lemma 2.3.2. Let $M$ and $N$ be two co-Artinian monomial modules such that $N \subset M$. Then $\mathfrak{T}(M, N)$ and $\mathfrak{T}(M)$ define cellular resolutions of the monomial module $M$.

Proof. By Proposition 2.2.3 it suffices to show that $\mathfrak{T}(M, N)_{\leq \alpha}$ and $\mathfrak{T}(M)_{\leq \alpha}$ are acyclic or empty complexes for all $\alpha \in \mathbb{Z}^{d}$. If $\mathfrak{T}(M, N)_{\leq \alpha} \neq \emptyset$ or $\mathfrak{T}(M)_{\leq \alpha} \neq \emptyset$, then the simplex which contains all points of multidegree $\alpha$ or less is the unique maximal simplex of the complex. Thus $\mathfrak{T}(M, N)_{\leq \alpha}$ or $\mathfrak{T}(M)_{\leq \alpha}$ is contractible and hence acyclic.

Definition 2.3.3 (Taylor-resolution). 1. For a co-Artinian monomial module $M$ the cellular resolution supported by $\mathfrak{T}(M)$ is called the Taylor-resolution of $M$.

2. Let $N \subset M$ be two co-Artinian monomial modules. The cellular resolution supported by $\mathfrak{T}(M, N)$ is called the Taylor-resolution of the pair $(M, N)$.

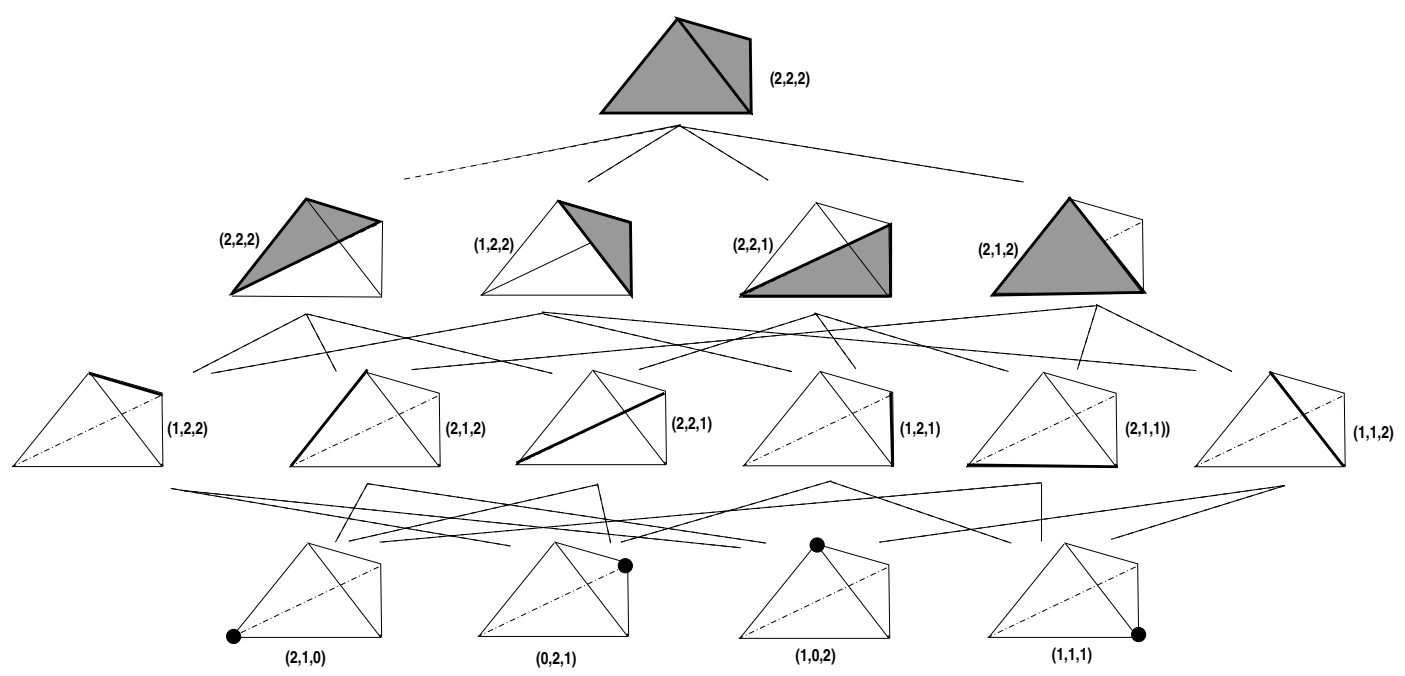

Figure 3. Taylor resolution for Example 2.1.21 
Theorem 2.3.4. Let $N \subset M$ be co-Artinian monomial modules.

Then there exists a cellular $\mathbb{Z}^{d}$-graded free resolution of $M / N$ supported by the pair $(\mathfrak{T}(M, N), \mathfrak{T}(N), \mathrm{lcm})$. In particular, for the Betti-numbers $\beta_{i}^{\alpha}(M / N)$, $\alpha \in \mathbb{Z}^{d}$, of the minimal $\mathbb{Z}^{d}$-graded free resolution of $M / N$ we have for $i \geq 0$ :

$$
\beta_{i}^{\alpha}(M / N)=\left\{\begin{array}{ll}
\operatorname{dim} H_{i-1}\left(\mathfrak{T}(M, N)_{\prec \alpha}, \mathfrak{T}(N)_{\prec \alpha} ; k\right) & \text { if } \mathfrak{T}(N)_{\preceq \alpha} \neq \emptyset \\
\operatorname{dim} \tilde{H}_{i-1}\left(\mathfrak{T}(M, N)_{\prec \alpha} ; k\right) & \text { if } \mathfrak{T}(N)_{\preceq \alpha}=\emptyset
\end{array} .\right.
$$

Proof. Follows immediately from Lemma 2.3.2, Proposition 2.2.6 and Proposition 2.2.5.

Definition 2.3.5. We call the cellular resolution of $M / N$ supported by the pair $(\mathfrak{T}(M, N), \mathfrak{T}(N), \mathrm{lcm})$ the Taylor-resolution of $M / N$.

In order to formulate a condition for a monomial module under which the Taylor-resolution is minimal we need the following definition and obvious remark:

Definition 2.3.6. $\quad 1$. Let $p_{1}, \ldots, p_{n} \in S=k\left[x_{1}, \ldots, x_{d}\right]$ be polynomials. $p_{1}, \ldots, p_{n}$ is called a regular sequence if $\left(p_{1}, \ldots, p_{i-1}\right) \neq S$ and if for all $i=1, \ldots, n$ we have that $p_{i}$ is not a zerodivisor in $S /\left(p_{1}, \ldots, p_{i-1}\right)$. Here, $\left(p_{1}, \ldots, p_{i-1}\right) \subset S$ denotes the ideal generated by the polynomials $p_{1}, \ldots, p_{i-1}$.

2. If $p_{1}, \ldots, p_{n}$ is a regular sequence, the ideal $\left(p_{1}, \ldots, p_{i}\right) \subset S$ is called complete intersection.

Remark 2.3.7. Let $I \subset S$ be a monomial ideal. $I$ is a complete intersection if and only if the elements of $\operatorname{MinGen}(I)$ have disjoint supports, that is, if $m, m^{\prime} \in \operatorname{MinGen}(I), m \neq m^{\prime}$ and $x_{r} \mid m$ for some variable $x_{r}$, then $x_{r} \nmid m^{\prime}$.

Proposition 2.3.8. $\quad$ 1. Let $N \subset M$ be co-Artinian monomial modules. The Taylor-resolution of $M / N$ is minimal if and only if for all $\sigma \in$ $\mathfrak{T}(M, N)$ and all $m \in \sigma$ we have that $m \mid \operatorname{lcm}(\sigma \backslash\{m\})$ implies $\sigma \backslash\{m\} \in$ $\mathfrak{T}(N)$. In particular the Taylor-resolution of $M$ is minimal if and only if for all $\sigma \in \mathfrak{T}(M)$ and all $m \in \sigma$ we have $m \nmid \operatorname{lcm}(\sigma \backslash\{m\})$. 
2. Let $I \subset S=k\left[x_{1}, \ldots, x_{d}\right]$ be a monomial ideal which is a complete intersection. Then the Taylor-resolution of I is minimal.

Proof. Minimality of the Taylor-resolution of $M / N$ occurs if and only if for all simplices $\sigma \in \mathfrak{T}(M, N)$ and all $m \in \sigma$ either the coefficient $\operatorname{lcm} \sigma / \operatorname{lcm}(\sigma \backslash\{m\})$ is an element of the maximal homogeneous ideal $\mathfrak{m}$ or $\sigma \backslash\{m\} \in \mathfrak{T}(N)$. This proves the first part of the Proposition. The second part is an easy application of the first part.

\subsubsection{Scarf resolution}

In search of smaller resolutions Proposition 2.3.8 suggests the following strategy: Start with the Taylor-resolution and remove those simplices that obstruct minimality, namely the simplices $\sigma$ with the following property:

There exists a monomial $m \in \sigma$ such that $m \mid \operatorname{lcm}(\sigma \backslash\{m\})$.

Not very surprisingly minimality is not so easily achieved. The reason is that in general the resulting simplicial complex is not acyclic. Consider the following simple example:

Example 2.3.9. Let $I$ be the ideal in $k\left[x_{1}, x_{2}\right]$ generated by the monomials $x_{1}^{2}, x_{2}^{2}$ and $x_{1} x_{2}$.

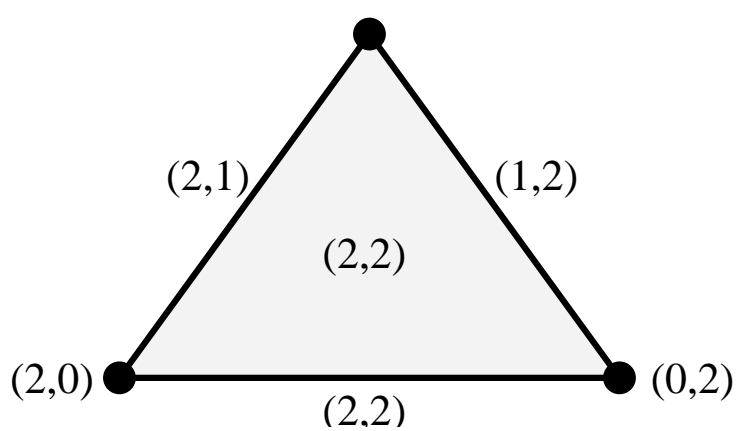

Figure 4. Taylor complex for example 2.3.9

Here, the simplex $\sigma=\left\{x_{1}^{2}, x_{2}^{2}, x_{1} x_{2}\right\}$ has property (2.3.2.1) with $m=$ $x_{1} x_{2}$. Thus, above strategy calls for removing $\sigma$. But since no other simplex 
fullfills property (2.3.2.1) the resulting simplicial complex forms the boundary of a triangle which is not acyclic. In this example this can be fixed by also deleting the simplex $\sigma^{\prime}=\left\{x_{1}^{2}, x_{2}^{2}\right\}$. The result is a minimal resolution.

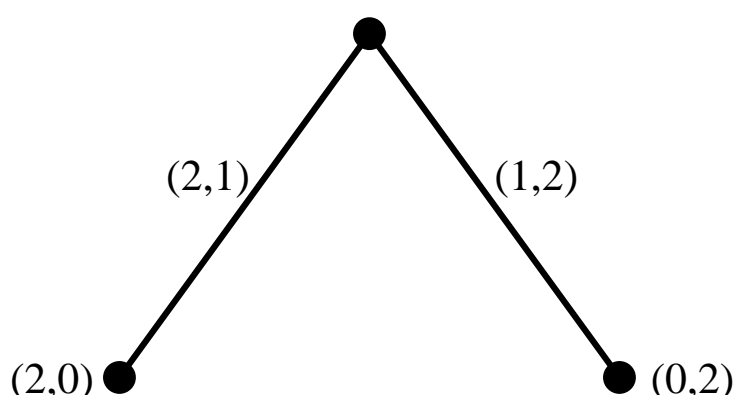

Figure 5. Scarf complex for example 2.3.9

Thus, the next idea is not only to remove the simplices $\sigma=\left\{m_{0}, \ldots, m_{s}\right\}$ with property (2.3.2.1) but also the corresponding simplices $\sigma=\left\{m_{0}, \ldots, m_{s}\right\}$ $-\{m\}$. That is we delete all simplices $\sigma=\left\{m_{0}, \ldots, m_{s}\right\}$ with the property:

There exists $m \in \operatorname{MinGen}(M)$ such that $m \mid \operatorname{lcm}(\sigma \backslash\{m\})$.

The resulting simplicial complex is called the Scarf complex:

Definition 2.3.10 (Scarf-resolution). $\quad$ 1. Let $M \subset T=k\left[x_{1}^{ \pm 1}, \ldots, x_{d}^{ \pm 1}\right]$ be a co-Artinian monomial module. The simplicial subcomplex of the Taylor-complex of $M$ that is given by the set of those simplices $\sigma$ such that there is no other simplex $\tau \neq \sigma$ with $\operatorname{lcm} \sigma=\operatorname{lcm} \tau$ is called the Scarf-complex of $M$.

2. If the Scarf-complex of $M$ is acyclic, we call the resolution supported by it, the Scarf-resolution of $M$.

Unfortunatly, the Scarf-complex in general does not provide a resolution. Consider the next simple example:

Example 2.3.11. Let $I$ be the ideal in $k\left[x_{1}, x_{2}, x_{3}\right]$ generated by the monomials $x_{1} x_{2}, x_{1} x_{3}$ and $x_{2} x_{3}$. 
The resulting Scarf complex consists of three points. Again, this is not an acyclic complex.

Figure 6. Scarf complex for example 2.3.11

There is the following obvious, but noteworthy property of the Scarfresolution:

Proposition 2.3.12. Let $M \subset T=k\left[x_{1}^{ \pm 1}, \ldots, x_{d}^{ \pm 1}\right]$ be a monomial ideal. If the Scarf-resolution of $M$ exists (that is, if the Scarf-complex of $M$ is acyclic), then it is minimal.

Proof. The condition of the second part of Proposition 2.2.3 is obviously satisfied, which proves minimality.

In [5], Bayer and Sturmfels prove existence and therefore minimality of the Scarf-resolution for the class of generic co-Artinian monomial modules. Here is the definition:

Definition 2.3.13. Let $M \subseteq T=k\left[x_{1}^{ \pm 1}, \ldots, x_{d}^{ \pm 1}\right]$ be a co-Artinian monomial module. Let

$$
\partial_{0}: \bigoplus_{m \in \operatorname{MinGen}(M)} S e_{m} \longrightarrow M
$$

be a presentation of $M$, that is, $\partial\left(e_{m}\right)=m$ for all $m \in \operatorname{MinGen}(M)$.

1. A binomial $n_{m} e_{m}-n_{m^{\prime}} e_{m^{\prime}} \in \bigoplus_{m \in \operatorname{MinGen}(M)} S e_{m}$ is called generic if no variable $x_{i}, i=1, \ldots, d$ appears in $m$ and $m^{\prime}$ with the same exponent. 
2. $M$ is called generic if there exists a basis of generic binomials for ker $\partial_{0}$.

Theorem 2.3.14. (see [5], Theorem 2.9) Let $M \subset T=k\left[x_{1}^{ \pm 1}, \ldots, x_{d}^{ \pm 1}\right]$ be a generic co-Artinian monomial module. Then the Scarf-complex supports a cellular minimal free $\mathbb{Z}^{d}$-graded resolution of $M$.

In [4] Bayer, Peeva and Sturmfels prove the same result for a more restrictive version of genericity:

Theorem 2.3.15. (see [4], Theorem 3.2) Let $I \subset S=k\left[x_{1}, \ldots, x_{d}\right]$ be a monomial ideal such that for any $m, m^{\prime} \in \operatorname{Min} \operatorname{Gen}(M), m \neq m^{\prime}$, no variable appears in $m$ and $m^{\prime}$ with the same non-zero exponent. Then the Scarfcomplex supports a cellular minimal free $\mathbb{Z}^{d}$-graded resolution of $I$.

\subsubsection{Hull resolution}

In [5] Bayer and Sturmfels introduce another cellular resolution called the Hull-resolution.

Definition 2.3.16. 1 . For a co-Artinian monomial module the Hullresolution is the cellular resolution supported on the polytopal $\mathbb{Z}^{d}$ graded subcomplex $X_{\text {Hull }}$ of $\mathbb{R}^{d}$ whose faces are the bounded faces of the polyhedral complex which is the convex hull of $\left(t^{\alpha_{1}}, \ldots, t^{\alpha_{d}}\right) \in \mathbb{R}^{d}$ for $\underline{\mathbf{x}}^{\alpha} \in M$ and $t$ sufficiently large. (See [5, Theorem 2.3] for details.)

2. For a face $c$ of $X_{\text {Hull }}$ its degree $\operatorname{lcm}(c)$ is given by $\alpha$ such that $\underline{\mathbf{x}}^{\alpha}$ is the lcm of the monomials in $M$ corresponding to the vertices of the face $c$.

Since by [5, Example 3.11] the Hull complex is in general not a locally finite polytopal complex, its topology differs from the one induced by the topology of $\mathbb{R}^{d}$, see [8, Theorem 8.2]. But it is locally finite in the important case of co-Artinian monomial modules defined by lattices [5, Theorem 3.14].

The Hull-resolution can be viewed as a generalization of the Scarfresolution, since it always exists and there is the following Theorem:

Theorem 2.3.17. (See [5], Theorem 2.9) If $M \subset T=k\left[x_{1}^{ \pm 1}, \ldots, x_{d}^{ \pm 1}\right]$ is generic, the Hull-resolution coincides with the Scarf-resolution and is therefore minimal. 


\subsubsection{Lyubeznik's resolution}

There is another way to continue the approach discussed in Section 2.3.2. Starting from the Taylor-resolution we discussed the two strategies of either deleting all simplices with property (2.3.2.1) or even deleting all simplices with property (2.3.2.2). What we found in Examples 2.3.9 and 2.3.11 suggests to do "something in between": In Example 2.3.9, deleting all simplices $\sigma$ with property (2.3.2.1) was not enough. In Example 2.3.11 deleting even all simplices with property (2.3.2.2) was too much.

Lyubeznik presents in [16] a nice procedure to delete some simplices with property 2.3.11 from the Taylor-resolution according to a given linear order on $\operatorname{Min} \operatorname{Gen}(M)$ which he shows always results in an acyclic subcomplex of the Tylor-complex and therefore gives rise to a cellular free resolution of $M$ :

Definition 2.3.18 (Lyubeznik-resolution). $\quad$ 1. Let $\preceq$ be a linear ordering of MinGen $(M)$. Then the Lybeznik complex is defined to be the simplicial complex on the ground set $\operatorname{MinGen}(M)$ that consists of all simplices $\sigma=\left\{m_{0}, \ldots, m_{s}\right\}, m_{0} \prec m_{1} \prec \ldots \prec m_{s}$ that satisfy:

$$
\begin{gathered}
\text { for all } t<s \text { and } m \in \operatorname{MinGen}(M) \text { s. t. } m \prec m_{t} \text { we have } \\
\qquad m \nmid \operatorname{lcm}\left\{m_{t}, \ldots, m_{s}\right\} .
\end{gathered}
$$

2. Lyubeznik's resolution is the $\mathbb{Z}^{d}$-graded cellular free resolution supported by the Lyubeznik complex.

Above definition means that one gets the Lyubeznik complex from the Taylor complex by deleting those simplices $\sigma=\left\{m_{0}, \ldots, m_{s}\right\}, m_{0} \prec m_{1} \prec$ $\ldots \prec m_{s}$ with property (2.3.2.2) that contain a "tail" $\left\{m_{t}, \ldots, m_{s}\right\}$ for which there exists a monomial $m \in \operatorname{MinGen}(M)$ such that $m \prec m_{t}$ and $m \mid \operatorname{lcm}\left\{m_{t}, \ldots, m_{s}\right\}$. 


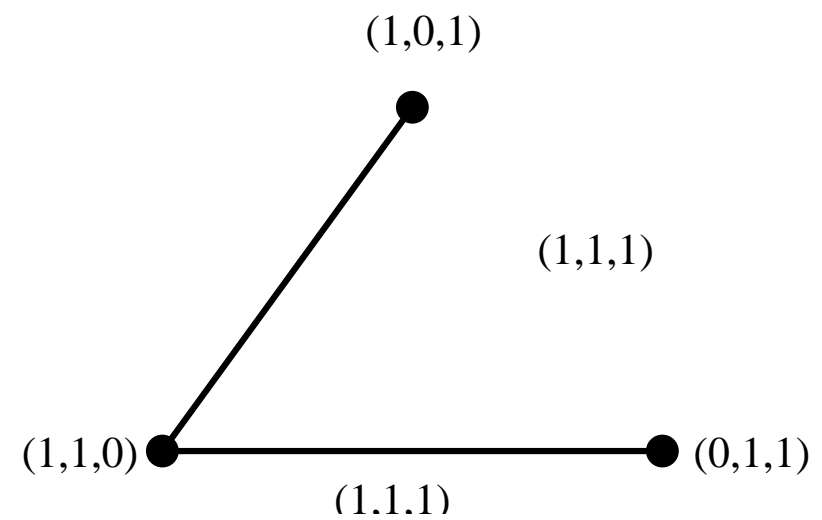

Figure 7. Lyubeznik's complex for example 2.3.11 when using the ordering $x_{1} x_{2} \prec x_{1} x_{3} \prec x_{2} x_{3}$

\subsubsection{Resolutions via rooted complexes}

Definition 2.3.19. A partially ordered $P$ set is called a lattice if for all $x, y \in P$ there exists both

- an element $z \in P$ such that $x, y \preceq z$ and for all $u \in P$ such that $x, y \preceq u$ we have $z \preceq u$, called the join $x \vee y$ of $x$ and $y$, and

- an element $z^{\prime} \in P$ such that $z^{\prime} \preceq x, y$ and for all $u \in P$ such that $u \preceq x, y$ we have $u \preceq z^{\prime}$, called the meet $x \wedge y$ of $x$ and $y$.

For co-Artinian monomial modules we will frequently make use of the following lattice associated to it:

Definition 2.3.20. Let $M \subset k\left[x_{1}^{ \pm 1}, \ldots, x_{d}^{ \pm 1}\right]$ be a co-Artinian monomial module. We define $\mathrm{LCM}(\mathrm{M})$ to be the lattice consisting of all lcm's of finite subsets of $\operatorname{MinGen}(M)$.

Note that $1=\operatorname{lcm}(\emptyset) \in \operatorname{LCM}(M)$ garantees that this really is a lattice. 


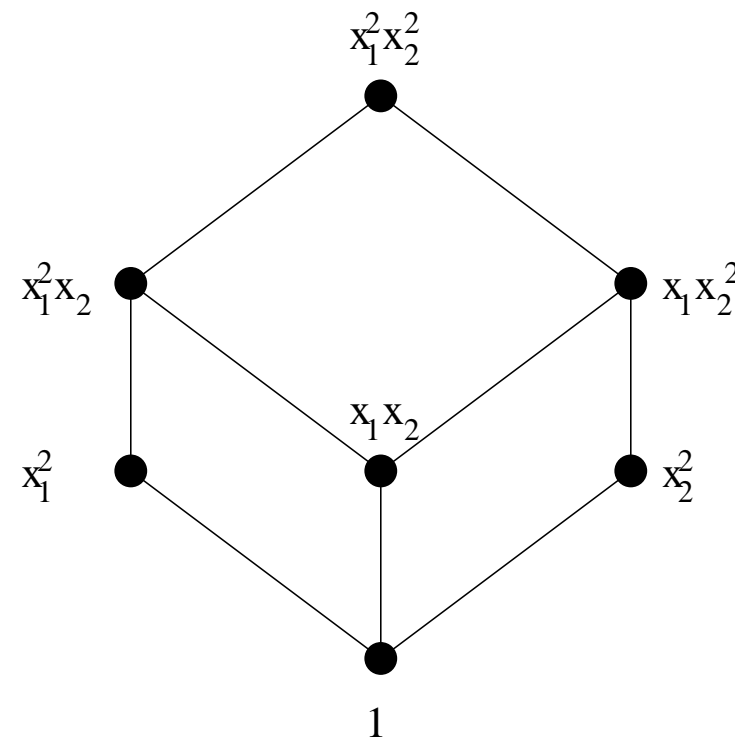

Figure 8. LCM-poset for example 2.3.9

Novik presents in [18] a generalisation to Lyubeznik's resolution which is constructed via rooted complexes. These were introduced by Björner and Ziegler in [7].

Definition 2.3.21. $\quad$ 1. A rooting map for a monomial ideal $I$ is a function

$$
\pi: \operatorname{LCM}(\mathrm{I}) \longrightarrow \operatorname{MinGen}(\mathrm{I})
$$

such that for all $m \in \operatorname{LCM}(\mathrm{I})$ we have

$$
\begin{aligned}
\pi(m) & \mid m \\
\pi(m)\left|m^{\prime}\right| m & \Rightarrow \pi\left(m^{\prime}\right)=\pi(m) \text { for all } m^{\prime} \in \operatorname{LCM}(\mathrm{I})
\end{aligned}
$$

2. Given a rooting map $\pi$ and a nonempty subset $S$ of $\operatorname{MinGen}(I)$, we define

$$
\tilde{\pi}(S):=\pi(\operatorname{lcm}(S))
$$

We say that a subset $S$ of $\operatorname{MinGen}(I)$ is unbroken if $\tilde{\pi}(S) \in S$. We call $S$ rooted if all nonempty subsets of $S$ are unbroken. The rooted subsets of MinGen $(I)$ obviously form a simplicial complex which is called the rooted complex $\mathcal{R} C(I, \pi)$ of $I$ (with respect to the rooting map $\pi$ ). 
Novik proves in [18]:

Theorem 2.3.22. Let $I$ be a monomial ideal, $\pi$ a rooting map for $I$. Then the rooted complex $\mathcal{R} C(I, \pi)$ supports a $\mathbb{Z}^{d}$ graded cellular free resolution of $I$.

This indeed is a generalisation of Lyubeznik's construction: Let $\preceq$ be a linear order on MinGen $(I)$. Define a rooting map by setting

$$
\pi(m):=\min _{\preceq}\left\{m^{\prime} \in \operatorname{MinGen}(I)\left|m^{\prime}\right| m\right\} .
$$

It is easy to see that under this choice of the rooting map $\pi$ the rooted complex $\mathcal{R} C(I, \pi)$ coincides with the Lyubeznik complex.

Novik also presents a condition under which the resolution given by rooted complexes is minimal.

For the statement we need the following definition:

Definition 2.3.23. A finite lattice $L$ is called geometric if it is atomic, that is, every element is a join of atoms, and semimodular, which means that if for elements $x, y \in L$ there exists no element $z \in L$ such that $x \wedge y<z<x$ then there also exists no element $z \in L$ such that $y<z<x \vee y$.

Theorem 2.3.24. Let I be a monomial ideal, such that $\mathrm{LCM}(\mathrm{I})$ is a geometric lattice and let $\pi$ be a rooting map for $I$. Then the rooted complex $\mathcal{R} C(I, \pi)$ supports a cellular free resolution of $I$.

\subsubsection{LCM- and $\mathrm{LCM}^{*}$ - resolutions}

In this section we introduce new cellular resolutions of monomial modules derived from their LCM-lattices. Consider the following example:

Example 2.3.25. Let $M \subset k\left[x_{1}, \ldots, x_{d}\right]$ be the monomial module generated by all $d$ monomials of the form $x_{1} \ldots x_{i-1} x_{i+1} \ldots x_{d}, i=1, \ldots, d$. There is a cellular resolution of $M$ given by a onedimensional simplicial complex consisting of $d+10$-cells and $d 1$-cells. 
Proof. Consider the following $\mathbb{Z}^{d}$-graded simplicial complex $X$ : There are $d+1$ points, the first $d$ of them correspond to the minimal generators of $M$ and are graded accordingly. The last point is graded by $(1, \ldots, 1) \in \mathbb{Z}^{d}$. For each of the first $d$ points there is a line connecting it with the last point. All these lines are graded by $(1, \ldots, 1) \in \mathbb{Z}^{d}$. It is easily seen that this graded simplicial complex satisfies the condition of Proposition 2.2.3 since for all $\alpha \in \mathbb{Z}^{d}$ the subcomplex $X_{\leq \alpha}$ is empty, consists of one point or equals the whole complex $X$.

Although the above resolution has one 0-cell more than the Taylor-resolution and therefore does not qualify for minimality it has much fewer cells of all other dimensions. The above resolution is an example of the following class of resolutions which we will call LCM-resolutions:

Definition 2.3.26. $\quad$ 1. Let $P$ be a partially ordered set. The simplicial complex $\Delta(P)$ that consists of all finite chains in $P$, that is all finite subsets of $P$ that are totally ordered by the partial order of $P$, is called the order complex of $P$.

2. Let $M \subset k\left[x_{1}^{ \pm 1}, \ldots, x_{d}^{ \pm 1}\right]$ be a co-Artinian monomial module. Denote by $X_{\mathrm{LCM}}(M)$ the order complex of $\operatorname{LCM}(\mathrm{M})-\{1\}$ (see Definition 2.3.20 for the definition of $\operatorname{LCM}(\mathrm{M}))$. We refer to this complex as the LCMcomplex of $M$. We grade the simplices of $X_{\mathrm{LCM}}(M)$ in the obvious way by $\mathrm{lcm}$.

Proposition 2.3.27. Let $M$ be a co-Artinian monomial module. Then there is a $\mathbb{Z}^{d}$-graded cellular free resolution of $M$ supported by $X_{\mathrm{LCM}}(M)$.

We call this resolution the LCM-resolution of $M$. 


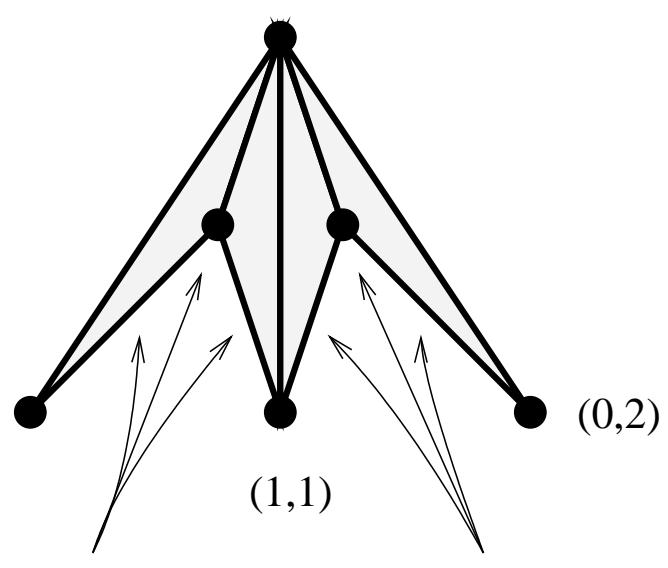

(2.1)

Figure 9. LCM-complex for example 2.3.9

All simplices that contain the point corresponding to the monomial $x_{1}^{2} x_{2}^{2}$ are graded with $(2,2) \in \mathbb{Z}^{2}$.

Proof. For all $\alpha \in \mathbb{Z}^{d}$ there exists a unique 0-chain $\left\{\underline{\mathbf{x}}^{\alpha}\right\}$ graded with $\alpha$. For all simplices $\sigma$ of the subcomplex $\left(X_{\mathrm{LCM}}(M)\right)_{\leq \alpha}$ one of the following is true:

1. $\underline{\mathbf{x}}^{\alpha} \in \sigma$ and $\sigma-\left\{\underline{\mathbf{x}}^{\alpha}\right\} \in\left(X_{\mathrm{LCM}}(M)\right)_{\leq \alpha}$,

2. $\underline{\mathbf{x}}^{\alpha} \notin \sigma$ and $\sigma \cup\left\{\underline{\mathbf{x}}^{\alpha}\right\} \in\left(X_{\mathrm{LCM}}(M)\right)_{\leq \alpha}$.

Hence $\left(X_{\mathrm{LCM}}(M)\right)_{\leq \alpha}$ forms a cone, therefore is contractible and acyclic. The assertion follows with Proposition 2.2.3.

We now introduce another class of cellular resolutions which is similar to the class of LCM-resolutions:

Definition 2.3.28. Let $M \subset k\left[x_{1}^{ \pm 1}, \ldots, x_{d}^{ \pm 1}\right]$ be a co-Artinian monomial module. Let $\mathrm{LCM}^{*}(\mathrm{M})$ be the partially ordered set consisting of all monomials in $M$ that devide lcm $\sigma$ for some finite subset $\sigma \subset \operatorname{MinGen}(M)$. We set $\Delta\left(\mathrm{LCM}^{*}(\mathrm{M})\right)$ to be the order complex of $\mathrm{LCM}^{*}(\mathrm{M})$ graded by $\mathrm{lcm}$ in the obvious way. We refer to this complex as the $\mathrm{LCM}^{*}-$ complex. 
Note that $\mathrm{LCM}^{*}(\mathrm{M})$ is the convex hull of $\operatorname{LCM}(\mathrm{M})-\{1\}$ in the set of all monomials in $k\left[x_{1}^{ \pm 1}, \ldots, x_{d}^{ \pm 1}\right]$ in the sense that we get $\operatorname{LCM}^{*}(\mathrm{M})$ from $\operatorname{LCM}(\mathrm{M})-\{1\}$ by adding all monomials $m \in k\left[x_{1}^{ \pm 1}, \ldots, x_{d}^{ \pm 1}\right]$ with the property that there exist monomials $m_{1}, m_{2} \in \mathrm{LCM}(\mathrm{M})$ such that $m_{1}|m| m_{2}$.

Proposition 2.3.29. Let $M$ be a co-Artinian monomial module. Then there is a $\mathbb{Z}^{d}$-graded cellular free resolution of $M$ supported by $\Delta\left(\mathrm{LCM}^{*}(\mathrm{M})\right)$.

Proof. The proof is the same as for Proposition 2.3.27.

\subsubsection{Hypersimplex-resolution of the maximal homo- geneous ideal}

In this subsection we present a new $\mathbb{Z}^{d}$-graded cellular free resolution of the powers $\mathfrak{m}^{n}$ of the maximal homogeneous ideal $\mathfrak{m}=\left(x_{1}, \ldots, x_{d}\right) \subset S=$ $k\left[x_{1}, \ldots, x_{d}\right]$.

Definition 2.3.30. Let $\mathcal{C}_{d}^{n}$ be the polytopal CW-complex with

$$
\Delta_{n}:=n \cdot \Delta^{d-1}=\left\{\left(y_{1}, \ldots, y_{d}\right) \in \mathbb{R}^{d} \mid \sum_{i=1}^{d} y_{i}=n, y_{i} \geq 0, i=1, \ldots, d\right\}
$$

as underlying space and CW-complex-structure induced by intersection with the cubical CW-complex-structure on $\mathbb{R}^{d}$ given by the integer lattice $\mathbb{Z}^{d}$. That is, the closed cells of $\mathcal{C}_{d}^{n}$ are given by all hypersimplices

$$
\begin{aligned}
C_{\underline{a}, J}:=\Delta_{n} \cap & \left\{\left(y_{1}, \ldots, y_{d}\right) \in \mathbb{R}^{d} \mid y_{i}=a_{i}: i \in[d] \backslash J, y_{j} \in\left[a_{j}, a_{j}+1\right]: j \in J\right\} \\
& =\operatorname{Conv}\left(\underline{a}+\sum_{j \in J} \epsilon_{j} e_{j}\left|\epsilon_{j} \in\{0,1\}, \sum_{j \in J} \epsilon_{j}=d-\right| \underline{a} \mid\right)
\end{aligned}
$$

with $\underline{a} \in \mathbb{N}^{d}, J \subset[d]:=\{1, \ldots, d\},|\underline{a}|:=\sum_{i \in[d]} a_{i}, e_{i}$ the $i$-th unit vector in $\mathbb{R}^{d}$, either subject to the conditions $|\underline{a}|=n$ and $J=\emptyset$, (these are the 0 -cells $C_{\underline{a}, \emptyset}=\{\underline{a}\}$, ) or the condition $1 \leq n-|\underline{a}| \leq|J|-1$. The CW-complex $\mathcal{C}_{d}^{n}$ is naturally multigraded by setting $\operatorname{lcm}\left(C_{\underline{a}, J}\right):=\underline{a}+\sum_{j \in J} e_{j}$. We refer to $\mathcal{C}_{d}^{n}$ as the hypersimplicial complex. 


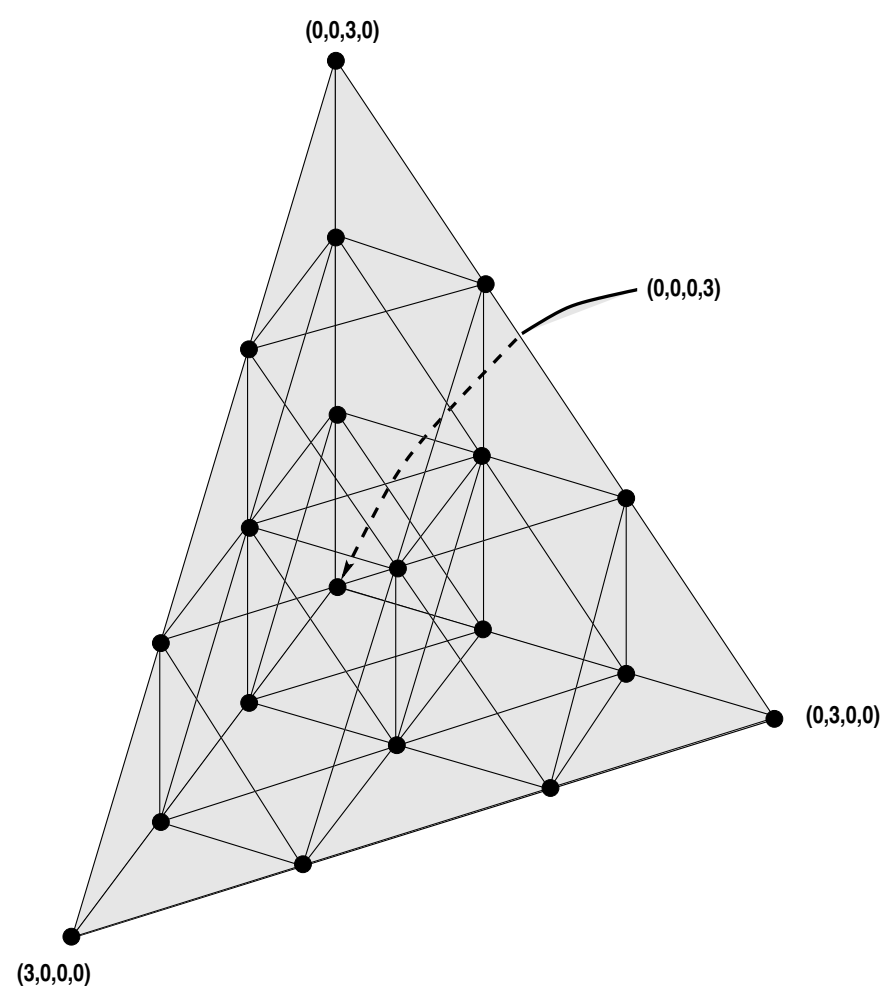

Figure 10. The hypersimplicial complex $\mathcal{C}_{4}^{3}$

Lemma 2.3.31. Considering canonical orientations of these hypersimplices and denoting $J=\left\{j_{0}, \ldots, j_{r}\right\}, j_{0}<\ldots<j_{r}, J_{\nu}:=J \backslash\left\{j_{\nu}\right\}$, the differential of $\mathcal{C}_{d}^{n}$ is given by

- $\partial C_{\underline{a}, J}=\sum_{\nu=0}^{r}(-1)^{\nu}\left(C_{\underline{a}, J_{\nu}}-C_{\underline{a}+e_{j_{\nu}}, J_{\nu}}\right), \quad$ if $\quad 2 \leq n-|\underline{a}| \leq|J|-2$,

$\begin{array}{cc}\text { - } & \partial C_{\underline{a}, J}=\sum_{\nu=0}^{r}(-1)^{\nu} C_{\underline{a}, J_{\nu}}, \quad \text { if } \quad 1=n-|\underline{a}| \leq|J|-2, \\ \text { - } & \partial C_{\underline{a}, J}=\sum_{\nu=0}^{r}(-1)^{\nu+1} C_{\underline{a}+e_{j_{\nu}}, J_{\nu}}, \quad \text { if } \quad 2 \leq n-|\underline{a}|=|J|-1,\end{array}$

- $\partial C_{\underline{a},\left\{j_{0}, j_{1}\right\}}=C_{\underline{a}+e_{j_{1}}, \emptyset}-C_{\underline{a}+e_{j_{0}}, \emptyset}, \quad$ if $\quad n-|\underline{a}|=1$,
$C_{\underline{a}, \emptyset}=0$,
if $\quad|\underline{a}|=n$. 
Proposition 2.3.32. $\mathcal{C}_{d}^{n}$ defines a multigraded cellular free resolution of $\mathfrak{m}^{n}$.

Proof. The 0-cells of $\mathcal{C}_{d}^{n}$ are in one-to-one-correspondence with the set of minimal generators of $\mathfrak{m}^{n}$, so the fact that $\left(\mathcal{C}_{d}^{n}\right)_{\leq \alpha}=\Delta_{n} \cap\left\{\left(y_{1}, \ldots, y_{d}\right) \in\right.$ $\left.\mathbb{R}^{d} \mid y_{i} \leq \alpha_{i}, i=1, \ldots, d\right\}$ is contractible or empty for all $\alpha \in \mathbb{Z}^{d}$ proves (use Proposition 2.2.3) the assertion.

\subsubsection{Bar-resolution}

Definition 2.3.33. Let $k[\Lambda]$ be an affine semigroup ring. We set $\Lambda_{0}:=\Lambda \backslash$ $\{\underline{\mathbf{0}}\}$. The (normalized) Bar-resolution of $k$ as an $R$-module is the multigraded resolution

$$
\mathcal{F}=\ldots \stackrel{\partial_{i+1}}{\longrightarrow} \mathcal{F}_{i} \stackrel{\partial_{i}}{\longrightarrow} \mathcal{F}_{i-1} \stackrel{\partial_{i-1}}{\longrightarrow} \ldots \stackrel{\partial_{1}}{\longrightarrow} \mathcal{F}_{0}
$$

$\mathcal{F}_{i}=\bigoplus_{\alpha \in \mathbb{Z}^{d}} F_{i}^{\alpha}$ such that $F_{i}^{\alpha}$ is the free $R$-module with basis given by the set

$$
\Lambda_{i}^{\alpha}:=\left\{\left[\lambda_{0}|\cdots| \lambda_{i}\right] \mid \lambda_{j} \in \Lambda_{0}, 0 \leq j \leq i, \lambda_{0}+\cdots+\lambda_{i}=\alpha\right\}
$$

The differential is given by

$$
\partial_{i}\left[\lambda_{0}|\cdots| \lambda_{i}\right]=\underline{\mathbf{x}}^{\lambda_{0}}\left[\lambda_{1}|\cdots| \lambda_{i}\right]+\sum_{j=1}^{i}(-1)^{j}\left[\lambda_{0}|\cdots| \lambda_{j-1}+\lambda_{j}|\cdots| \lambda_{i}\right]
$$

To see that this really defines a $\mathbb{Z}^{d}$-graded free resolution of $k$, consider the following Lemma:

Lemma 2.3.34. The Bar-resolution is cellular. It is supported by the $\left(\mathbb{Z}^{d}, \Lambda\right)$ graded order-complex $\Delta\left(\Lambda_{0}\right)$ of $\Lambda_{0}$.

Proof. For $\sigma=\left(\lambda_{0}, \ldots, \lambda_{i}\right) \in \Delta(\Lambda)^{(*)}$, that is $\lambda_{0} \prec \ldots \prec \lambda_{i}$, we set $\operatorname{gr}(\sigma):=\lambda_{i}$. By Definition 2.1.1 it is obvious that this really provides a $\left(\mathbb{Z}^{d}, \Lambda\right)$-grading for the order-complex $\Delta\left(\Lambda_{0}\right)$ of $\Lambda_{0}$. We set $\Delta\left(\Lambda_{0}\right)_{\alpha}^{(i)}:=\{\sigma \in$ $\left.\Delta\left(\Lambda_{0}\right)^{(i)} \mid \operatorname{gr}(\sigma)=\alpha\right\}$. Consider the following maps 


$$
\begin{aligned}
& \Phi_{\alpha}^{i}: \quad \Lambda_{i}^{\alpha} \quad \longrightarrow \Delta\left(\Lambda_{0}\right)_{\alpha}^{(i)} \\
& {\left[\lambda_{0}, \ldots, \lambda_{i}\right] \longmapsto\left(\lambda_{i}, \lambda_{i-1}+\lambda_{i}, \ldots, \lambda_{0}+\ldots+\lambda_{i}\right)}
\end{aligned}
$$

For all $i \in \mathbb{N}$ the map $\Phi_{\alpha}^{i}$ provides a one-to-one correspondences between $\Lambda_{i}^{\alpha}$ and $\Delta\left(\Lambda_{0}\right)_{\alpha}^{(i)}$. The differential of the simplicial complex $\Delta\left(\Lambda_{0}\right)$ induces the following map:

$$
\left[\lambda_{0}|\cdots| \lambda_{i}\right] \longmapsto\left[\lambda_{1}|\cdots| \lambda_{i}\right]+\sum_{j=1}^{i}(-1)^{j}\left[\lambda_{0}|\cdots| \lambda_{j-1}+\lambda_{j}|\cdots| \lambda_{i}\right] .
$$

The given differential comes from homogenising this map. This proves the assertion. 


\section{Chapter 3}

\section{Discrete Morse theory}

In this chapter we discuss discrete Morse theory. This is the fundamental tool from combinatorial topology used in this thesis. Section 3.1 presents parts of Forman's discrete Morse theory for regular CW-complexes. Section $3.2 \mathrm{ex}-$ pands this theory to not necessarily finite graded CW-complexes. In Section 3.3 we develop a discrete Morse theory for cellular resolutions. Section 3.4 discusses Morse differentials.

\subsection{Discrete Morse theory for CW-complexes}

The fundamental tool from combinatorial topology applied in this thesis is discrete Morse theory as developed by Forman [13, 14]. In this section, we review parts of this theory.

Definition 3.1.1. Let $X$ be a CW-complex, $\sigma, \tau \in X^{(*)}$ cells in $X$ such that $\sigma$ is a face of $\tau, \operatorname{dim}(\sigma)=i-1, \operatorname{dim}(\tau)=i$. Let

$$
f_{\sigma}: B^{i-1} \rightarrow X \text { and } f_{\tau}: B^{i} \rightarrow X
$$

be the characteristic maps for $\sigma$ and $\tau$. We say that $\sigma$ is a regular face of $\tau$ if there exist homeomorphisms

$$
g_{\tau}: B^{i} \stackrel{\cong}{\longrightarrow} B^{i}
$$

and

$$
g_{\sigma}: S_{\geq 0}^{i-1}:=\left\{\left(x_{1}, \ldots, x_{i}\right) \in S^{i-1} \mid x_{1} \geq 0\right\} \stackrel{\cong}{\longrightarrow} B^{i-1}
$$


such that the following diagram commutes:



The central idea of discrete Morse theory is to construct for a given CWcomplex a new CW-complex that is homotopically equivalent to the original one but is built from less cells. The condition needed for such a construction is the existence of a discrete Morse function.

Definition 3.1.2 (discrete Morse function). A discrete Morse function on the CW-complex $X$ is a function

$$
f: X^{(*)} \longrightarrow \mathbb{R}
$$

such that

1. for every cell $\sigma \in X^{(*)}$

$$
\begin{aligned}
& \mid\left\{\tau \in X^{(*)} \mid \tau \text { is a facet of } \sigma, f(\tau) \geq f(\sigma)\right\} \mid \leq 1 \\
& \mid\left\{\tau \in X^{(*)} \mid \sigma \text { is a facet of } \tau, f(\tau) \leq f(\sigma)\right\} \mid \leq 1
\end{aligned}
$$

and

2. if $\sigma, \tau \in X^{(*)}$ are cells in $X, \sigma$ a facet of $\tau$, such that

$$
f(\sigma) \geq f(\tau)
$$

then $\sigma$ is a regular facet of $\tau$.

Trivial examples of discrete Morse functions are all functions on $X^{(*)}$ that increase strictly with dimension. Discrete Morse functions can be regarded as functions that increase with dimension up to one exception locally. 
Definition 3.1.3 (critical cells). Let $X$ be a finite CW-complex, $f$ a discrete Morse function on $X$. A cell $\sigma$ of $X$ is called $f$-critical, if

$$
\begin{aligned}
& \mid\left\{\tau \in X^{(*)} \mid \tau \text { is a facet of } \sigma, f(\tau) \geq f(\sigma)\right\} \mid=0 \\
& \mid\left\{\tau \in X^{(*)} \mid \sigma \text { is a facet of } \tau, f(\tau) \leq f(\sigma)\right\} \mid=0 .
\end{aligned}
$$

We set

$$
X_{\text {critical }}^{(*)}(f):=\left\{\sigma \in X^{(*)} \mid \sigma \text { is } f \text {-critical }\right\}
$$

For every dimension $i$ the number of $f$-critical cells in dimension $i$ is called the Morse number $m_{i}(f)$.

As one main result Forman shows (see [13], Theorem 10.2):

Theorem 3.1.4. Let $X$ be a finite $C W$-complex, $f$ a discrete Morse function on $X$. Then $X$ is homotopy equivalent to a $C W$-complex with exactly $m_{i}(f)$ cells of dimension $i$.

\subsection{Discrete Morse theory for graded CW- complexes}

In this section we expand discrete Morse theory to graded and not necessarily finite CW-complexes. In [9], Chari reformulates discrete Morse theory in terms of acyclic matchings. In this section we review his results. On the way we show that his approach leads in a natural way to the consideration of gradings by partially ordered sets. As main result of this section (see Theorem 3.2.14) we prove a version of Theorem 3.1.4 for graded CW-complexes.

\subsubsection{Acyclic matchings}

Definition 3.2.1 (cell graph). Let $X$ be a CW-complex. Consider the directed graph $G_{X}$ on $X^{(*)}$ whose set $E_{X}$ of edges is given by

$$
E_{X}:=\{\tau \rightarrow \sigma \mid \sigma \text { is a facet of } \tau\} .
$$

We call $G_{X}=\left(X^{(*)}, E_{X}\right)$ the cell graph of $X$. 
Definition 3.2.2 (acyclic matching). Let $X$ be a CW-complex, $G_{X}=$ $\left(X^{(*)}, E_{X}\right)$ its cell graph. Let $A \subset E_{X}$ be a subset of edges $\tau \rightarrow \sigma \in E_{X}$, such that $\sigma$ is a regular face of $\tau$ for all $\tau \rightarrow \sigma \in E_{X}$.

1. We denote by $G_{X}^{A}=\left(X^{(*)}, E_{X}^{A}\right)$ the induced graph with edge set

$$
E_{X}^{A}:=\left(E_{X} \backslash A\right) \cup\{\sigma \rightarrow \tau \mid \tau \rightarrow \sigma \in A\}
$$

that is built from $G_{X}$ by reversing the direction of all edges $\tau \rightarrow \sigma$ that lie in $A$.

We call an edge $\sigma \rightarrow \tau \in E_{X}^{A}$ an $A$-edge if for its reversed edge $\tau \rightarrow \sigma$ we have

$$
\tau \rightarrow \sigma \in A \text {. }
$$

2. We call $A$ a matching on $X$, if each cell $\sigma \in X^{(*)}$ occurs in at most one edge of $A$.

3. We call $A$ an acyclic matching on $X$, if $A$ is a matching and if the induced graph $G_{X}^{A}$ is acyclic, that is, contains no directed cycle.

4. A cell of $X$ is called $A$-critical if it does not occur in any edge $\tau \rightarrow \sigma \in$ $A$.

5. We set

$$
X_{\text {critical }}^{(*)}(A):=\left\{\sigma \in X^{(*)} \mid \sigma \text { is } A \text {-critical }\right\}
$$

6. We denote by $\tilde{G}_{X}^{A}=\left(X^{(*)}, \tilde{E}_{X}^{A}\right)$ the induced graph with edge set

$$
\tilde{E}_{X}^{A}:=E_{X} \cup\{\sigma \rightarrow \tau \mid \tau \rightarrow \sigma \in A\}
$$

that is built from $G_{X}$ by adding for all edges $\tau \rightarrow \sigma$ that lie in $A$ their reversed edges $\sigma \rightarrow \tau$.

Lemma 3.2.3. Let $X$ be a $C W$-complex, $A$ an acyclic matching on $X$ and $A^{\prime} \subset A$. Then $A^{\prime}$ is an acyclic matching on $X$. 
Proof. Suppose

$$
\gamma=v_{0} \rightarrow \ldots \rightarrow v_{k}, v_{k}=v_{0}
$$

is a cycle in $A^{\prime}$. Note that no two consecutive edges $v_{i} \rightarrow v_{i+1}$ and $v_{i+1} \rightarrow v_{i+2}$ can be $A^{\prime}$-edges, because $A^{\prime}$ is a matching. Since the dimension of the cells involved decreases strictly along edges that are not $A^{\prime}$-edges and increases by 1 along $A^{\prime}$-edges it follows that

$$
\gamma=\tau_{0} \rightarrow \sigma_{0} \rightarrow \ldots \rightarrow \tau_{k} \rightarrow \sigma_{k} \rightarrow \tau_{k+1}, \tau_{k+1}=\tau_{0}
$$

where all

$$
\sigma_{i} \rightarrow \tau_{i+1}, i=0, \ldots, k
$$

are $A^{\prime}$-edges and all

$$
\tau_{i} \rightarrow \sigma_{i}, i=0, \ldots, k
$$

are not $A^{\prime}$-edges (or the other way around). It follows that $\gamma$ also is a cycle in $G_{X}^{A}$, since none of the edges involved that are not $A^{\prime}$-edges can be an $A$-edge (this would violate the property of $A$ of being a matching). Consequently $A^{\prime}$ must be acyclic.

Lemma 3.2.4. Let $X$ be a $C W$-complex, $A$ an acyclic matching on $X$. Then the only cycles in the graph $\tilde{G}_{X}^{A}$ consist of exactly one edge $e \in A$ and its reversed edge.

Proof. If $\gamma$ is a cycle in $\tilde{G}_{X}^{A}$, it must contain one edge $e \in A$ and its reversed edge. (Reason: If not, $\gamma$ can be viewed as a cycle in $G_{X}^{A^{\prime}}$ for some $A^{\prime} \subset A$. This conflicts with Lemma 3.2.3.) These two edges cut $\gamma$ into two parts that again are cycles, so by induction $\gamma$ only consists of pairs of oppositely directed edges. From the fact that $A$ is a matching it follows that $\gamma$ consists of only one such pair.

The above Lemma justifies the following

Definition 3.2.5 (The matching poset $\left(A^{(*)}, \preceq_{A}\right)$ ). Let $X$ be a CW- complex, $A$ an acyclic matching on $X$. 
1. We define

$$
A^{(*)}:=A \cup X_{\text {critical }}^{(*)}(A) .
$$

For $a, b \in A^{(*)}$, we set $a \preceq_{A} b: \Leftrightarrow$ there exists a path in $\tilde{G}_{X}^{A}$ from $b$ to $a$. Here, if $a=\tau \rightarrow \sigma \in A$, we mean a path in $\tilde{G}_{X}^{A}$ from $b$ to either $\sigma$ or $\tau$, if $b=\tau \rightarrow \sigma \in A$, we mean a path in $G_{X}^{A}$ from either $\sigma$ or $\tau$ to $a$. We call the partially ordered set $\left(A^{(*)}, \preceq_{A}\right)$ the matching poset of $A$.

2. We call the function

$$
\begin{aligned}
\operatorname{gr}_{A}: X^{(*)} & \longrightarrow A^{(*)} \\
\sigma & \longmapsto\left\{\begin{array}{rlll}
\sigma & \text { if } & \sigma \in X_{\text {critical }}^{(*)} \\
\tau^{\prime} \rightarrow \tau & \text { if } & \sigma \in\left\{\tau, \tau^{\prime}\right\} \text { and } \tau^{\prime} \rightarrow \tau \in A
\end{array}\right.
\end{aligned}
$$

the $A$-universal grading of $X$. We denote the subcomplexes $X_{\preceq a}$ that arise from this $A^{(*)}$-grading by $X_{\preceq_{A} a}$.

\subsubsection{The correspondence between discrete Morse func- tions and acyclic matchings}

We present the following definitions and lemmas to clarify the connection between discrete Morse functions and acyclic matchings.

Definition 3.2.6. Let $X$ be a CW-complex, $G_{X}=\left(X^{(*)}, E_{X}\right)$ its cell graph and $f: X^{(*)} \longrightarrow \mathbb{R}$ a discrete Morse function on $X$. We set

$$
A_{f}:=\left\{\tau \rightarrow \sigma \in E_{X} \mid \sigma \text { is a facet of } \tau, f(\tau) \leq f(\sigma)\right\} .
$$

We call $A_{f}$ the acyclic matching on $X$ corresponding to $f$.

The above denotation is justified by

Lemma 3.2.7. Let $X$ be a $C W$-complex, $f: X^{(*)} \longrightarrow \mathbb{R}$ a discrete Morse function on $X$. Then

1. $A_{f}$ is an acyclic matching and

2. $X_{\text {critical }}^{(*)}\left(A_{f}\right)=X_{\text {critical }}^{(*)}(f)$. 
This also justifies to denote the set of critical cells just by $X_{\text {critical }}^{(*)}$ as long as only one acyclic matching and its corresponding discrete Morse functions is involved.

Proof. 1. By [13], Lemma 2.5, not both conditions (3.1.3.3) and (3.1.3.4) of Definition 3.1.3 can be violated for a single cell $\sigma \in X^{(*)}$. This proves that all cells $\sigma \in X^{(*)}$ occur in at most one edge of $A_{f}$, so $A_{f}$ is a matching. Acyclicity of $A_{f}$ comes from the fact that $f$ decreases on paths in $G_{X}^{A}$ and decreases even strictly along edges $\sigma \rightarrow \tau$ that are not $A$-edges, of which at least one must occur in any cycle.

2. Obvious from the construction of $A_{f}$.

Lemma 3.2.8. Let $X$ be a $C W$-complex, $A$ an acyclic matching on $X$. Then there exists a discrete Morse function on $X$ such that

$$
A=A_{f}
$$

Proof. Let $\prec$ be any linear extension of $\prec_{A}$ and

$$
f^{(*)}: A^{(*)} \longrightarrow \mathbb{R}
$$

strictly $\prec$-order preserving, that is, for $a, b \in A^{(*)}$ we have

$$
a \prec b \Longrightarrow f^{(*)}(a)<f^{(*)}(b) \text {. }
$$

We define the discrete Morse function $f: X^{(*)} \longrightarrow \mathbb{R}$ by

$$
f(\sigma)=\left\{\begin{array}{lll}
f^{*}(a) & \text { if } & \sigma=a \in X_{\text {critical }}^{(*)} \\
f^{*}(a) & \text { if } & a=\tau \rightarrow v \in A \text { and } \sigma \in\{\tau, v .\}
\end{array}\right.
$$

It is easy to check that $f$ is a discrete Morse function on $X$ and $A_{f}=A$.

The above leads to the following reformulation of Theorem 3.1.4:

Theorem 3.2.9. Let $X$ be a finite $C W$-complex, $A$ an acyclic matching on $X$. Then there is a $C W$-complex $X_{A}$ whose $i$-cells are in one-to-one correspondence with the $A$-critical $i$-cells of $X$ such that $X_{A}$ is homotopy equivalent to $X$. 


\subsubsection{Discrete Morse theory for graded CW-complexes}

In the remainder of this section we give an explicit description of the Morse complex $X_{A}$ corresponding to an acyclic matching $A$ on $X$, extend the theory to not necessarily finite CW-complexes and show that the Morse equivalence is, in a canonical way, compatible with gradings.

Definition 3.2.10. Let $P$ be a partially ordered set, $(X, \mathrm{gr})$ a $P$-graded CW-complex.

1. We call the grading gr : $X^{(*)} \longrightarrow P$ a compact $\mathrm{P}$-grading of $X$ if $X_{\preceq p}$ is compact for all $p \in P$. $X$ is then called compactly $P$-graded.

2. We call an acyclic matching $A$ of $X$ proper if the corresponding $A$ universal grading $\operatorname{gr}_{A}: X^{(*)} \longrightarrow A^{(*)}$ is compact.

We now make some preparations for the construction of the Morse complex $X_{A}$ corresponding to a proper acyclic matching $A$ on $X$ :

Lemma 3.2.11. Let $X$ be a $C W$-complex.

1. Let $\sigma, \tau \in X^{(*)}, \operatorname{dim}=i, \operatorname{dim} \tau=i+1, \sigma$ a regular face of $\tau$. Then there exists a deformation retraction

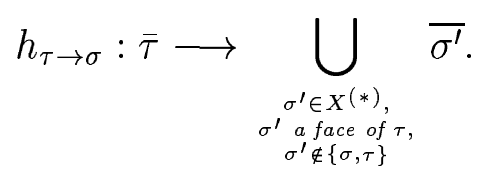

2. Let $A$ be an acyclic matching on $G_{X}, a \in A^{(*)}$. If $a=(\tau \rightarrow \sigma) \in A$, then

$$
\begin{aligned}
\tilde{h}_{\tau \rightarrow \sigma}: X_{\preceq_{A} a} & \longrightarrow X_{\prec_{A} a} \\
x & \longmapsto\left\{\begin{array}{cll}
x & \text { if } & x \notin \bar{\tau} \\
h_{\tau \rightarrow \sigma}(x) & \text { if } & x \in \bar{\tau},
\end{array}\right.
\end{aligned}
$$

is a deformation retraction.

Proof. Obvious from Definition 3.1.1 
Definition 3.2.12. Let $A, X, Y$ and $Y^{\prime}$ be topological spaces, $A \subset X, \phi$ : $Y \longrightarrow Y^{\prime}$ and $f: A \longrightarrow Y$ continuous maps. We denote by

$$
\operatorname{id} \cup_{f} \phi: X \cup_{f} Y \longrightarrow X \cup_{\phi \circ f} Y^{\prime}
$$

the unique map that makes the following diagram commutative:

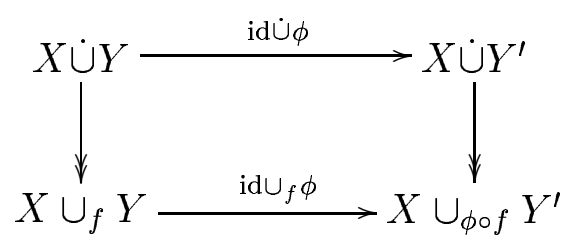

Definition 3.2.13 (Morse complex, Morse equivalence). Let $X$ be a CW-complex, $A$ a proper acyclic matching on $X$.

Recall (see Definition 3.2.5) that $\preceq_{A}$ denotes the partial order of the matching poset $A^{(*)}$. For all $a \in A^{(*)}$ we define inductively a CW-complex $\left(X_{A}\right)_{\preceq_{A} a}, a \in A^{(*)}$ and a map

$$
H(A)_{\preceq_{A} a}: X_{\preceq_{A} a} \longrightarrow\left(X_{A}\right)_{\preceq_{A} a}:
$$

1. Let $a \in A^{(*)}$ be $\prec_{A}$-minimal. We set

$$
\left(X_{A}\right)_{\preceq_{A} a}:=X_{\preceq_{A} a}=a
$$

(note that $a \prec_{A}$-minimal $\Rightarrow a \in X_{\text {critical }}^{(*)}$ ) and

$$
H(A)_{\preceq_{A} a}:=i d_{X_{\unlhd_{A} a}} .
$$

2. Let $a \in A^{(*)}$. Suppose for all $b \prec_{A} a$, the CW-complexes $\left(X_{A}\right)_{\preceq_{A} b}$ and the maps $H(A)_{\preceq_{A} b}: X_{\preceq_{A} b} \longrightarrow\left(X_{A}\right)_{\preceq_{A} b}$ are constructed such that for all $b, b^{\prime} \in A^{(*)}, b^{\prime} \preceq_{A} b \prec_{A} a$, we have

$$
\left(X_{A}\right)_{\preceq_{A} b^{\prime}} \subset\left(X_{A}\right)_{\preceq_{A} b}
$$

and the diagram

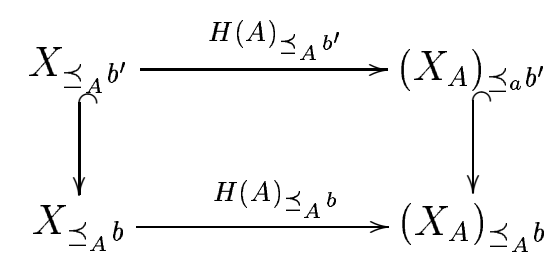


commutes.

Denote by $\left(X_{A}\right)_{\prec_{A} a}$ the union

$$
\left(X_{A}\right)_{\prec_{A} a}:=\bigcup_{b \prec_{A} a}\left(X_{A}\right)_{\preceq_{A} b}
$$

and by

$$
H(A)_{\prec_{A} a}: X_{\prec_{A} a} \longrightarrow\left(X_{A}\right)_{\prec_{A} a}
$$

the map induced by the maps

$$
H(A)_{\preceq_{A} b}: X_{\preceq_{A} b} \longrightarrow\left(X_{A}\right)_{\preceq_{A} b}, b \prec_{A} a .
$$

- Case 1: $a=\tau \rightarrow \sigma \in A$

We set

$$
\left(X_{A}\right)_{\preceq_{A} a}:=\left(X_{A}\right)_{\prec_{A} a}
$$

and define

$$
H(A)_{\preceq_{A} a}: X_{\preceq_{A} a} \longrightarrow\left(X_{A}\right)_{\preceq_{A} a}
$$

by

$$
H(A)_{\preceq_{A} a}:=H(A)_{\prec_{A} a} \circ \tilde{h}_{\tau \rightarrow \sigma} .
$$

- Case 2: $a=\sigma \in X_{\text {critical }}^{(*)}$

Let $\operatorname{dim} \sigma=i$ and $f_{\sigma}: B_{\sigma}^{i} \longrightarrow X_{\preceq_{A} a}$ be the characteristic map of the cell $\sigma$. Recall that

$$
X_{\preceq_{A} a}=B_{\sigma}^{i} \cup_{f_{\partial \sigma}} X_{\prec_{A} a} .
$$

We set

$$
\left(X_{A}\right)_{\swarrow_{A} a}:=B_{\sigma}^{i} \cup_{H(A) \prec_{A} a^{\circ} f_{\partial \sigma}}\left(X_{A}\right)_{\prec_{A} a}
$$

and

$$
H(A)_{\preceq_{A} a}:=\operatorname{id}_{B_{\sigma}^{i}} \cup_{f_{\partial \sigma}} H(A)_{\prec_{A} a} .
$$


We define

$$
X_{A}:=\bigcup_{a \in A^{(*)}}\left(X_{A}\right) \preceq_{A} a
$$

and

$$
H(A): X \longrightarrow X_{A}
$$

to be the map induced by the maps

$$
H(A)_{\preceq_{A} a}: X_{\preceq_{A} a} \longrightarrow\left(X_{A}\right)_{\preceq_{A} a} .
$$

We call $X_{A}$ the Morse complex of $X$ with respect to $A$ and $H(A)$ the corresponding Morse equivalence.

Theorem 3.2.14. Let $X$ be a $C W$-complex, $A$ a proper acyclic matching on $X$. Let $X_{A}$ be the corresponding Morse complex, $H(A): X \longrightarrow X_{A}$ the corresponding Morse equivalence. Then

1. for all $i \in \mathbb{N}$, the $i$-cells of $X_{A}$ are in one-to-one correspondence with the $A$-critical $i$-cells of $X$ and

2. the Morse equivalence $H(A): X \longrightarrow X_{A}$ is a homotopy equivalence.

Furthermore, there is a canonical $A^{(*)}$-grading $\operatorname{gr}_{A}: X_{A}^{(*)} \longrightarrow A^{(*)}$ of $X_{A}$ given by the composition

$$
X_{A}^{(*)} \stackrel{\cong}{\longrightarrow} X_{\text {critical }}^{(*)} \hookrightarrow X^{(*)} \stackrel{\mathrm{gr}_{A}}{\longrightarrow} A^{(*)} .
$$

For all $a \in A^{(*)}$ the subcomplex $\left(X_{A}\right)_{\preceq a}$ with respect to this $A^{(*)}$-grading is given by the subcomplex

$$
\left(X_{A}\right)_{\preceq a}=\left(X_{A}\right)_{\preceq_{A} a}
$$

from Definition 3.2.13. Also the Morse equivalence $H(A): X \longrightarrow X_{A}$ respects these $A^{(*)}$-gradings, that is:

3. For all $a \in A^{(*)}$ and all $i \in \mathbb{N}$, the $i$-cells of $\left(X_{A}\right)_{\varsigma_{A} a}$ are in one-to-one correspondence with the $A$-critical $i$-cells of $X_{\unlhd_{A} a}$ and 
4. for all $a \in A^{(*)}$ the restriction $H(A)_{\preceq_{A} a}=\left.H(A)\right|_{X_{\unlhd_{A} a}}$ is a homotopy equivalence

$$
H(A)_{\preceq_{A} a}: X_{\preceq_{A} a} \stackrel{\simeq}{\longrightarrow}\left(X_{A}\right)_{\preceq_{A} a}:
$$

Proof. The fact that the $A$-universal grading gr ${ }_{A}: X^{(*)} \longrightarrow A^{(*)}$ of $X$ induces a canonical $A^{(*)}$-grading $\operatorname{gr}_{A}: X_{A}^{(*)} \longrightarrow A^{(*)}$ of $X_{A}$ can be seen from the definition of the Morse complex (see Definition 3.2.13) which is by done induction over the matching poset $A^{(*)}$ : Every new cell $\sigma_{A} \in X_{A}^{(*)}$ corresponding to a critical cell $\sigma \in X_{\text {critical }}^{(*)}$, say $\operatorname{gr}_{A}(\sigma)=a$ is attached to the complex $\left(X_{A}\right)_{\prec_{A} a}$ so that for all faces $\tau_{A} \in X_{A}$ of $\sigma_{A}$ corresponding to critical cells $\tau \in X_{\text {critical }}^{(*)}$ we have that $\operatorname{gr}_{A}(\tau) \prec_{A} \operatorname{gr}_{A}(\sigma)$.

Also parts 1. and 3. are immediate consequences of the inductive construction of the Morse complex: For every element $a \in A^{(*)}$ that is a critical cell $a=\sigma \in X_{\text {critical }}^{(*)}$, we attach a cell of the same dimension. For every element $a \in A^{(*)}$ that is an element $a=\tau \rightarrow \sigma \in A$, we do not attach any cells.

For parts 2. and 4. we use [24]: From Proposition 3.1 (Projection Lemma) and Proposition 3.7 (Homotopy Lemma) we derive the fact, that the induced maps

$$
H(A)_{\preceq_{A} a}: X_{\preceq_{A} a} \longrightarrow\left(X_{A}\right)_{\preceq_{A} a}
$$

and

$$
H(A): X \longrightarrow X_{A}
$$

are homotopy equivalences.

Definition 3.2.15. Let $X$ be a CW-complex, $P$ a partially ordered set, gr : $X^{(*)} \longrightarrow P$ a $P$-grading of $X$ and $A$ an acyclic matching on $X$. We call $A$ homogeneous with respect to the $P$-grading gr $: X^{(*)} \longrightarrow P$ if we have:

$$
\operatorname{gr}(\tau)=\operatorname{gr}(\sigma) \text { for all } \tau \rightarrow \sigma \in A .
$$

Remark 3.2.16. Let $X$ be a CW-complex, $P$ a partially ordered set, gr : $X^{(*)} \longrightarrow P$ a $P$-grading of $X$ and $A$ an acyclic matching on $X$. Then the following are equivalent: 
1. $A$ is homogeneous with respect to the $P$-grading $g r: X^{(*)} \longrightarrow P$,

2. the $A$-universal grading of $X$ is compatible with the $P$-grading gr : $X^{(*)} \longrightarrow P$, that is, there exists an order preserving map $g: A^{(*)} \longrightarrow P$ such that the diagram

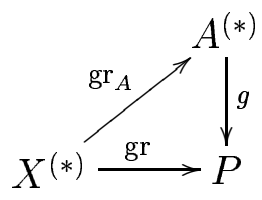

commutes.

This is why we call $\operatorname{gr}_{A}: X^{(*)} \longrightarrow A^{(*)}$ the universal $A$-grading of $X$.

Remark 3.2.17. Let $X$ be a CW-complex, $P$ a partially ordered set, gr : $X^{(*)} \longrightarrow P$ a compact $P$-grading of $X$ and $A$ an acyclic matching on $X$ which is homogeneous with respect to gr: $X^{(*)} \longrightarrow P$. Then $A$ is proper.

Proof. We use again the following commutative diagram:

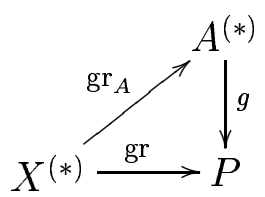

If gr : $X^{(*)} \longrightarrow P$ is compact then so is the $A$-universal grading $\operatorname{gr}_{A}: X^{(*)} \longrightarrow$ $A^{(*)}$.

Corollary 3.2.18. Let $X$ be a $C W$-complex, $P$ a partially ordered set, gr : $X^{(*)} \longrightarrow P$ a compact $P$-grading of $X$ and $A$ an acyclic matching on $X$ which is homogeneous with respect to gr: $X^{(*)} \longrightarrow P$. Then the $P$-grading gr $: X^{(*)} \longrightarrow P$ of $X$ induces a compact $P$-grading gr $: X_{A}^{(*)} \longrightarrow P$ of the Morse complex $X_{A}$ and the Morse equivalence $H(A): X \longrightarrow X_{A}$ respects these P-gradings, that is:

1. For all $p \in P$ and all $i \in \mathbb{N}$, the $i$-cells of $\left(X_{A}\right)_{\preceq p}$ are in one-to-one correspondence with the $A$-critical $i$-cells of $X_{\preceq p}$ and 
2. for all $p \in P$ the restriction $H(A)_{\preceq p}:=\left.H(A)\right|_{X_{\preceq p}}$ is a homotopy equivalence

$$
H(A)_{\preceq p}: X_{\preceq p} \stackrel{\simeq}{\longrightarrow}\left(X_{A}\right)_{\preceq p}
$$

Proof. There is the following commutative diagram:

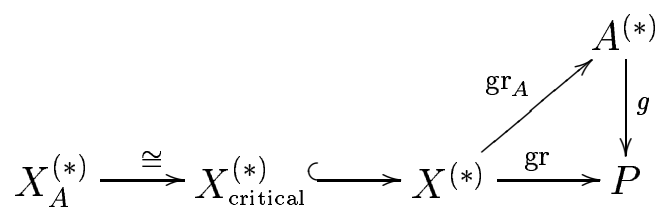

Here, the composition gr of the maps in the lower row is the induced $P$ grading of $X_{A}$ which is order preserving because it factorizes into

$$
\operatorname{gr}=g \circ \operatorname{gr}_{A}
$$

where $\operatorname{gr}_{A}: X_{A}^{(*)} \longrightarrow A^{(*)}$ is the canonical $A^{(*)}$-grading of $X_{A}$.

To prove the remaining assertions, note that

$$
X_{\preceq p}=\bigcup_{\substack{a \in A^{(*)}, g(a) \preceq p}} X_{\preceq_{A} a}
$$

and

$$
\left(X_{A}\right)_{\preceq p}=\bigcup_{\substack{a \in A^{(*)} \\ g(a) \preceq p}}\left(X_{A}\right)_{\preceq_{A} a} .
$$

Now we use again [24]: From Proposition 3.1 (Projection Lemma) and Proposition 3.7 (Homotopy Lemma) it follows that the induced maps

$$
H(A)_{\preceq p}: X_{\preceq p} \longrightarrow\left(X_{A}\right)_{\preceq p}
$$

are homotopy equivalences. 


\subsection{Discrete Morse theory for cellular reso- lutions}

Now we are in position to formulate the main result of this chapter which makes discrete Morse theory applicable to cellular resolutions.

Theorem 3.3.1. Let $R=k[\Lambda]$ be an affine semigroup ring and $M a \mathbb{Z}^{d_{-}}$ graded $R$-module. Assume $(X, \mathrm{gr})$ is a compactly $\left(\mathbb{Z}^{d}, \Lambda\right)$-graded $C W$-complex which supports a cellular resolution $\mathcal{F}_{X}^{\mathrm{gr}}$ of $M$. Then for a homogeneous acyclic matching $A$ of $X$ the $\left(\mathbb{Z}^{d}, \Lambda\right)$-graded $C W$-complex $\left(X_{A}\right.$, gr) supports a cellular resolution $\mathcal{F}_{X_{A}}^{\mathrm{gr}}$ of $M$.

For the proof of Theorem 3.3.1 we need the following lemmas:

Lemma 3.3.2. Let $\mathcal{C}_{X}^{\mathrm{gr}}$ be a cellular complex supported by the $\left(\mathbb{Z}^{d}, \Lambda\right)$-graded $C W$-complex $X$. Then, for $i \geq 0$ and $\alpha \in \mathbb{Z}^{d}$

$$
H_{i}\left(\mathcal{C}_{X}^{\mathrm{gr}}\right)_{\alpha} \cong H_{i}\left(X_{\preceq \alpha} ; k\right),
$$

as k-vectorspaces.

Proof. For a fixed $\alpha$ an $i$-cell $c \in X^{(*)}$ gives rise to an $\alpha$-homogeneous piece $\underline{\mathbf{x}}^{\alpha-\gamma} e_{c}, \gamma=\operatorname{gr}(c)$, in homological degree $i$ if and only if $\operatorname{gr}(c)=\gamma \preceq \alpha$. Therefore, as a sequence of $k$-vectorspaces, $\mathcal{C}_{\alpha}$ is isomorphic to the cell-homology chain complex of $X_{\preceq \alpha}$, which proves the assertion.

Proposition 3.3.3. Let $\mathcal{C}_{X}^{\mathrm{gr}}$ be the cellular complex supported by the compactly $\left(\mathbb{Z}^{d}, \Lambda\right)$-graded $C W$-complex $X$. Let $A$ be a homogeneous acyclic matching on $X$. Then $\left(X_{A}\right.$, gr) supports a cellular $\mathbb{Z}^{d}$-graded chain complex $\mathcal{C}_{X_{A}}^{\text {gr }}$ such that $H_{i}\left(\mathcal{C}_{X_{A}}^{\mathrm{gr}}\right) \cong H_{i}\left(\mathcal{C}_{X}^{\mathrm{gr}}\right), i \geq 0$.

Proof. By Lemma 3.3.2 we have

$$
H_{i}\left(\mathcal{C}_{X_{A}}^{\mathrm{gr}}\right)_{\alpha} \cong H_{i}\left(\left(X_{A}\right)_{\preceq \alpha} ; k\right), H_{i}\left(\mathcal{C}_{X}^{\mathrm{gr}}\right)_{\alpha} \cong H_{i}\left(X_{\preceq \alpha} ; k\right) .
$$

By Corollary 3.2.18 $H_{i}\left(X_{\preceq \alpha} ; k\right) \cong H_{i}\left(\left(X_{A}\right)_{\preceq \alpha} ; k\right)$ and the assertion follows.

The proof of Theorem 3.3.1 now follows easily. 
Proof. Since $\mathcal{F}_{X}^{\mathrm{gr}}$ is a resolution of $M$ we have $H_{i}\left(\mathcal{C}_{X}^{\mathrm{gr}}\right)=0$ for $i \geq 1$ and $H_{0}\left(\mathcal{C}_{X}^{\mathrm{gr}}\right)=M$. Now by Proposition 3.3.3 $X_{A}$ defines a cellular chain complex with $H_{i}\left(\mathcal{C}_{X_{A}}^{\text {gr }}\right)=H_{i}\left(\mathcal{C}_{X}^{\text {gr }}\right)=0, i \geq 1$ and $H_{0}\left(\mathcal{C}_{X_{A}}^{\text {gr }}\right)=H_{0}\left(\mathcal{C}_{X}^{\text {gr }}\right)=M$. Thus $X_{A}$ and gr support a $\mathbb{Z}^{d}$-graded free resolution of $M$ as an $R$-module.

\subsection{Morse differentials}

For a finite CW-complex, Forman presents in [13], Chapter 8, a differential complex

$$
\mathcal{M}=\stackrel{\tilde{\partial}_{p+1}}{\longrightarrow} \ldots \mathcal{M}_{p} \stackrel{\tilde{\partial}_{p}}{\longrightarrow} \mathcal{M}_{p-1} \longrightarrow \ldots \longrightarrow \mathcal{M}_{0}
$$

with the following properties:

1. For all $p \in \mathbb{N}$ the module $\mathcal{M}_{p}$ is the free module generated by the critical p-cells of $X$.

2. The homology of $\mathcal{M}$ is the same as the homology of the underlying CW-complex $X$.

In Theorem 8.10, he gives an explicit formula for the differential of this complex. In this section we confirm that, for the corresponding acyclic matching $A$, the differential complex presented by Forman in fact coincides with the cellular chain complex corresponding to the Morse complex $X_{A}$. This enables us to present in Chapter 4 explicit formulas for the differential of resolutions derived via discrete Morse theory for cellular resolutions.

Definition 3.4.1. Let $X$ be a CW-complex, $A$ a proper acyclic matching on $X$ and $X_{A}$ the corresponding Morse complex.

1. We denote by

$$
\mathcal{C}^{A}=\ldots \stackrel{\partial_{i+1}^{A}}{\longrightarrow} \mathcal{C}_{i}\left(X_{A}\right) \stackrel{\partial_{i}^{A}}{\longrightarrow} \mathcal{C}_{i-1}\left(X_{A}\right) \stackrel{\partial_{i-1}^{A}}{\longrightarrow} \ldots \stackrel{\partial_{1}^{A}}{\longrightarrow} \mathcal{C}_{0}\left(X_{A}\right)
$$

the cellular chain complex of $X_{A}$ and by [ : $]_{A}$ the corresponding coefficients of the differential $\partial^{A}$, that is, for $\tau \in X_{A}^{(*)}$ we have

$$
\partial^{A}(\tau)=\sum_{\sigma \text { facet of } \tau}[\sigma: \tau]_{A} \sigma .
$$


2. For $\sigma \in X^{(*)}, \sigma^{\prime} \in X_{\text {critical }}^{(*)}$, we define

$$
\Gamma_{A}\left(\sigma, \sigma^{\prime}\right):=\left\{\gamma \mid \gamma \text { is a path in } G_{X}^{A} \text { from } \sigma \text { to } \sigma^{\prime}\right\} .
$$

3. For $\sigma \in X^{(*)}, \sigma^{\prime} \in X_{\text {critical }}^{(*)}, \operatorname{dim} \sigma=\operatorname{dim} \sigma^{\prime}=i$,

$$
\gamma=\sigma_{0} \rightarrow \tau_{1} \rightarrow \sigma_{1} \rightarrow \ldots \rightarrow \sigma_{k-1} \rightarrow \tau_{k} \rightarrow \sigma_{k} \in \Gamma_{A}\left(\sigma, \sigma^{\prime}\right)
$$

(that is, $\sigma_{0}=\sigma, \sigma_{k}=\sigma^{\prime}, \operatorname{dim} \sigma_{j}=i, \operatorname{dim} \tau_{j}=i+1$ and $\tau_{j} \rightarrow \sigma_{j-1} \in A$ for all $j=1, \ldots, k$ ), we set

$$
m(\gamma):=(-1)^{k} \prod_{i=1}^{k}\left[\sigma_{i-1}: \tau_{i}\right] \cdot\left[\sigma_{i}: \tau_{i}\right]
$$

Theorem 3.4.2. Let $X$ be a $C W$-complex, $A$ a proper acyclic matching on $X$ and $X_{A}$ the corresponding Morse complex. Let $\sigma, \tau \in X_{\text {critical }}^{(*)}, \sigma_{A}, \tau_{A} \in X_{A}^{(*)}$ their corresponding cells in $X_{A}^{(*)}$. Then

$$
\left[\sigma_{A}: \tau_{A}\right]_{A}=\sum_{\sigma_{\text {facet of } \tau}}\left[\sigma^{\prime}: \tau\right] \sum_{\gamma \in \Gamma_{A}\left(\sigma^{\prime}, \sigma\right)} m(\gamma)
$$

For the proof of Theorem 3.4.2 we need the following lemmas:

Lemma 3.4.3. Let $X$ be a $C W$-complex, $\sigma_{0}, \tau_{0} \in X^{(*)}, A=\left\{\tau_{0} \rightarrow \sigma_{0}\right\} a$ proper (acyclic) matching on $X$ and $X_{A}$ the corresponding Morse complex. Let $\sigma, \tau \in X_{\text {critical }}^{(*)}, \sigma_{A}, \tau_{A} \in X_{A}^{(*)}$ their corresponding cells in $X_{A}$. If $\sigma$ is not a facet of $\tau_{0}$ or $\sigma_{0}$ is not a facet of $\tau$, then

$$
\left[\sigma_{A}: \tau_{A}\right]_{A}=[\sigma: \tau]
$$

If $\sigma$ is a facet of $\tau_{0}$ and $\sigma_{0}$ is a facet of $\tau$, then

$$
\left[\sigma_{A}: \tau_{A}\right]_{A}=[\sigma: \tau]-\left[\sigma_{0}: \tau\right] \cdot\left[\sigma: \tau_{0}\right]
$$

Proof. Note that, from Definition 3.2.13, for all critical cells $\sigma \in X_{\text {critical }}^{(*)}$, the restriction of the Morse equivalence $H(A)$ to $\sigma$ is a homeomorphism onto the cell $\sigma_{A}$ in the Morse complex $X_{A}$ :

$$
\left.H(A)\right|_{\sigma}: \sigma \stackrel{\cong}{\longrightarrow} \sigma_{A}
$$


These homeomorphisms induce an inclusion

$$
i_{A}^{j}: X_{A}^{j} \hookrightarrow X^{j}
$$

of the $j$-skeletons. For every $j$-cell $\sigma \in X^{(j)}$, we get the following commutative diagram, where $p_{\sigma}: X_{A}^{j} \longrightarrow S^{j}$ is the cellular projection map for the cell $\sigma$ (see Definition 2.1.13):

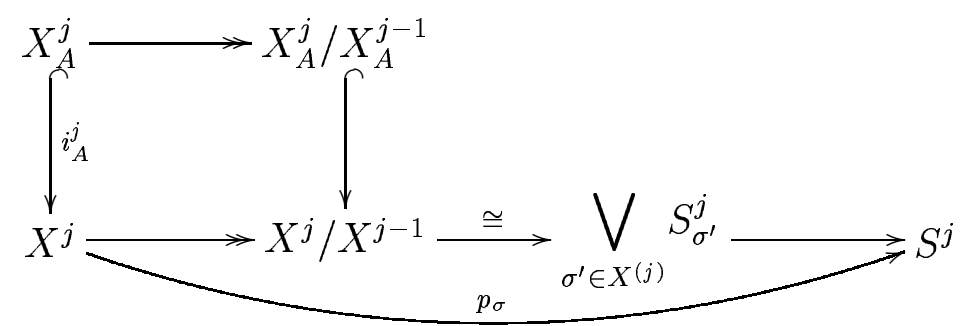

Note that, although $i_{A}^{j}$ is not continuous in general, the map

$$
X_{A}^{j} / X_{A}^{j-1} \hookrightarrow X^{j} / X^{j-1}
$$

is. For $\sigma_{A} \in X_{A}^{(j)}$, we define $p_{\sigma_{A}}: X_{A}^{j} \longrightarrow S^{j}$ by

$$
p_{\sigma_{A}}:=p_{\sigma} \circ i_{A}^{j}
$$

For $\sigma, \sigma^{\prime} \in X_{\text {critical }}^{(*)}$, we get the following commutative diagram:

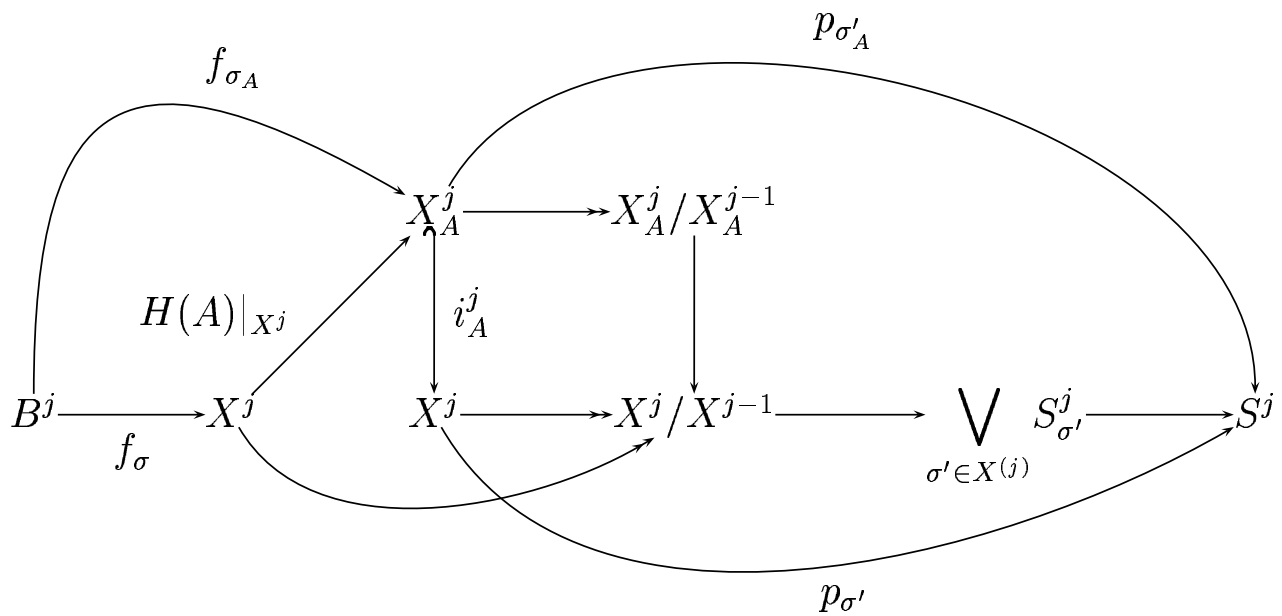


Here, $f_{\sigma}$ and $f_{\sigma_{A}}$ are the characteristic maps for the cells $\sigma$ and $\sigma_{A}$. Note that, although this diagram cannot be extended commutatively by adding the identity map id $: X^{j} \longrightarrow X^{j}$, it is commutative with both maps $X^{j} \longrightarrow$ $X^{j} / X^{j-1}$ defined as the canonical projection maps. We therefore get

$$
p_{\sigma_{A}^{\prime}} \circ f_{\sigma_{A}}=p_{\sigma^{\prime}} \circ f_{\sigma} .
$$

This proves that the $p_{\sigma_{A}}$ define cellular projection maps for the cells $\sigma_{A} \in$ $X_{A}^{(*)}$.

Now we are ready to calculate, for critical cells $\sigma, \tau \in X_{\text {critical }}^{(*)}, \operatorname{dim} \sigma=$ $j, \operatorname{dim} \tau=j+1$, the coefficients $\left[\sigma_{A}: \tau_{A}\right]_{A}$. For this, we consider the following commutative diagram:

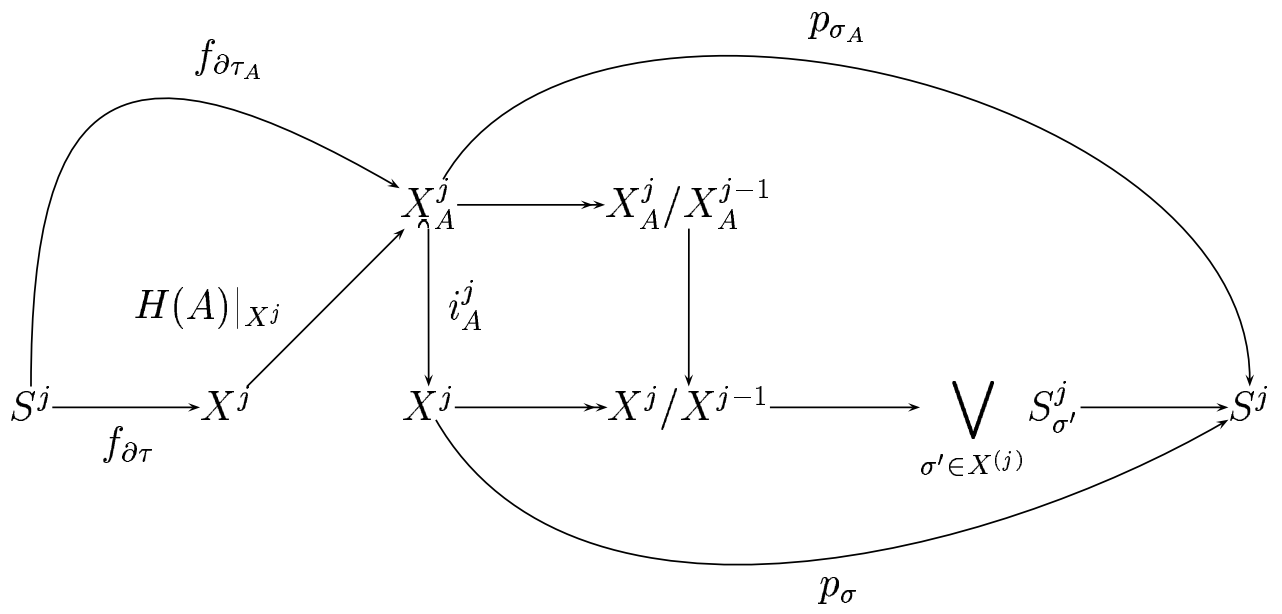

Using this diagram, straightforward considerations show that if $\sigma$ is not a facet of $\tau_{0}$ or $\sigma_{0}$ is not a facet of $\tau$, then we have

$$
p_{\sigma_{A}} \circ f_{\partial \tau_{A}}=p_{\sigma} \circ f_{\partial \tau}
$$

and therefore

$$
\left[\sigma_{A}: \tau_{A}\right]_{A}=[\sigma: \tau]
$$

Let now $\sigma, \tau \in X_{\text {critical }}^{(*)}, \operatorname{dim} \sigma=j, \operatorname{dim} \tau=j+1$, such that $\sigma$ is a facet of $\tau_{0}$ and $\sigma_{0}$ is a facet of $\tau$. We get the following commutative diagram: 




Here, $\Psi$ is defined to be the unique map such that the diagram

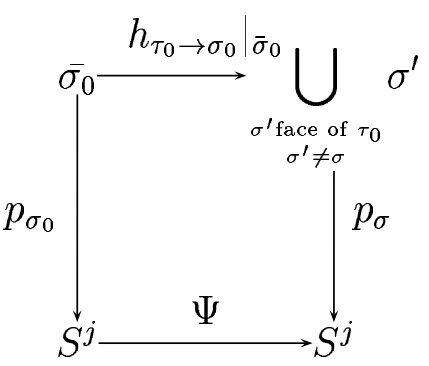

commutes. The fact that $\sigma_{0}$ is a regular face of $\tau_{0}$ shows that

$$
\operatorname{deg} \Psi=-\left[\sigma_{0}: \tau_{0}\right] \cdot\left[\sigma: \tau_{0}\right] .
$$

It follows

$$
\left[\sigma_{A}: \tau_{A}\right]_{A}=[\sigma: \tau]-\left[\sigma_{0}: \tau\right] \cdot\left[\sigma: \tau_{0}\right]
$$


Lemma 3.4.4. Let $X$ be a $C W$-complex, $\sigma, \tau \in X^{(*)}, A$ an acyclic matching on $X, \tau \rightarrow \sigma \in A$ such that $\tau \rightarrow \sigma$ is $\prec_{A}$-minimal in $A$. Let $A^{\prime}:=A-$ $\{\tau \rightarrow \sigma\}, X_{A}$ and $X_{A^{\prime}}$ the corresponding Morse complexes. Then, for the corresponding cells $\sigma_{A^{\prime}}, \tau_{A^{\prime}} \in X_{A^{\prime}}^{(*)}, \sigma_{A^{\prime}}$ is a regular facet of $\tau_{A^{\prime}}$, thus $\left\{\tau_{A^{\prime}} \rightarrow\right.$ $\left.\sigma_{A^{\prime}}\right\}$ is an acyclic matching on $X_{A^{\prime}}$, and for the corresponding Morse complex $\left(X_{A^{\prime}}\right)_{\tau_{A^{\prime}} \rightarrow \sigma_{A^{\prime}}}$ we have

$$
\left(X_{A^{\prime}}\right)_{\tau_{A^{\prime}} \rightarrow \sigma_{A^{\prime}}}=X_{A}
$$

Furthermore, for the Morse equivalences

$$
\begin{aligned}
& H(A): X \stackrel{\simeq}{\longrightarrow} X_{A}, \\
& H\left(A^{\prime}\right): X \stackrel{\simeq}{\longrightarrow} X_{A^{\prime}}
\end{aligned}
$$

and

$$
H\left(\tau_{A^{\prime}} \rightarrow \sigma_{A^{\prime}}\right): X_{A^{\prime}} \stackrel{\simeq}{\longrightarrow}\left(X_{A^{\prime}}\right)_{\tau_{A^{\prime}} \rightarrow \sigma_{A^{\prime}}},
$$

we get the following commutative diagram:

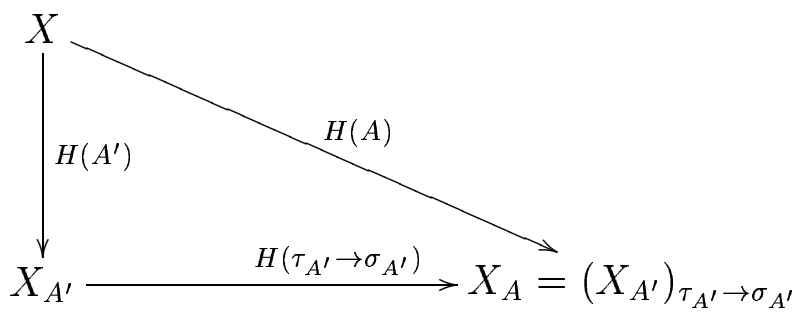

Proof. We use induction over the matching poset $A^{(*)}$. For all $a \preceq_{A} \tau \rightarrow \sigma$, the assertion is trivial. For $\tau \rightarrow \sigma \preceq a$, the assertion follows from the definition of the Morse complex (see Definition 3.2.13).

We are now ready to prove Theorem 3.4.2.

Proof. We can assume that $X_{\text {critical }}^{(*)}$ is finite, since for the coefficient $[\sigma: \tau]$ we only need to consider $X_{\preceq_{A} \tau}$ which are compact. We proceed by induction: Let $\sigma_{0}, \tau_{0} \in X^{(*)}, \tau_{0} \rightarrow \sigma_{0} \in A$ such that $\tau_{0} \rightarrow \sigma_{0}$ is $\prec_{A}$-minimal in $A$. 
Let $A^{\prime}:=A-\left\{\tau_{0} \rightarrow \sigma_{0}\right\}, X_{A}$ and $X_{A^{\prime}}$ the corresponding Morse complexes. Then, by induction hypothesis, we have

$$
\left[\sigma_{A^{\prime}}: \tau_{A^{\prime}}\right]_{A^{\prime}}=\sum_{\sigma^{\prime} \text { facet of } \tau}\left[\sigma^{\prime}: \tau\right] \sum_{\gamma \in \Gamma_{A^{\prime}}\left(\sigma^{\prime}, \sigma\right)} m(\gamma),
$$

where $\Gamma_{A^{\prime}}\left(\sigma^{\prime}, \sigma\right)$ is the set of paths in $G_{X}^{A^{\prime}}$ from $\sigma^{\prime}$ to $\sigma$.

Note that the following are equivalent:

- $\Gamma_{A^{\prime}}\left(\sigma^{\prime}, \sigma_{0}\right)=\emptyset$ for all facets $\sigma^{\prime}$ of $\tau$

- $\sigma_{0 A^{\prime}}$ is not a facet of $\tau_{A^{\prime}}$ or $\sigma_{A^{\prime}}$ is not a facet of $\tau_{0 A^{\prime}}$.

We consider the following cases:

1. If $\sigma_{0 A^{\prime}}$ is not a facet of $\tau_{A^{\prime}}$ or $\sigma_{A^{\prime}}$ is not a facet of $\tau_{0 A^{\prime}}$, we have

$$
\Gamma_{A}\left(\sigma^{\prime}, \sigma\right)=\Gamma_{A^{\prime}}\left(\sigma^{\prime}, \sigma\right)
$$

and, on the other hand, by Lemma 3.4.3, we have

$$
\left[\left(\sigma_{A^{\prime}}\right)_{\tau_{0_{A^{\prime}}} \rightarrow \sigma_{0 A^{\prime}}}:\left(\tau_{A^{\prime}}\right)_{\tau_{0_{A^{\prime}} \rightarrow \sigma_{0} A^{\prime}}}\right]_{\tau_{A_{A^{\prime}} \rightarrow \sigma_{0} A^{\prime}}}=\left[\sigma_{A^{\prime}}: \tau_{A^{\prime}}\right]_{A^{\prime}},
$$

so applying Lemma 3.4.4 shows that the asserted formula for $\left[\sigma_{A}: \tau_{A}\right]_{A}$ is fullfilled.

2. If $\sigma_{0 A^{\prime}}$ is a facet of $\tau_{A^{\prime}}$ and $\sigma_{A^{\prime}}$ is a facet of $\tau_{0 A^{\prime}}$, we have

$$
\Gamma_{A}\left(\sigma^{\prime}, \sigma\right)=\Gamma_{A^{\prime}}\left(\sigma^{\prime}, \sigma\right) \cup\left\{\gamma *\left(\sigma_{0} \rightarrow \tau_{0} \rightarrow \sigma\right) \mid \gamma \in \Gamma\left(\sigma^{\prime}, \sigma_{0}\right)\right\} .
$$

We learn from Lemma 3.4.3 that

$$
\begin{aligned}
{\left[\left(\sigma_{A^{\prime}}\right)_{\tau_{0 A^{\prime}} \rightarrow \sigma_{0} A^{\prime}}\right.} & \left.:\left(\tau_{A^{\prime}}\right)_{\tau_{0 A^{\prime}} \rightarrow \sigma_{0 A^{\prime}}}\right]_{\tau_{0_{A^{\prime}} \rightarrow \sigma_{A^{\prime}}}}= \\
& =\left[\sigma_{A^{\prime}}: \tau_{A^{\prime}}\right]_{A^{\prime}}-\left[\sigma_{0 A^{\prime}}: \tau_{A^{\prime}}\right]_{A^{\prime}} \cdot\left[\sigma_{A^{\prime}}: \tau_{0 A^{\prime}}\right]_{A^{\prime}}
\end{aligned}
$$

Application of Lemma 3.4.4 and considering the definition of $m(\gamma)$ proves the formula. 
We are now able to give the following criterion for minimality:

Corollary 3.4.5. Let $(X$, gr $)$ be a compactly $(\mathbb{Z}, \Lambda)$-graded $C W$-complex which supports a cellular resolution $\mathcal{F}_{X}^{\text {gr }}$. Let $A$ be a homogeneous acyclic matching on $X$. Then $\mathcal{F}_{X_{A}}^{\mathrm{gr}}$ is minimal if there is no triple of cells $\sigma^{\prime} \leq \sigma$ and $\sigma^{\prime \prime}$ of $X$ such that $\sigma, \sigma^{\prime \prime}$ are A-critical, $\operatorname{gr}(\sigma)=\operatorname{gr}\left(\sigma^{\prime \prime}\right)$ and $\operatorname{dim} \sigma^{\prime}=$ $\operatorname{dim} \sigma^{\prime \prime}=\operatorname{dim} \sigma-1$ for which there is a gradient path $\gamma \in \Gamma_{A}\left(\sigma^{\prime}, \sigma^{\prime \prime}\right)$. In particular, the resolution is minimal if for all A-critical cells $\sigma$ and $\sigma^{\prime} \leq \sigma$, $\operatorname{dim} \sigma^{\prime}=\operatorname{dim} \sigma-1$ we have $\operatorname{gr}\left(\sigma^{\prime}\right) \neq \operatorname{gr}(\sigma)$.

Proof. The assertion follows from Theorem 3.4.2 together with Proposition 2.2.3. The second part of the corollary follows from the first together with the fact that gr is weakly decreasing along gradient paths. 


\section{Chapter 4}

\section{Applications}

This chapter consists of applications of Theorem 3.3.1 to produce cellular minimal $\mathbb{Z}^{d}$-graded free resolutions of certain classes of monomial modules and of the field $k$ over certain affine semigroup rings $k[\Lambda]$.

\subsection{Shellability}

In this section we construct free resolutions of co-Artinian monomial modules satisfying an additional property which we call shellability. We present an application of Theorem 3.3.1 to the $\mathrm{LCM}^{*}$-resolution (see Subsection 2.3.6) to obtain cellular free minimal resolutions for all shellable co-Artinian monomial modules. We will take advantage of the following proposition.

Proposition 4.1.1. [10] Let $X$ be a $C W$-complex, $P$ a poset.

Let

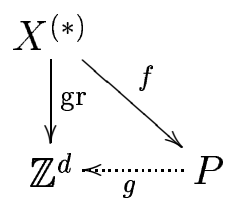

be a commutative diagram of poset maps such that $(X, \mathrm{gr})$ is a compactly $\left(\mathbb{Z}^{d}, \Lambda\right)$-graded $C W$-complex and $(X, f)$ a P-graded $C W$-complex. For each $p \in P$ let $A_{p}$ be an acyclic matching of the restriction of $G_{X}$ to $f^{-1}(p)$. Then $A=\bigcup_{p \in p} A_{p}$ is an acyclic homogeneous matching of $G_{X}$, in particular $H_{i}\left(\mathcal{C}_{X}^{\mathrm{gr}}\right) \cong H_{i}\left(\mathcal{C}_{X_{A}}^{\mathrm{gr}}\right)$ 
Proof. An argument independently found by Chari, Forman and Jonsson [10, Proposition 2.2] says that if the cells of the CW-complex $X$ are partitioned into sets $\left(B_{p}\right)_{p \in P}$ indexed by a partially ordered set $P$ such that $c^{\prime} \in B_{p^{\prime}}$, $c \in B_{p}$ and $c^{\prime} \leq c$ implies $p^{\prime} \leq p$ then any sequence $A_{p}, p \in P$, of acyclic matchings of the restriction of $G_{X}$ to $B_{p}, p \in P$, defines an acyclic matching $A=\bigcup_{p \in P} A_{p}$ of $G_{X}$. Now the result follows by Proposition 3.3.3.

Definition 4.1.2. A monomial module $M$ is called shellable if there is a total order "ㄷ" on $\operatorname{MinGen}(M)$ such that:

For $m, m^{\prime} \in \operatorname{MinGen}(M), m^{\prime} \sqsubset m$ there is an $m^{\prime \prime} \in \operatorname{MinGen}(M)$

$$
\begin{gathered}
\text { such that } m^{\prime \prime} \sqsubset m \text { and } \\
m \cdot x_{g\left(m, m^{\prime \prime}\right)}=\operatorname{lcm}\left(m^{\prime \prime}, m\right) \mid \operatorname{lcm}\left(m^{\prime}, m\right) \text { for some index } g\left(m, m^{\prime \prime}\right) .
\end{gathered}
$$

We list examples of shellable monomial modules. By " $\sqsubseteq$ revlex" we understand the reverse lexicographic order on monomials. Thus $x_{1}^{\alpha_{1}} \cdots x_{d}^{\alpha_{d}} \sqsubseteq_{\text {revlex }}$ $x_{1}^{\beta_{1}} \cdots x_{d}^{\beta_{d}}$ if and only if $\alpha_{d}=\beta_{d}, \ldots, \alpha_{i+1}=\beta_{i+1}$ and $\alpha_{i}>\beta_{i}$ for some $i \in[d]:=\{1, \ldots, d\}$.

- (Stable Modules) A monomial module $M$ is called stable if for any monomial $\underline{\mathbf{x}}^{\alpha} \in M, \alpha=\left(\alpha_{1}, \ldots, \alpha_{d}\right)$ and $j=\max \left\{i \mid \alpha_{i} \neq 0\right\}$ we have for all $l<j$ that $x_{l} \cdot \frac{\mathbf{x}^{\alpha}}{x_{j}} \in M$. We demonstrate that a shelling order for a stable monomial module is given by the order dual of "다lex". Assume $m=\underline{\mathbf{x}}^{\beta} \sqsubset_{\text {revlex }} m^{\prime}=\underline{\mathbf{x}}^{\alpha}$. If $i$ is the maximal index such that $x_{i}^{k} \mid m^{\prime}, x_{i}^{k} \not m$ for some $k \in \mathbb{N}$ and $j$ the maximal index such that $\alpha_{j} \neq 0$ then set $\bar{m}:=m / x_{j} \cdot x_{i}$. By stability $\bar{m} \in M$ and hence there is a monomial $m^{\prime \prime} \in \operatorname{MinGen}(M)$ such that $m^{\prime \prime} \mid \bar{m}$ and $\operatorname{lcm}\left(m, m^{\prime \prime}\right)=m \cdot x_{i} \mid \operatorname{lcm}\left(m, m^{\prime}\right)$. Clearly, $m \sqsubset_{\text {revlex }} m^{\prime \prime}$ and hence (4.1.4.1) follows. Moreover, if $M=M_{L}$ is given by a lattice $L$ then the order dual of " $\square_{\text {revlex" }}$ " is an $L$-equivariant shelling order for $M_{L}$. A minimal free resolution for stable ideals was first constructed by Eliahou and Kervaire [12]. 
- (Shellable Stanley-Reisner Ideals) If $\Delta$ is a simplicial complex on $[d]$ then the Stanley-Resiner ideal $I_{\Delta}$ of $\Delta$ is the monomial ideal whose generators are the monomials $\prod_{i \in A} x_{i}$ for the minimal non-faces $A$ of $\Delta$. Now if $\Delta^{*}=\{\sigma \subseteq[d] \mid[d] \backslash \sigma \notin \Delta\}$ is the combinatorial Alexanderdual of $\Delta$, then the minimal non-faces of $\Delta$ are the complements of the maximal faces of $\Delta^{*}$. Lateron in this section we discuss the concept of shellability for simplicial complexes. From the characterisation in Lemma 4.1.4 the reader will see that $\Delta^{*}$ is shellable if and only if $I_{\Delta}$ is shellable. See also Remark 4.1.7 for details on this subject.

- (Squarefree Stable Modules) A monomial ideal $M$ generated by squarefree monomials is called squarefree stable if for any monomial $\underline{\mathbf{x}}^{\alpha} \in M$ and $j=\max \left\{i \mid \alpha_{i} \neq 0\right\}$ we have for all $l<j$ with $x_{l} \mid \underline{\mathbf{x}}^{\alpha}$ that $x_{l} \cdot \frac{\underline{\mathbf{x}}^{\alpha}}{x_{j}} \in M$. Again the order dual of " $\sqsubseteq$ revlex" gives a shelling order satisfying (4.1.4.1). The proof is analogous to the stable case.

The concept of shellability is fundamental in the theory of simplicial complexes. Our definition of shellability for monomial modules can be viewed as a generalisation of this concept. In order to explain this and also in order to provide the tools that will be used to prove the main result in this section let us recall some of the theory of shellability of simplicial complexes as formulated in [6].

The dimension of a simplicial complex is defined to be the maximal dimension of its simplices. A simplicial complex $\Sigma$ is called pure if all maximal faces of $\Sigma$ have the same dimension. If $F$ is a face of $\Sigma$, we denote by $\bar{F}$ the simplicial complex consisting of all subsets of $\sigma$.

Definition 4.1.3. A simplicial complex $\Sigma$ is called shellable if there exists a total ordering $F_{1}, \ldots, F_{n}$ of its maximal faces such that for all $k=2, \ldots, n$

$$
\bar{F}_{k} \cap \bigcup_{i=1}^{k-1} \bar{F}_{i} \text { is a pure subcomplex of } \bar{F}_{k} \text { of dimension } \operatorname{dim} F_{k}-1 \text {. }
$$

An ordering $F_{1}, \ldots, F_{n}$ of the maximal faces of $\Sigma$ satisfying property 4.1.4.2 is called a shelling of $\Sigma$. 

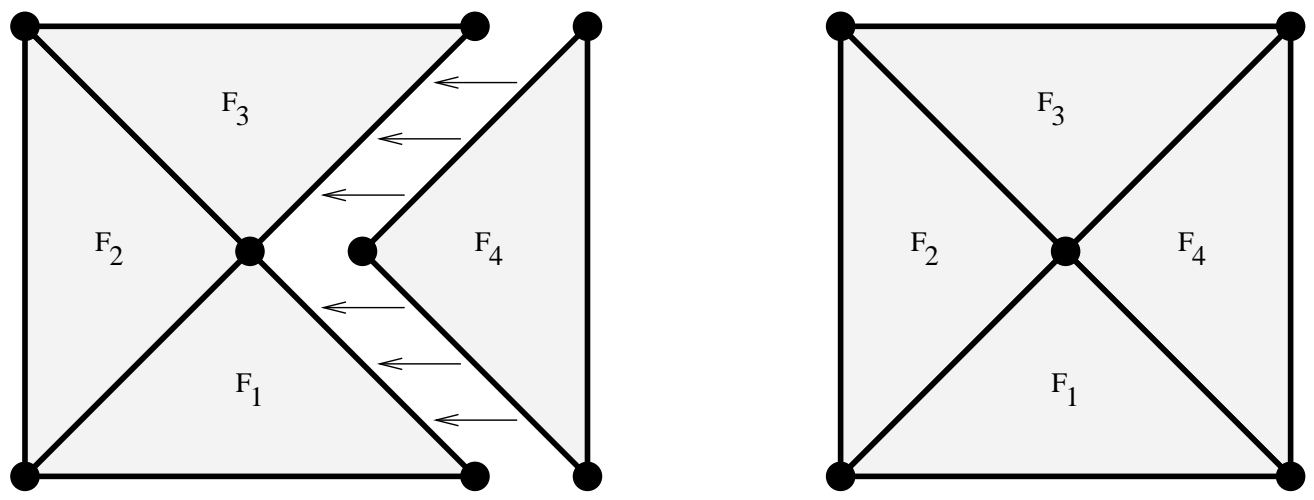

Figure 11. A shellable simplicial complex

with 4 maximal faces $F_{1}, F_{2}, F_{3}$ and $F_{4}$, all of dimension 2 .

The following Lemma gives an equivalent way to express shellability:

Lemma 4.1.4. A simplicial complex $\Sigma$ is shellable if and only if there exists a total ordering $F_{1}, \ldots, F_{n}$ of its maximal faces such that

$$
\begin{gathered}
\text { for } i, k \in\{1, \ldots, n\}, i<k \text { there is a } j \in\{1, \ldots, n\} \\
\text { such that } j<k \text { and } \\
F_{i} \cap F_{k} \subseteq F_{j} \cap F_{k}=F_{k}-\left\{x_{g(j, k)}\right\} \text { for some point } x_{g(j, k)} \in F_{k} .
\end{gathered}
$$

For an ordering $F_{1}, \ldots, F_{n}$ of the maximal faces of a simplicial complex $\Sigma$ we define the so-called restriction function $\mathcal{R}:\left\{F_{1}, \ldots, F_{n}\right\} \longrightarrow \Sigma$ by setting $\mathcal{R}\left(F_{k}\right)=\left\{x \in F_{k} \mid F_{k}-\{x\} \in \bigcup_{i=1}^{k-1} \bar{F}_{i}\right\}$. In terms of the restriction function $F_{1}, \ldots, F_{n}$ is a shelling of $\Sigma$ if and only if for all $k=2, \ldots, n$ we have $\bar{F}_{k} \cap \bigcup_{i=1}^{k-1} \bar{F}_{i}=\bigcup_{x \in \mathcal{R}\left(F_{k}\right)} \overline{F_{k}-\{x\}}$. Since $\bar{F}_{k}$ is the disjoint union of $\bigcup_{x \in \mathcal{R}\left(F_{k}\right)} \overline{F_{k}-\{x\}}$ and the intervall $\left[\mathcal{R}\left(F_{k}\right), F_{K}\right]$ of all subsets of $F_{k}$ that contain $\mathcal{R}\left(F_{k}\right)$ we also have:

Lemma 4.1.5. Let $\Sigma$ be a simplicial complex, $F_{1}, \ldots, F_{n}$ an ordering of its maximal faces with restriction map $\mathcal{R}$. Then $F_{1}, \ldots, F_{n}$ is a shelling of $\Sigma$ if and only if

$$
\Sigma=\bigcup_{k \in\{1, \ldots, n\}}\left[\mathcal{R}\left(F_{k}\right), F_{k}\right]
$$


We are now ready to present the following well known but crucial fact about shellable complexes:

Theorem 4.1.6. Let $\Sigma$ be a shellable finite simplicial complex. Then there exists an acyclic matching $A$ on $\Sigma$ such that the resulting Morse-complex $\Sigma_{A}$ is a wedge of spheres.

Proof. Every intervall $[A, B]=\{\sigma \in \Sigma \mid A \subset \sigma \subset B\}$ of simplices of $\Sigma$ such that $A \neq B$ and $A \neq \emptyset$ admits a perfect acyclic matching: Choose an element $x \in B-A$ and match according to the rule $\sigma \cup\{x\} \rightarrow \sigma-\{x\}$. With the characterisation of shellability according to Lemma 4.1 .5 we obtain a matching on $\Sigma$ where the critical cells are exactly those maximal faces $F$ such that

$$
\mathcal{R}(F)=F
$$

Therefore, the homotopy that transforms $\Sigma$ into its corresponding Morsecomplex consists of contracting everything to a single point except for the interiors of the faces satisfying 4.1.4.5. This proves the assertion.

Remark 4.1.7. Via Stanley-Reisner ideals, Definition 4.1 .2 can be viewed as a generalisation of the concept of shellability for simplicial complexes: For a simplicial complex $\Sigma$ on the vertex set $V=\left\{v_{1}, \ldots, v_{d}\right\}$ its Alexanderdual $\Sigma^{*}$ is defined to consist of all complements of sets $S \subset V$ such that $S \notin \Sigma$. Especially, the maximal faces of $\Sigma^{*}$ are the complements of the minimal non-faces of $\Sigma$. For a set $S \in \Sigma$ we denote by $x_{S} \in k\left[x_{1}, \ldots, x_{d}\right]$ the monomial $x_{S}:=\prod_{i \in S} x_{i}$. The Stanley-Reisner ideal $I_{\Sigma} \subset k\left[x_{1}, \ldots, x_{d}\right]$ is per definition generated by the monomials $x_{S}$ such that $S \notin \Sigma$. This implies that the correspondence $S \longmapsto x_{V-S}$ is a one-to-one-correspondence between $\operatorname{MinGen}\left(I_{\Sigma}\right)$ and the maximal faces of $\Sigma^{*}$. From Lemma 4.1.4 it is easily checked that for Stanley-Reisner ideals $I_{\Sigma}$ shellability of $\Sigma^{*}$ and shellability of $I_{\Sigma}$ are equivalent.

We obtain the following Theorem by applying Theorem 3.3.1 to the $\mathrm{LCM}^{*}$-resolution (see Subsection 2.3.6):

Theorem 4.1.8. Shellable co-Artinian monomial modules admit cellular minimal free resolutions. 
Recall (see Definition 2.3.26) that for a partially ordered set $P$ the order complex $\Delta(P)$ is defined to be the simplicial complex consisting of all finite chains in $P$, that is all finite subsets of $P$ on which the partial order of $P$ induces a total order. By means of this construction topological properties can be transferred to posets. For example, we say that a partially ordered set $P$ has the homotopy type of a wedge of spheres if this is true for $\Delta(P)$. Björner and Wachs introduce in [6] a theory for so-called lexicographical shellability of partially ordered sets, which makes it possible to read off shellablibilty of the order complex directly from the underlying poset. We review some of this theory here in order to provide the tools we need to prove Theorem 4.1.8.

Definition 4.1.9. A poset $P$ is called bounded if there exist elements $\hat{0}, \hat{1} \in$ $P$ such that $\hat{0}<x<\hat{1}$ for all $x \in P-\{\hat{0}, \hat{1}\}$. For a bounded poset $P$ we set $\bar{P}:=P-\{\hat{0}, \hat{1}\}$. For elements $x, y \in P, y$ is said to cover $x$ if $x<y$ and there is no $z \in P$ such that $x<z<y$. We also write $x \rightarrow y$ for this. An element $a \in P$ of a bounded poset $P$ is called atom if it covers $\hat{0}$. We use the following denotations:

$$
\begin{aligned}
\mathcal{E}(P) & :=\{(x, y) \in P \times P \mid x \rightarrow y\}, \\
\mathcal{M}(P) & :=\text { set of maximal chains in } P, \\
\mathcal{M E} E(P) & :=\{(m, x \rightarrow y) \in \mathcal{M}(P) \times \mathcal{E}(P) \mid x, y \in m\} .
\end{aligned}
$$

Definition 4.1.10. A chain-edge labeling of a poset $P$ is given by a map $\lambda: \mathcal{M} E(P) \rightarrow \Lambda$, with $\Lambda$ some poset, which satisfies:

$$
\begin{gathered}
\text { If } m, m^{\prime} \in \mathcal{M}(P), \\
m=\left\{x_{0}, \ldots, x_{s}\right\}, x_{i-1} \rightarrow x_{i} \text { for } i=1, \ldots, s, \\
m^{\prime}=\left\{x_{0}^{\prime}, \ldots, x_{t}^{\prime}\right\}, x_{i-1}^{\prime} \rightarrow x_{i}^{\prime} \text { for } i=1, \ldots, t, \\
\text { such that } x_{i}=x_{i}^{\prime} \text { for all } i=0, \ldots, d \\
\text { then } \lambda\left(m, x_{i-1} \rightarrow x_{i}\right)=\lambda\left(m^{\prime}, x_{i-1}^{\prime} \rightarrow x_{i}^{\prime}\right) \text { for all } i=1, \ldots, d .
\end{gathered}
$$

For elements $x, y \in P$ we denote by $[x, y]$ the interval $[x, y]:=\{z \in$ $P \mid x \leq z \leq y\}$. For a maximal chain $r$ in $[\hat{0}, x]$ we write $[x, y]_{r}$ for the so-called rooted intervall $[x, y]_{r}:=([x, y], r)$. Let $\Lambda^{*}$ be the set of all tuples 
of arbitrary length with entries in $\Lambda$. Consider the lexicographic partial order on $\Lambda^{*}$ defined by

$$
\left(x_{0}, \ldots, x_{s}\right) \leq_{L}\left(x_{0}^{\prime}, \ldots, x_{t}^{\prime}\right)
$$

if and only if

1. $x_{i}=x_{i}^{\prime}$ for all $i=0, \ldots, s$ and $s \leq t$ or

2. there exists $i \in\{1, \ldots, s\}$ such that $x_{j}=x_{j}^{\prime}$ for all $j<i$ and $x_{i}<x_{i}^{\prime}$.

Definition 4.1.11. Let $P$ be a bounded poset, $\Lambda$ a poset and $\lambda: \mathcal{M} E(P) \longrightarrow$ $\Lambda$ a chain-edge labeling. For every rooted interval $[x, y]_{r}$ of $P$ and every maximal chain $m=\left\{x_{0}, \ldots, x_{s}\right\}, x_{i-1} \rightarrow x_{i}, i=1, \ldots, s$ of the interval $[x, y]$ define

$$
\lambda_{r}(m):=\left(\lambda\left(m, x_{0} \rightarrow x_{1}\right), \ldots, \lambda\left(m, x_{s-1} \rightarrow x_{s}\right)\right) .
$$

$\lambda$ is called a CL-labeling (chain lexicographic labeling) if

1. for every rooted interval $[x, y]_{r}$ of $P$ there is a unique maximal chain $m=m_{[x, y]_{r}}=\left\{x_{0}, \ldots, x_{s}\right\}, x_{i-1} \rightarrow x_{i}, i=1, \ldots, s$ such that $\lambda\left(m, x_{0} \rightarrow\right.$ $\left.x_{1}\right)<\ldots<\lambda\left(m, x_{s-1} \rightarrow x_{s}\right)$ and

2. $\lambda_{r}\left(m_{[x, y]_{r}}\right)<_{L} \lambda_{r}\left(m^{\prime}\right)$ for all maximal chains $m^{\prime} \neq m_{[x, y]_{r}}$ of the interval $[x, y]$.

A bounded poset that admits a CL-labeling is called CL-shellable.

Björner and Wachs prove in [6] (Theorem 5.8) the following

Theorem 4.1.12. If a bounded poset $P$ is $C L$-shellable, then $\Delta(\bar{P})$ is shellable.

There is a different way to express CL-shellability which is more convenient for our needs:

Definition 4.1.13. A bounded poset $P$ is said to admit a recursive atom ordering if $P=\{\hat{0}, \hat{1}\}$ or if there is an ordering $a_{1}, \ldots, a_{n}$ of the atoms of $P$ such that: 
1. For all $i=1, \ldots, n$ the interval $\left[a_{i}, \hat{1}\right]$ admits a recursive atom ordering such that the atoms of $\left[a_{i}, \hat{1}\right]$ that belong to $\left[a_{j}, \hat{1}\right]$ for some $j<i$ come first.

2. For all $i<k$, if $a_{i}, a_{k}<y$ for some $y \in P$, then there exists a $j<k$ and an atom $z$ of $\left[a_{k}, \hat{1}\right]$ such that $a_{j}<z<y$.

Björner and Wachs prove in [6] (Theorem 5.11) the following

Theorem 4.1.14. A bounded poset $P$ admits a recursive atom ordering if and only if $P$ is $C L-$ shellable.

For our situation we get the following

Proposition 4.1.15. Let $M \subset k\left[x_{1}, \ldots, x_{d}\right]$ be a co-Artinian monomial module. We write $\mathrm{LCM}:=\operatorname{LCM}(\mathrm{M})$ and for $\alpha \in \mathbb{Z}^{d}$ we set $\mathrm{LCM}_{\leq \alpha}^{*}:=\{\sigma \in$ $\left.\mathrm{LCM}^{*} \mid \operatorname{lcm}(\sigma) \leq \alpha\right\}$. If $M$ is shellable then $\mathrm{LCM}_{\leq_{\alpha}}^{*} \cup\{1\}$ is CL-shellable for all $\alpha \in \mathbb{Z}^{d}$.

Proof. There is a unique maximal $\beta \in \mathbb{Z}^{d}$ such that $\beta \leq \alpha$ and $\underline{\mathbf{x}}^{\beta} \mid \operatorname{lcm}(\sigma)$ for some finite $\sigma \subset \operatorname{MinGen}(M)$. Therefore we have $\underline{\mathbf{x}}^{\beta} \in \mathrm{LCM}^{*}(\mathrm{M})$ and $\mathrm{LCM}_{\leq \alpha}^{*}=\mathrm{LCM}_{\leq \beta}^{*}$. Thus we can assume that $\underline{\mathbf{x}}^{\alpha} \in \mathrm{LCM}^{*}$.

Let $\alpha \in \mathbb{Z}^{d}$ such that $\underline{\mathbf{x}}^{\alpha} \in \mathrm{LCM}^{*}$, in particular this means that $\underline{\mathbf{x}}^{\alpha}$ is the unique maximal element in the bounded poset $\mathrm{LCM}_{\leq \alpha}^{*}$. Let $\sqsubseteq$ be an ordering on $\operatorname{Min} \operatorname{Gen}(M)$ satisfying condition 4.1.4.1. Let $\operatorname{Min} \operatorname{Gen}(M)_{\leq \alpha}:=$ $\{m \in \operatorname{MinGen}(M) \mid \operatorname{lcm}(m) \leq \alpha\}$.

As a first step we note that for all $m \in \mathrm{LCM}_{\leq \alpha}^{*}$ we have that any ordering of the atoms of the interval $\left[m, \underline{\mathbf{x}}^{\alpha}\right]$ is a recursive atom ordering of this interval. The proof proceeds by induction on the total degree of $\underline{x}^{\alpha} / m$ : If the total degree of $\underline{\mathbf{x}}^{\alpha} / m$ is 1 this means that $\left[m, \underline{\mathbf{x}}^{\alpha}\right]=\left\{m, \underline{\mathbf{x}}^{\alpha}\right\}$ and there is nothing to prove. Assume now that the assertion is correct for all intervals $\left[m^{\prime}, \underline{\mathbf{x}}^{\alpha}\right]$ such that the total degree of $\underline{\mathbf{x}}^{\alpha} / m^{\prime}$ is smaller than the total degree of $\underline{\mathbf{x}}^{\alpha} / m$. We need to verify conditions 1 and 2 from Definition 4.1.13. Condition 1 is fullfilled by induction hypothesis. Condition 2 is fullfilled because for atoms $a \neq b$ of the intervall $\left[m, \underline{\mathbf{x}}^{\alpha}\right]$ there are variables $x_{i}, x_{j}, i \neq j$ such that $a=x_{i} m, b=x_{j} m$, so that for any $y \in\left[m, \underline{\mathbf{x}}^{\alpha}\right]$ such that $a, b \mid y$ there is the atom $z:=x_{i} x_{j} m$ of $\left[b, \underline{\mathbf{x}}^{\alpha}\right]$ with $a \mid z$. 
As a second step we show that $\sqsubseteq$ defines a recursive atom ordering on $\operatorname{MinGen}(M)_{\leq \alpha}$ : Again we verify conditions 1 and 2 from Definition 4.1.13. Condition 1 is guaranteed by the first step of this proof. Condition 2 follows from property 4.1.4.1: For atoms $m, m^{\prime}$ of $\operatorname{LCM}_{\leq \alpha}^{*} \cup\{1\}$ with $m \sqsubset m^{\prime}$ and $y \in \mathrm{LCM}_{\leq \alpha}^{*}$ such that $m, m^{\prime} \mid y$ we have $\operatorname{lcm}\left(m, m^{\prime}\right) \mid y$ as well. So, by property 4.1.4.1 there exists a $m^{\prime \prime}$ in $\mathrm{LCM}^{*}$ such that $\operatorname{lcm}\left(m^{\prime \prime}, m\right)=$ $x_{g\left(m, m^{\prime \prime}\right)} m \mid \operatorname{lcm}\left(m, m^{\prime}\right)$. This implies that $m^{\prime \prime} \in \mathrm{LCM}^{*}(\mathrm{M})_{\leq \alpha}$ and that $z:=$ $x_{g\left(m, m^{\prime \prime}\right)} m$ is an atom of $\left[m, \underline{\mathbf{x}}^{\alpha}\right]$ with $m^{\prime \prime} \mid z$.

We are now ready to prove Theorem 4.1.8:

Proof. Let $M$ be a shellable co-Artinian monomial module. By Proposition 4.1.15 the poset $\mathrm{LCM}_{\leq \alpha}^{*} \cup\{1\}$ is CL-shellable for every $\alpha \in \mathbb{Z}^{d}$ and therefore, by Theorem 4.1 .12 , the ordercomplex $\Delta\left(\mathrm{LCM}_{<\alpha}^{*}\right)$ is shellable. Hence, by Theorem 4.1.6, there exists an acyclic Morse-matching $A_{\alpha}$ on $\Delta\left(\mathrm{LCM}_{<\alpha}^{*}\right)$, such that the resulting Morse-complex is a wedge of spheres. In particular, for all $i \in \mathbb{N}$ the number of critical cells in dimension $i$ in this matching is given by the Betti-number $\operatorname{dim} \tilde{H}_{i}\left(\Delta\left(\mathrm{LCM}_{<\alpha}^{*}\right)\right)$ of this complex.

Consider now the set of simplices $\sigma \in \Delta\left(\mathrm{LCM}_{\leq \alpha}^{*}\right)$ with grading $\operatorname{lcm}(\sigma)=$ $\alpha$, which we denote by $\Delta\left(\mathrm{LCM}_{\leq \alpha}^{*}\right)_{\alpha}$ : Since for $\sigma \in \Delta\left(\mathrm{LCM}_{\leq \alpha}^{*}\right)$ we have $\operatorname{lcm}(\sigma)=\alpha$ if and only if $\underline{\mathbf{x}}^{\alpha} \in \sigma$ we get:

$$
\Delta\left(\mathrm{LCM}_{\leq \alpha}^{*}\right)_{\alpha}=\left\{\sigma \dot{\cup}\left\{\underline{\mathbf{x}}^{\alpha}\right\} \mid \sigma \in \Delta\left(\mathrm{LCM}_{<\alpha}^{*}\right)\right\} .
$$

Therefore, for every $\alpha \in \mathbb{Z}^{d}$ the above matching $A_{\alpha}$ induces an acyclic matching on $\Delta\left(\mathrm{LCM}_{\leq \alpha}^{*}\right)_{\alpha}$ such that the number of critical cells in dimension $i$ is given by $\operatorname{dim} \tilde{H}_{i-1}\left(\Delta\left(\mathrm{LCM}_{<\alpha}^{*}\right)\right)$. Proposition 4.1 .1 shows that $A:=$ $\bigcup_{\alpha \in \mathbb{Z}^{d}} A_{\alpha}$ is an acyclic matching on $\Delta\left(\mathrm{LCM}^{*}\right)$. Since the $\mathbb{Z}^{d}$-graded simplicial complex supports a cellular resolution of $M$, we get, by Proposition 2.2.5, that the Betti-numbers of the minimal resolution for $M$ are also given by $\operatorname{dim} \tilde{H}_{i-1}\left(\Delta\left(\mathrm{LCM}_{<\alpha}^{*}\right)\right)$. This proves the assertion. 


\subsection{The Lyubeznik Resolution}

In this section we describe a general method to reduce the Taylor resolution of a monomial module by finding suitable homogeneous acyclic matchings of $X_{\text {Taylor }}$. The construction translates and generalizes an algebraic construction given by Lyubeznik [16] for monomial ideals.

If $f: X^{(*)} \rightarrow P$ is a map satisfying the assumptions of Proposition 4.1.1 then we call $f$ a gr-compatible $P$-grading of the $\left(\mathbb{Z}^{d}, \Lambda\right)$-graded $C W$-complex $X$.

Theorem 4.2.1. Let $M$ be a co-Artinian monomial $S$-module and $\left(X_{\text {Taylor }}, \mathrm{lcm}\right)$ the $\mathbb{Z}^{d}$-graded complex supporting the corresponding Taylor resolution. Let $P$ be a poset, $f$ a lcm-compatible $P$-grading of $X_{\text {Taylor }}$ and $\left(\preceq_{p}\right)_{p \in P}$ a sequence of total orderings of $\operatorname{MinGen}(M)$. Assume for all $p \in P$ and all finite $\sigma \subseteq \operatorname{MinGen}(M)$ such that $f(\sigma)=p, \sigma=\left\{m_{0} \prec_{p} \ldots \prec_{p} m_{i}\right\}$ the condition

$$
\begin{array}{cl}
m \mid \operatorname{lcm}\left(m_{t}, \ldots, m_{i}\right) \text { for some } & 0 \leq t<i, m \prec_{p} m_{t} \\
\Longrightarrow & f(\sigma \backslash\{m\})=f(\sigma \cup\{m\})=f(\sigma)=p .
\end{array}
$$

Then there exists a cellular resolution $\mathcal{F}_{X}^{\mathrm{lcm}}$ of $M$ supported by a $\mathbb{Z}^{d}$-graded $C W$-complex $X$, whose $i$-cells are indexed by all finite simplices $\sigma=\left\{m_{0} \prec_{f(\sigma)}\right.$ $\left.\ldots \prec_{f(\sigma)} m_{i}\right\} \subseteq \operatorname{MinGen}(M)$, such that

$$
m \nmid \operatorname{lcm}\left(m_{t}, \ldots, m_{i}\right) \text { for all } 0 \leq t<i \text { and } m \prec_{f(\sigma)} m_{t} \text {. }
$$

Proof. First, we note that if $M$ is a co-Artinian monomial module then the $\mathbb{Z}^{d}$-graded CW-complex $\left(X_{\text {Taylor }}, \mathrm{lcm}\right)$ is compactly graded.

For $\sigma=\left\{m_{0} \prec_{f(\sigma)} \ldots \prec_{f(\sigma)} m_{i}\right\}$ we define

$$
\nu(\sigma):=\sup \left\{\begin{array}{l|l}
k \in \mathbb{N} & \begin{array}{l}
\text { There exists } m \in \operatorname{MinGen}(M) \text { satisfying } \\
m \prec_{f(\sigma)} m_{i-k} \text { and } m \mid \operatorname{lcm}\left(m_{i-k}, \ldots, m_{i}\right)
\end{array}
\end{array}\right\},
$$

and if $\nu(\sigma) \neq-\infty$ let

$$
m(\sigma):=\min _{\prec_{f(\sigma)}}\left\{m \in \operatorname{MinGen}(M), \text { such that } m \mid \operatorname{lcm}\left(m_{i-\nu(\sigma)}, \ldots, m_{i}\right)\right\} .
$$


We claim that for each $p \in P$ the set of edges

$$
A_{p}:=\{\sigma \cup\{m(\sigma)\} \rightarrow \sigma \backslash\{m(\sigma)\} \mid f(\sigma)=p \text { and } \nu(\sigma) \neq-\infty\}
$$

defines an acyclic matching on the restriction of $G_{X_{\text {Taylor }}}$ to the set of vertices $f^{-1}(p)$. This follows by:

1. Condition (4.2.4.1) assures that all edges in $A_{p}$ are within the vertices $f^{-1}(p)$.

2. For any simplex $\sigma=\left\{m_{0}, \ldots, m_{i}\right\}$ and monomial $m$ such that $f(\sigma \cup$ $\{m\})=f(\sigma)=p$ we have

$$
\nu(\sigma \cup\{m\}) \geq \nu(\sigma) .
$$

Proof: If $\nu(\sigma)=-\infty$, there is nothing to prove. Thus we can assume

$$
m(\sigma) \mid \operatorname{lcm}\left(m_{i-\nu(\sigma)}, \ldots, m_{i}\right) .
$$

But this implies

$$
m(\sigma) \mid \operatorname{lcm}\left(m_{i-\nu(\sigma)}, \ldots, m_{i}, m\right)
$$

and we are done.

3. If $f(\sigma \cup\{m\})=f(\sigma)=p$ and $\nu(\sigma \cup\{m\})=\nu(\sigma) \neq-\infty$, then $m(\sigma \cup\{m\})=m(\sigma)$.

Proof: As seen in the proof of 2., we have $m(\sigma) \mid \operatorname{lcm}\left(m_{i-\nu(\sigma)}, \ldots, m_{i}, m\right)$. Hence for $\nu(\sigma \cup\{m\})=\nu(\sigma)$, we need $m \prec_{f(\sigma)} m_{i-\nu(\sigma)}$ and $m(\sigma \cup\{m\})$ to be the $\prec_{f(\sigma) \text {-minimal }}$ element of $\operatorname{MinGen}(M)$ dividing $\operatorname{lcm}\left(m_{i-\nu(\sigma)}, \ldots, m_{i}\right)$. This implies $m(\sigma \cup\{m\})=m(\sigma)$.

4. Whenever $\nu(\sigma) \neq-\infty$, we have

$$
\nu(\sigma \cup\{m(\sigma)\})=\nu(\sigma)=\nu(\sigma \backslash\{m(\sigma)\}) .
$$


Proof: If $m(\sigma) \in \sigma$, then

$$
\nu(\sigma \backslash\{m(\sigma)\}) \geq \nu(\sigma)
$$

follows from

$$
\left\{m_{i-\nu(\sigma)}, \ldots, m_{i}\right\} \subset \sigma \backslash\{m(\sigma)\} .
$$

If $m(\sigma) \notin \sigma, \nu(\sigma \cup\{m(\sigma)\}) \leq \nu(\sigma)$ follows from the fact, that for any $k \geq \nu(\sigma)$ and $m \prec_{f(\sigma)} m_{i-k}$, such that $m \mid \operatorname{lcm}\left(m_{i-k}, \ldots, m_{i}, m(\sigma)\right)$, we have $m \mid \operatorname{lcm}\left(m_{i-k}, \ldots, m_{i}\right)$, so $k=\nu(\sigma)$ and $m(\sigma) \preceq_{f(\sigma)} m$.

By 3. and 4 . the set of edges $A_{p}$ defines a matching. The facts 2 . and 4. imply that $\nu$ is constant on cycles, which in turn gives us acyclicity by the following argument: By 3., all elements $\tau$ of a cycle have the same $m(\tau)$. Thus $m(\tau)$ is the only element that possibly can be taken out in direction of the cycle. But taking it out again produces an arrow in direction opposite to the cycle. This proves that there cannot be any cycles at all.

By Proposition 4.1.1 the union $\bigcup_{p \in P} A_{p}$ defines an acyclic matching on $G_{X_{\text {Taylor }}}$. The critical cells $\sigma=\left\{m_{0} \prec_{f(\sigma)} \ldots \prec_{f(\sigma)} m_{i}\right\}$ of this matching are exactly those satisfying condition (4.2.4.2).

Definition 4.2.2. We call a cellular resolution that is constructed from the Taylor resolution using the method exhibited in Theorem 4.2.1 a Lyubeznik resolution.

Remark 4.2.3. Lyubeznik's original result from [16] is achieved when one takes a monomial ideal $M, P=\mathbb{Z}^{d}, f=\mathrm{lcm}$ and $\preceq_{p}$ a fixed linear order independent of $p$.

For applications to lattice ideals we need the following equivariant version of the Lyubeznik-resolution.

Definition 4.2.4. Let $L \subseteq \mathbb{Z}^{d}$ be an integer lattice such that $L \cap \mathbb{N}^{d}=\{\underline{0}\}$. Let $M_{L}$ be the monomial module spanned by $\left\{\underline{\mathbf{x}}^{\alpha} \mid \alpha \in L\right\}$.

1. We define an action

$$
L \times M_{L} \longrightarrow M_{L}
$$


by

$$
\left(\gamma, \underline{\mathbf{x}}^{\alpha}\right) \mapsto \gamma\left(\underline{\mathbf{x}}^{\alpha}\right):=\underline{\mathbf{x}}^{\alpha+\gamma}
$$

2. A poset $P$ is called $L$-poset if $L$ acts on $P$ as a group of order preserving automorphisms. Note that in our situation $L$ acts freely as a group of cellular homeomorphisms on $X_{\text {Taylor }}$ and $\mathbb{Z}^{d}$ is an $L$-poset.

3. Let $P$ and $Q$ be two $L$-posets. We call an order preserving map $f$ : $P \longrightarrow Q$ an L-equivariant poset map if $f$ respects the $L$-actions, that is, if for all $\gamma \in L$ and all $p \in P$, we have

$$
\gamma(f(p))=f(\gamma(p))
$$

4. For an $L$-poset $P$ we say that an $L$-equivariant poset map $f: X_{\text {Taylor }}^{(*)} \rightarrow$ $P$ is equivariantly lcm-compatible if there is a $L$-equivariant poset map $g: P \rightarrow \mathbb{Z}^{d}$ such that lcm $=g \circ f$.

Theorem 4.2.5. Let $L \subseteq \mathbb{Z}^{d}$ be a lattice such that $L \cap \mathbb{N}^{d}=\{\underline{\mathbf{0}}\}$. Let $\left(X_{\text {Taylor }}, \mathrm{lcm}\right)$ be the $\mathbb{Z}^{d}$-graded simplicial complex supporting the corresponding Taylor resolution of $M_{L}$. Let $P$ be an L-poset and let $f$ be an $L$ equivariantly lcm-compatible P-grading of $X_{\text {Taylor. }}$ Let $\left(\preceq_{p}\right)_{p \in P}$ be an Lequivariant sequence of total orderings of $\operatorname{MinGen}(M)$, that is: For $m, m^{\prime} \in$ $\operatorname{MinGen}(M)$ and $\gamma \in L$ we have $m \preceq_{p} m^{\prime} \Rightarrow \gamma(m) \preceq_{\gamma(p)} \gamma\left(m^{\prime}\right)$. Assume condition 4.2.4.1 for all finite $\sigma \subseteq \operatorname{MinGen}(M)$ such that $f(\sigma)=p$, $\sigma=\left\{m_{0} \prec_{p} \ldots \prec_{p} m_{i}\right\}$. Then the free action of $L$ on $X_{\text {Taylor }}$ induces a free action of $L$ on the $C W$-complex $X$ which supports the corresponding Lyubeznik-resolution $\mathcal{F}_{X}^{\mathrm{lcm}}$ of $M_{L}$.

Proof. The assumptions of $L$-equivariance of the poset map $f$ and the sequence $\left(\preceq_{p}\right)_{p \in P}$ assure that the matching $A$, as constructed in the proof of Theorem 4.2.1, as well as condition 4.2.4.2, are $L$-invariant. This proves the Theorem. Moreover, $X$ is $L$-equivariantly homotopy equivalent to $X_{\text {Taylor }}$. 


\subsubsection{Applications to Classes of Monomial Modules}

Proposition 4.2.6. For a generic co-Artinian monomial module $M$ there is a minimal free Lyubeznik-resolution. In particular, there exists a homogeneous acyclic matching $A$ of $X_{\text {Taylor }}$ for $X_{\text {Taylor }}$ such that $\left(X_{\text {Taylor }_{A}}, \mathrm{lcm}\right)$ $=\left(X_{\text {Scarf }}, \mathrm{lcm}\right)$. Moreover, $X_{\text {Scarf }}$ is a strong deformation retract of $X_{\text {Taylor }}$.

Proof. Choose $P=\left(\mathbb{Z}^{d}, \mathbb{N}^{d}\right)$ and $f=\mathrm{lcm}$ in the construction of Theorem 4.2.1. Let $\alpha=\left(\alpha_{1}, \ldots, \alpha_{d}\right) \in \mathbb{Z}^{d}$ be the lcm of some simplex from $X_{\text {Taylor. }}$ Let $\sigma_{1}, \ldots, \sigma_{k}$ be the minimal simplices of $X_{\text {Taylor }}$ such that $\operatorname{lcm} \sigma_{i}=\alpha$. Choose an ordering $\prec_{\alpha}$ on $\operatorname{MinGen}(M)$ such that the elements of $\Sigma_{\alpha}:=\sigma_{1} \cup \ldots \cup \sigma_{k}$ come last. Condition (4.2.4.1) of Theorem 4.2.1 is obviously satisfied. Consider the following cases:

1. If $k=1$, then there exists $\sigma \in \mathrm{lcm}^{-1}(\alpha)$ satisfying condition (4.2.4.2) of Theorem 4.2.1 if and only if $\operatorname{lcm}^{-1}(\alpha)=\left\{\sigma_{1}\right\}$.

2. Suppose $k>1$. Take any simplex $\sigma=\left\{m_{0} \prec_{\alpha} \ldots \prec_{\alpha} m_{q}\right\}$ with $\operatorname{lcm} \sigma=\alpha$. By minimality $\sigma$ contains at least one of the $\sigma_{i}$. Moreover there is an index $j$ such that $m_{l} \in \Sigma_{\alpha}$ if and only if $l \geq j$. Let $\sigma_{i^{\prime}} \neq \sigma_{i}$ be another minimal simplex with lcm equal to $\alpha$. There must be a variable $x_{r}$ and monomials $m \in \sigma_{i}, m^{\prime} \in \sigma_{i^{\prime}}$, such that $m \neq m^{\prime}$ and both $m$ and $m^{\prime}$ assume the exponent $\alpha_{r}$ on the variable $x_{r}$. Clearly, the binomial first syzygy corresponding to $m$ and $m^{\prime}$ is not generic. Now by $\left[5\right.$, Lemma 2.7] there is a monomial $\tilde{m}$ which divides $\operatorname{lcm}\left(m, m^{\prime}\right)$ in each variable properly. In particular, $\tilde{m}$ is not contained in $\Sigma_{\alpha}$, so $\tilde{m} \mid \operatorname{lcm}\left(m_{j}, \ldots, m_{q}\right)$ and condition (4.2.4.2) is violated. In particular, there exist no $\sigma \in \mathrm{lcm}^{-1}(\alpha)$ satisfying condition (4.2.4.2) of Theorem 4.2.1.

The construction of Theorem 4.2.1 yields a cellular resolution $\mathcal{F}_{X}^{\mathrm{lcm}}$ where $X$ equals the Scarf-complex $X_{\text {Scarf }}$. In particular, there is a homogeneous acyclic matching $A$ of $G_{X_{\text {Taylor }}}$ such that $\left(X_{\text {Taylor }_{A}}, 1 \mathrm{~cm}\right)=\left(X_{\text {Scarf }}, 1 \mathrm{~cm}\right)$. Since $A$ deletes an upper order ideal from $X_{\text {Taylor }}$ it follows by [13, Theorem 3.3] that $X_{\text {Scarf }}$ is a collaps of $X_{\text {Taylor }}$ and therefore a strong deformation retract.

For a lattice $L$ we denote by $I_{L}=\left\{\underline{\mathbf{x}}^{\alpha}-\underline{\mathbf{x}}^{\gamma} \mid \alpha-\gamma \in L\right\} \subseteq S$ the corresponding toric ideal. 
Corollary 4.2.7. [5, Example 3.12] Let $L \subseteq \mathbb{Z}^{d}$ be a lattice such that $L \cap$ $\mathbb{N}^{d}=\{\underline{\mathbf{0}}\}$. If $M_{L}$ is generic then $X_{\text {Scarf }} / L$ supports a minimal free cellular resolution of $I_{L}$.

Proof. By Proposition 4.2.6 the complex $X_{\text {Scarf }}$ supports a minimal free cellular resolution of $M_{L}$. It is simple to check that the linear orders $\preceq_{p}$ constructed in the proof of Proposition 4.2.6 can be chosen to fulfill the assumptions of Theorem 4.2.5. In particular, $X_{\text {Scarf }}$ is $L$-equivariantly homotopy equivalent to $X_{\text {Taylor }}$. Thus by [5, Corollary 3.7] $X_{\text {Scarf }} / L$ supports a minimal free resolution of $I_{L}$.

It was shown in Theorem 4.1.8 that for shellable co-Artinian monomial modules $M$ Theorem 3.3.1 can be applied to the $\mathrm{LCM}^{*}$-resolution of $M$ to produce a cellular minimal free resolution of $M$. We now present a second approach to this situation:

Proposition 4.2.8. Let $M$ be a shellable co-Artinian monomial module. Then there is a minimal free Lyubeznik-resolution for $M$.

Proof. Let $\sqsubseteq$ be a total order on $\operatorname{MinGen}(M)$ satisfying (4.1.4.1). For each multidegree $\alpha \in \operatorname{lcm}\left(X_{\text {Taylor }}^{(*)}\right) \subset \mathbb{Z}^{d}$, let $M_{\alpha}$ be the set of all $m \in \operatorname{MinGen}(M)$ such that there exists $\sigma \in \mathrm{lcm}^{-1}(\alpha)$ with $m \in \sigma$ being its maximal element with respect to $\sqsubseteq$. Let $P:=\left\{(\alpha, m) \mid \alpha \in \operatorname{lcm}\left(X_{\text {Taylor }}^{(*)}\right), m \in M_{\alpha}\right\}$, with partial order given by $(\alpha, m) \leq\left(\alpha^{\prime}, m^{\prime}\right) \Leftrightarrow \alpha \leq \alpha^{\prime}$ or $\left(\alpha=\alpha^{\prime}\right.$ and $\left.m \sqsubseteq m^{\prime}\right)$.

For $\sigma \in X_{\text {Taylor, }}$, we define $f(\sigma):=\left(\operatorname{lcm}(\sigma), \max _{\sqsubseteq}(\sigma)\right)$. For $m \in$ $\operatorname{MinGen}(M)$, there is a unique set $J_{m}$ consisting of all those indices $j$ such that there exists a monomial $n_{j}^{m} \in \operatorname{MinGen}(M)$ with $n_{j}^{m} \sqsubset m$ and $\operatorname{lcm}\left(n_{j}^{m}, m\right)=x_{j} m$. After choice of these $n_{j}^{m}$, we define for $p=(\alpha, m) \in P$ a total ordering $\prec_{p}$ on $\operatorname{MinGen}(M)$ by setting

- $N_{m}:=\left\{n_{j}^{m} \mid j \in J_{m}\right\}$,

- $N_{m} \prec_{p} \operatorname{MinGen}(M) \backslash N_{m}$,

- for $n_{j}^{m}, n_{j^{\prime}}^{m} \in N_{m}: n_{j}^{m} \prec_{p} n_{j^{\prime}}^{m} \Leftrightarrow j<j^{\prime}$,

- $\left.\prec_{p}\right|_{\operatorname{MinGen}(M) \backslash N_{m}}:=\left.\sqsubset\right|_{\operatorname{MinGen}(M) \backslash N_{m}}$. 
Condition (4.2.4.1) of Theorem 4.2.1 is satisfied. (Reason: Let $\sigma \in X_{\text {Taylor }}$ be such that $f(\sigma)=p, \sigma=\left\{m_{0} \prec_{p} \ldots \prec_{p} m_{i} \prec_{p} m\right\}$. If there are $0 \leq t \leq$ $i, \tilde{m} \prec_{p} m_{t}$, such that $\tilde{m} \mid \operatorname{lcm}\left(m_{t}, \ldots, m_{i}, m\right)$, we get $\tilde{m} \sqsubset m$, so $f(\sigma \cup\{\tilde{m}\})=$ $f(\sigma)=f(\sigma \backslash\{\tilde{m}\})$.)

We claim that the simplices $\sigma \in X_{\text {Taylor }}$ satisfying condition (4.2.4.2) of Theorem 4.2.1 are just those of the form $\sigma=L_{m} \cup\{m\}, L_{m} \subset N_{m}, p=$ $(\alpha, m) \in P$. Proof: Let $\sigma \in f^{-1}(\alpha, m), \tilde{m} \in \sigma, \tilde{m} \notin N_{m} \cup\{m\}$. We have $\tilde{m} \sqsubset m$, so there is by (4.1.4.1) a $m^{\prime \prime} \in \operatorname{MinGen}(M), m^{\prime \prime} \sqsubset m$, such that $\operatorname{lcm}\left(m^{\prime \prime}, m\right)=x_{j} m \mid \operatorname{lcm}(\tilde{m}, m)$ for some index $j$. We can choose $m^{\prime \prime}:=n_{j}^{m}$ and get: $n_{j}^{m} \prec_{p} \tilde{m}, n_{j}^{m} \mid \operatorname{lcm}(\tilde{m}, m)$, so $\sigma$ does not satisfy condition (4.2.4.2) of Theorem 4.2.1.

Now we verify minimiality by Corollary 3.4.5: Note that for all $p=$ $(\alpha, m) \in P$ the total degree $\operatorname{deg}(n)$ of $n$ is (weakly) smaller than $\operatorname{deg}(m)$ for all $n \in N_{m}$. Furthermore, for every $\sigma \in X_{\text {Taylor }}^{(*)}$ with $\operatorname{lcm}(\sigma)=\alpha, p=$ $(\alpha, m)$, we have, by the choice of $\prec_{p}$ (using notation of proof of Theorem 4.2.1): $\nu(\sigma) \neq-\infty \Rightarrow m(\sigma) \in N_{m}$. Let $\sigma=L_{m} \cup\{m\}, L_{m} \subset N_{m}$, be a simplex satisfying condition (4.2.4.2), and let $\sigma^{\prime}, \sigma^{\prime \prime}$ be such that $\operatorname{dim}\left(\sigma^{\prime}\right)=$ $\operatorname{dim}\left(\sigma^{\prime \prime}\right)=\operatorname{dim}(\sigma)-1, \sigma^{\prime}<\sigma, \operatorname{lcm}\left(\sigma^{\prime \prime}\right)=\operatorname{lcm}\left(\sigma^{\prime}\right)=\operatorname{lcm}(\sigma)$ and $\gamma$ a gradient path from $\sigma^{\prime}$ to $\sigma^{\prime \prime}$. We denote by $m^{\prime \prime}$ the $\sqsubset$-maximal element of $\sigma^{\prime \prime}=$ $L_{m^{\prime \prime}} \cup\left\{m^{\prime \prime}\right\}, L_{m^{\prime \prime}} \subset N_{m^{\prime \prime}}, p^{\prime \prime}=\left(\alpha, m^{\prime \prime}\right)$. We need to show that $\sigma^{\prime \prime}$ cannot satisfy condition (4.2.4.2).

The above implies that the total degree of all monomials of all simplices on $\gamma$ is (weakly) smaller than the total degree of $m$. In particular, $\operatorname{deg}\left(m^{\prime \prime}\right) \leq$ $\operatorname{deg}(m)$. We have: $\operatorname{dim} \sigma=\left|L_{m}\right|=\operatorname{deg}(\alpha)-\operatorname{deg}(m)$. If $\sigma^{\prime \prime}$ satisfies condition (4.2.4.2), we get $\operatorname{dim} \sigma^{\prime \prime}=\left|L_{m^{\prime \prime}}\right|=\operatorname{deg}(\alpha)-\operatorname{deg}\left(m^{\prime \prime}\right)$. But this contradicts $\operatorname{dim} \sigma^{\prime \prime}=\operatorname{dim} \sigma-1$.

Remark 4.2.9. For the Lyubeznik-resolution of a shellable co-Artinian monomial module $M$ as given in Proposition 4.2.8, $\sigma=L_{m} \cup\{m\}, L_{m}=$ $\left\{n_{j_{0}}, \ldots, n_{j_{t}}\right\} \subset N_{m}$ a critical simplex, $j_{0}<\ldots<j_{t}$, the differential $\partial e_{\sigma}$ is given by: 


$$
\begin{aligned}
& \left.\partial e_{\sigma}=\sum_{\nu=0}^{t}(-1)^{\nu} x_{j_{\nu}} e_{\sigma \backslash\left\{n_{j \nu}^{m}\right.}\right\}+(-1)^{t+1} . \\
& \sum_{\sigma^{\prime} \text { critical }} \sum_{\mathcal{P} \in \operatorname{GradPath}\left(\sigma \backslash\{m\}, \sigma^{\prime}\right)} \frac{\operatorname{lcm}(\sigma)}{\operatorname{lcm}\left(\sigma^{\prime}\right)} \cdot m(\mathcal{P}) e_{\sigma^{\prime}} . \\
& \operatorname{dim}\left(\sigma^{\prime}\right)=\operatorname{dim}(\sigma)-1
\end{aligned}
$$

Here $m(\mathcal{P})$ is defined as in Definition 3.4.1.

Proof. Considering the fact that the $\sigma \backslash\left\{n_{j_{\nu}}^{m}\right\}$ are critical the assertion follows from Theorem 3.4.2.

In Particular, we have the following corollaries.

Corollary 4.2.10. For a stable co-Artinian monomial module $M$ there is a minimal free Lyubeznik-resolution.

Corollary 4.2.11. Let $\Delta$ be a simplicial complex with shellable dual complex $\Delta^{*}$. Then there is a minimal free Lyubeznik-resolution for $I_{\Delta}$. In particular, there is a minimal free Lyubeznik-resolution for squarefree stable monomial ideals.

Proof. The existence of minimal free Lyubeznik-resolutions is a direct consequence of Proposition 4.2.8. The fact that squarefree stable monomial ideals are particular cases of Stanley-Reisner ideals of complexes with shellable dual can be found in [15].

Let $L$ be a lattice such that $M_{L}$ is co-Artinain and shellable. We call a linear order $\sqsubseteq$ on MinGen $\left(M_{L}\right)$ L-equivariant if $\underline{\mathbf{x}}^{\alpha^{\prime}} \sqsubseteq \underline{\mathbf{x}}^{\alpha}, \alpha, \alpha^{\prime} \in L$, implies $\underline{\mathbf{x}}^{\alpha^{\prime}+\gamma} \sqsubseteq \underline{\mathbf{x}}^{\alpha+\gamma}$ for $\gamma \in L$.

Corollary 4.2.12. Let $L \subseteq \mathbb{Z}^{d}$ be a lattice such that $L \cap \mathbb{N}^{d}=\{\underline{\mathbf{0}}\}$. If $M_{L}$ is shellable with an L-equivariant shelling order then there is a cellular complex $X_{\mathrm{SH}}$ on which $L$ acts vellularly and which supports a minimal free Lyubeznik resolution of $M_{L}$. Moreover, $X_{\mathrm{SH}} / L$ supports a minimal free cellular resolution of $I_{L}$. 
Proof. Analogous to the proof of Corollary 4.2.7. One has to use Proposition 4.2.8 and the same choices as made for the description of the differential above.

\subsection{Hypersimplex resolution of powers of the maximal homogeneous ideal}

In subsection 2.3.7 we presented a cellular $\mathbb{Z}^{d}$-graded free resolution of the powers $\mathfrak{m}^{n}$ of the maximal homogeneous ideal $\mathfrak{m}=\left(x_{1}, \ldots, x_{d}\right) \subset k\left[x_{1}, \ldots, x_{d}\right]$ which is supported by the polytopal complex $\mathcal{C}_{d}^{n}$. In this section we apply Theorem 3.3.1 to obtain cellular minimal $\mathbb{Z}^{d}$-graded free resolutions for $\mathfrak{m}^{n}$. We use the denotations from Definition 2.3.30.

Proposition 4.3.1. Matching $C_{\underline{a}, J} \rightarrow C_{\underline{a}+e_{\max J}, J \backslash\{\max J\}}$ for all $\underline{a} \in \mathbb{N}^{d}, J \subset$ $[d]$ such that $2 \leq n-|\underline{a}| \leq|J|-1$ and $\max J \geq \max \underline{a}:=\max \left\{i \in[d] \mid a_{i} \neq\right.$ $0\}$ yields an acyclic homogeneous discrete matching $A$ on $\mathcal{C}_{d}^{n}$. Here we set $\max \emptyset=0$. We denote by $\tilde{\mathcal{C}}_{d}^{n}:=\left(\mathcal{C}_{d}^{n}\right)_{A}$ the corresponding Morse complex. The A-critical cells of $\mathcal{C}^{n}$ are all the 0-cells $C_{\underline{a}, \emptyset}, \underline{a} \in \mathbb{N}^{d} \cap \Delta_{n}$ and all the cells $C_{\underline{a}, J}$ such that $\max J \geq \max \underline{a}$ and $|\underline{a}|=n-1$.

Proof. $A$ is a matching: Cells $C_{\underline{a}}, J$ on the left-hand side of a matching satisfy $\max J \geq \max a$, while cells $C_{\underline{a}^{\prime}, J^{\prime}}$ on the right-hand side satisfy $\max J^{\prime}<$ $\max \underline{a}^{\prime}$.

Acyclicity of $A$ : On cycles, $\operatorname{lcm}\left(C_{\underline{a}, J}\right)$ is decreasing, so it must be constant. This implies that max $J$ is constant on cycles which is a contradiction to the existence of nontrivial cycles.

Proposition 4.3.2. Denoting by $\tilde{\partial}$ the differential of the Morse complex $\tilde{\mathcal{C}}_{d}^{n}:=\left(\mathcal{C}_{d}^{n}\right)_{A}$ and by $\tilde{C}_{\underline{a}, J}$ the cell in this Morse complex corresponding to the A-critical cell $C_{\underline{a}}, J$ of $\mathcal{C}_{d}^{n}$, we have for $J=\left\{j_{0}, \ldots, j_{r}\right\},|J| \geq 3$ :

If $\max \underline{a} \leq \max (J \backslash\{\max J\})$, then

$$
\tilde{\partial} \tilde{C}_{\underline{a}, J}=\sum_{\nu=0}^{r}(-1)^{\nu} \tilde{C}_{\underline{a}, J_{\nu}} .
$$


If $\max \underline{a}>\max (J \backslash\{\max J\})$, then

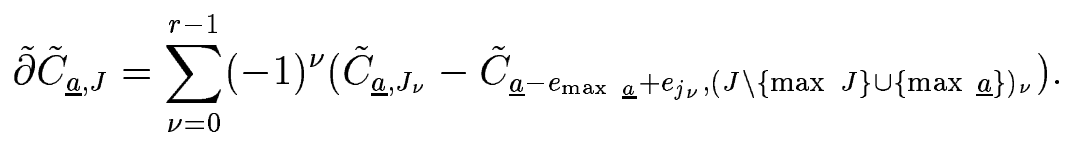

For $J=\left\{j_{0}, j_{1}\right\}$ we have, as in $\mathcal{C}_{d}^{n}$

$$
\tilde{\partial} \tilde{C}_{\underline{a},\left\{j_{0}, j_{1}\right\}}=\tilde{C}_{\underline{a}+e_{j_{1}}, \emptyset}-\tilde{C}_{\underline{a}+e_{j_{0}}, \emptyset}
$$

Proof. Let $C_{\underline{a}}, J$ be $A$-critical, (so $\max J \geq \max \underline{a}$ ), $J=\left\{j_{0}, \ldots, j_{r}\right\}, r \geq 2$. For $\nu=0, \ldots, r-1$, the cells $C_{\underline{a}, J_{\nu}}$ are critical, and if $\max \underline{a} \leq \max J_{r}$,

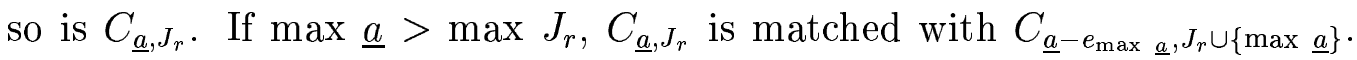

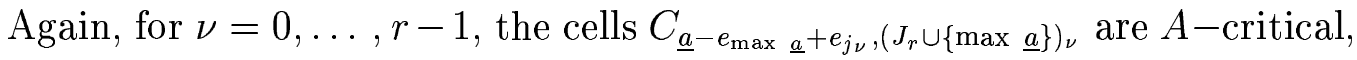

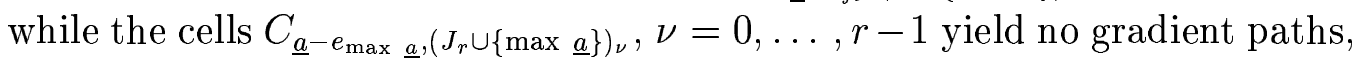
because they are matched "down" with the cells $C_{\underline{a}},\left(J_{r}\right)_{\nu}$. Finally, $C_{\underline{a}-e_{\max } \underline{a}, J_{r}}$ also yields no gradient paths, because $\left|\underline{a}-e_{\max \underline{a}}\right|=n-2$, which leaves no possibility for a gradient path to a critical cell $\left.C_{\underline{a}^{\prime}, J^{\prime}},\left|\underline{a}^{\prime}\right|=n-1\right)$, while, if $\max J_{r}=j_{r-1} \geq \max \left(\underline{a}-e_{\max } \underline{a}\right)$, it is matched 'down' with $C_{\underline{a}}-e_{\max } \underline{a}+e_{j_{r-1}},\left(J_{r}\right)_{r-1}$. Considering orientations proves the assertion.

Proposition 4.3.3. $\tilde{C}_{d}^{n}$ defines a multigraded cellular minimal free resolution of $\mathfrak{m}^{n}$.

Proof. Corollary 3.4.5 together with Proposition 4.3.2 proves the assertion. 


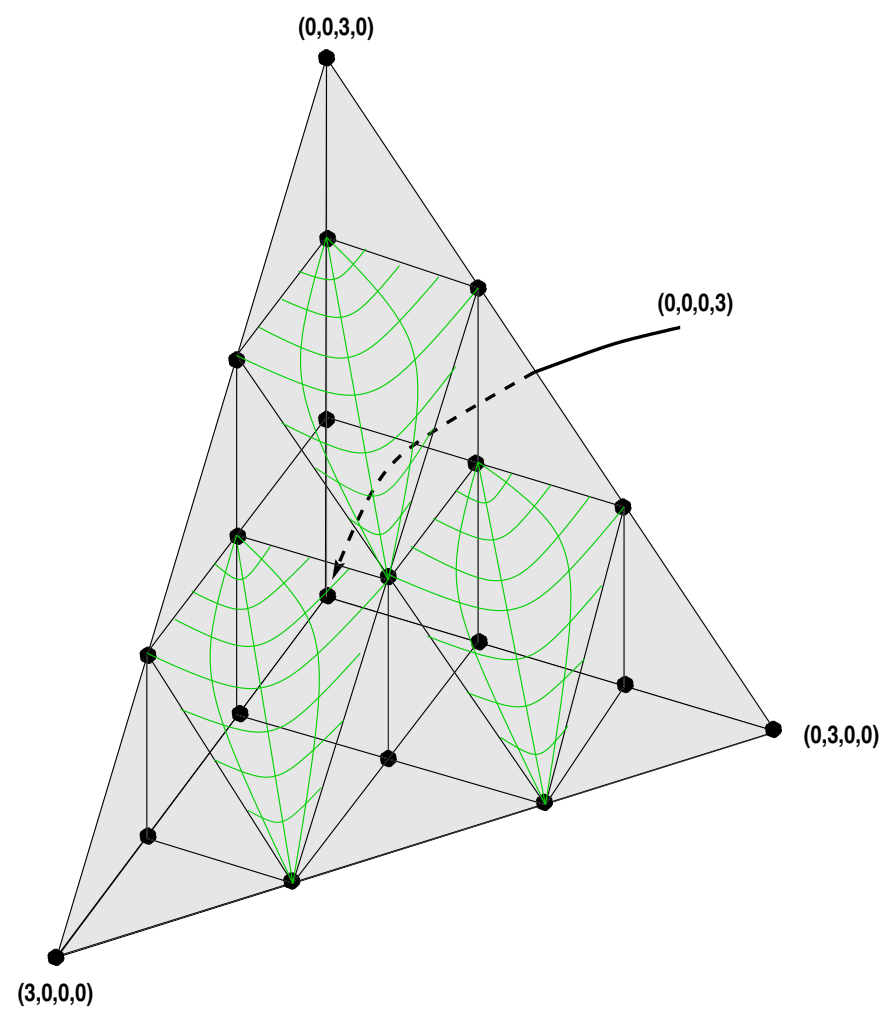

Figure 12. The complex $\tilde{\mathcal{C}}_{4}^{3}$

Remark 4.3.4. The multigraded cellular minimal free resolution of $\mathfrak{m}^{n}$ defined by $\tilde{C}_{d}^{n}$ is isomorphic to the one resulting from the Lyubeznik-construction (see Corollary 4.2.10). Both are isomorphic to the one given by Eliahou and Kervaire [12].

Proof. We establish a one-to-one-correspondence between the cells of the two Morse complexes by mapping the Lyubeznik-critical cell

$$
\sigma=\left\{n_{j_{0}}^{m}, \ldots, n_{j_{r}}^{m}, m\right\}=\left\{x_{j_{0}} \frac{m}{x_{\max m}}, \ldots, x_{j_{r}} \frac{m}{x_{\max m}}, m\right\}
$$

of the Taylor-complex $X_{\text {Taylor }}$ (that is $\alpha=\operatorname{deg} m+\sum_{\nu=0}^{r} e_{j_{\nu}}$. For the definition of $n_{j_{\nu}}^{m}$ see proof of Proposition 4.2.8,) to the $A$-critical cell $C_{\underline{a}^{\prime}, J^{\prime}}$ of $\mathcal{C}_{n}^{d}$, with $\underline{a}^{\prime}:=\operatorname{deg}\left(\frac{m}{x_{\max m}}\right), J^{\prime}:=\left\{j_{0}, \ldots, j_{r}, \max m\right\}$ (of course sending the $0-$ cells $\sigma=\{m\}$ to the 0 -cells $C_{\underline{a}, \emptyset}$, where $\underline{a}=\operatorname{deg} m$ ). Analysing the gradient paths 
of the Lyubeznik-matching shows that the Lyubeznik-differential is given by:

$$
\begin{aligned}
\partial\left\{n_{j_{0}}^{m}, \ldots, n_{j_{r}}^{m}, m\right\}= & \sum_{\nu=0}^{r}(-1)^{\nu}\left\{n_{j_{0}}^{m}, \ldots, \widehat{n_{j_{\nu}}^{m}}, \ldots, n_{j_{r}}^{m}, m\right\} \\
& +(-1)^{r+1}\left\{n_{j_{0}}^{m}, \ldots, n_{j_{r}}^{m}\right\}
\end{aligned}
$$

if $t:=\max a^{\prime}=\max \left(\frac{m}{x_{\max m}}\right) \leq j_{r}$, and

$$
\begin{aligned}
\partial\left\{n_{j_{0}}^{m}, \ldots, n_{j_{r}}^{m}, m\right\}=\sum_{\nu=0}^{r} & (-1)^{\nu}\left(\left\{n_{j_{0}}^{m}, \ldots, \widehat{n_{j_{\nu}}^{m}}, \ldots, n_{j_{r}}^{m}, m\right\}\right. \\
& \left.\quad-\left\{n_{j_{0}}^{n_{j_{\nu}}^{m}}, \ldots, \widehat{n_{j_{\nu}}^{n_{j_{\nu}}^{m}}}, \ldots, n_{j_{j_{\nu}}}^{n_{j_{\nu}}^{m}}, n_{j_{\nu}}^{m}\right\}\right),
\end{aligned}
$$

where $\alpha^{\prime}=\alpha-e_{\max m}$, if $t>j_{r}$. Proof: Let $\sigma=\left\{n_{j_{0}}^{m}, \ldots, n_{j_{r}}^{m}, m\right\}$ be a Lyubeznik-critical cell, $r \geq 1$. The cells $\sigma_{\nu}=\left\{n_{j_{0}}^{m}, \ldots, \widehat{n_{j_{\nu}}^{m}}, \ldots, n_{j_{r}}^{m}, m\right\}$, $\nu=0, \ldots, r$ are critical. $\tilde{\sigma}=\left\{n_{j_{0}}^{m}, \ldots, n_{j_{r}}^{m}\right\}$ is critical if and only if

$$
t:=\max \left(\frac{m}{x_{\max m}}\right) \leq j_{r}
$$

which is the translation of the condition $\max \underline{a} \leq \max (J \backslash\{\max J\})$ (see 4.3.4.1) under the given one-to-one-correspondence. This proves equality of the differentials if this condition is satisfied. In what follows we suppose that condition 4.3.4.3 is violated. We use the following notations:

- $t:=\max \left(\frac{m}{x_{\max m}}\right)$

- $n\left(j_{\nu}, j_{\nu^{\prime}}\right)=n\left(j_{\nu^{\prime}}, j_{\nu}\right):=x_{j_{\nu}} x_{j_{\nu^{\prime}}} \frac{m}{x_{t} x_{\max m}}=n_{j_{\nu^{\prime}}}^{n_{j_{\nu}}^{m}}=n_{j_{\nu}}^{n_{j^{\prime}}^{m}}$, for $0 \leq \nu<$ $\nu^{\prime} \leq r$

- $\sigma_{k, l}^{i}:=\left\{\begin{array}{c}n\left(j_{0}, j_{l}\right), \ldots, n\left(j_{i-1}, j_{l}\right), n\left(j_{i}, j_{l+1}\right), \ldots \\ \ldots, n\left(j_{k}, j_{l+1}\right), \ldots, n\left(j_{k}, j_{r}\right), n_{j_{k}}, \ldots, n_{j_{l}}\end{array}\right\}$ and $\tau_{k, l}^{i}:=\sigma_{k, l}^{i} \cup\left\{n\left(j_{i}, j_{l}\right)\right\}$ for $0 \leq i \leq k \leq l<r$ and for $0 \leq i=k \leq l=r$. (Undefined symbols $n\left(j_{\nu}, j_{r+1}\right)$ are to be omitted, $\tau_{k, l}^{i}$ only defined for $i \neq l$.) 
Consider the following gradient paths:

- $Q_{k}:=\sigma_{0, r}^{0} \rightarrow \tau_{0, r}^{0} \rightarrow \sigma_{1, r}^{1} \rightarrow \tau_{1, r}^{1} \rightarrow \ldots \rightarrow \tau_{k-1, r}^{k-1} \rightarrow \sigma_{k, r}^{k}, k=0, \ldots, r$;

- $R_{k, l}:=\sigma_{k, l+1}^{k} \rightarrow \tau_{k, l+1}^{k} \rightarrow \sigma_{k, l}^{0} \rightarrow \tau_{k, l}^{0} \rightarrow \ldots \rightarrow \tau_{k, r}^{k-1} \rightarrow \sigma_{k, l}^{k}, 0 \leq k \leq l<$ $r$.

We claim that there exist exactly the gradient paths

$$
P_{k}:=R_{k, k} \circ R_{k, k+1} \circ \ldots \circ R_{k, r-1} \circ Q_{k}
$$

from $\sigma_{0, r}^{0}$ to a Lyubeznik-critical cell of same dimension. (When $P=\sigma_{1} \rightarrow \ldots \rightarrow \sigma_{s}, \quad Q=\sigma_{s} \rightarrow \ldots \rightarrow \sigma_{s+t}$, we denote $Q \circ P:=\sigma_{1} \rightarrow$ $\left.\ldots \rightarrow \sigma_{s+t}.\right)$

Since the $P_{k}$ start at $\sigma_{0, r}^{0}=\tilde{\sigma}$ and end at $\sigma_{k, k}^{k}=\left\{n\left(j_{0}, j_{k}\right), \ldots, n\left(j_{k-1}, j_{k}\right)\right.$, $\left.n\left(j_{k}, j_{k+1}\right), \ldots, n\left(j_{k}, j_{r}\right), n_{j_{k}}\right\}$, considering orientations proves the assertion. Proof of the claim:

For all $0 \leq i \leq k \leq l<r$ and all $0 \leq i=k \leq l=r$ such that $i \neq l$, there is a matching

$$
\sigma_{k, l}^{i} \leftarrow \tau_{k, l}^{i}
$$

because in $\sigma_{k, l}^{i}=\left\{\begin{array}{c}n\left(j_{0}, j_{l}\right), \ldots, n\left(j_{i-1}, j_{l}\right), n\left(j_{i}, j_{l+1}\right), \ldots \\ \ldots, n\left(j_{k}, j_{l+1}\right), \ldots, n\left(j_{k}, j_{r}\right), n_{j_{k}}, \ldots, n_{j_{l}}\end{array}\right\}$, the maximal 'tail' (see proof of Theorem 4.2.1) whose lcm is devided by a $\prec_{\left(\alpha^{\prime}, n_{j_{l}}\right)^{-}}$ smaller element is $\left\{n\left(j_{i}, j_{l+1}\right), \ldots, n\left(j_{k}, j_{l+1}\right), \ldots, n\left(j_{k}, j_{r}\right), n_{j_{k}}, \ldots, n_{j_{l}}\right\}$ and is $\prec_{\left(\alpha^{\prime}, n_{j_{l}}\right)}$-minimaly devided by $n\left(j_{i}, j_{l}\right)$.

1. For $0 \leq i=k<l=r$, we have

$$
\tau_{k, l}^{i}=\tau_{k, r}^{k}=\left\{n\left(j_{0}, j_{r}\right), \ldots, n\left(j_{k}, j_{r}\right), n_{j_{k}}, \ldots, n_{j_{r}}\right\}
$$

The next matching will be taking out again $n\left(j_{k}, j_{r}\right)$, (which won't give us a gradient path to a critical cell, because these always alternate in dimension between the dimensions of $\tilde{\sigma}$ and $\sigma$ ) unless we at once get rid of either $n_{j_{k}}$ or $n_{j_{r}}$ : 
- $\tau_{k, r}^{k} \backslash\left\{n_{j_{k}}\right\}=\sigma_{k+1, r}^{k+1}=\left\{n\left(j_{0}, j_{r}\right), \ldots, n\left(j_{k}, j_{r}\right), n_{j_{k+1}}, \ldots, n_{j_{r}}\right\}$,

- $\tau_{k, r}^{k} \backslash\left\{n_{j_{r}}\right\}=\sigma_{k, r-1}^{0}=\left\{n\left(j_{0}, j_{r}\right), \ldots, n\left(j_{k}, j_{r}\right), n_{j_{k}}, \ldots, n_{j_{r-1}}\right\}$.

These two possibilities represent the possibilities to either go on in some $Q_{\tilde{k}}, \tilde{k}>k$, or to switch over to $R_{k, r-1}$.

2. For $0 \leq i \leq k \leq l<r, i \neq l$, we have

$$
\tau_{k, l}^{i}=\left\{\begin{array}{c}
n\left(j_{0}, j_{l}\right), \ldots, n\left(j_{i}, j_{l}\right), n\left(j_{i}, j_{l+1}\right), \ldots \\
\ldots, n\left(j_{k}, j_{l+1}\right), \ldots, n\left(j_{k}, j_{r}\right), n_{j_{k}}, \ldots, n_{j_{l}}
\end{array}\right\} .
$$

Here the next matching will be taking out again $n\left(j_{i}, j_{l}\right)$, unless we at once get rid of either $n_{j_{l}}$ or $n\left(j_{i}, j_{l+1}\right)$.

(a) If $i<k$, we cannot take out $n_{j_{l}}$, because eventually (grin!), we would be forced to take out all $n_{j_{\nu}}$, but: Since, for a critical cell $\tau$, we have $\operatorname{dim} \tau=\operatorname{deg} \tau-\operatorname{deg}(\max \tau)$, and since in the case of $m_{n}^{d}$ all monomials have the same degree, $\operatorname{deg} \tau$ must be constant on gradient paths, so at least one of the $n_{j_{\nu}}$ must stay. So we take out $n\left(j_{i}, j_{l+1}\right)$, which leaves us with

$$
\sigma_{k, l}^{i+1}=\tau_{k, l}^{i} \backslash\left\{n\left(j_{i}, j_{l+1}\right)\right\}=\left\{\begin{array}{l}
n\left(j_{0}, j_{l}\right), \ldots, n\left(j_{i}, j_{l}\right), \\
n\left(j_{i+1}, j_{l+1}\right), \ldots, n\left(j_{k}, j_{l+1}\right), \ldots \\
\ldots, n\left(j_{k}, j_{r}\right), n_{j_{k}}, \ldots, n_{j_{l}}
\end{array}\right\}
$$

(b) If $i=k<l$, we cannot take out $n\left(j_{i}, j_{l+1}\right)$, because with $n_{j_{i}}=$ $n_{j_{k}} \in \tau_{k, l}^{i}$, the next matching would still be taking out $n\left(j_{i}, j_{l}\right)$. So we take out $n_{j_{l}}$, which leaves us with

$$
\sigma_{k, l-1}^{0}=\tau_{k, l}^{k} \backslash\left\{n_{j_{l}}\right\}=\left\{\begin{array}{l}
n\left(j_{0}, j_{l}\right), \ldots, n\left(j_{k}, j_{l}\right), \ldots, \\
\ldots, n\left(j_{k}, j_{r}\right), n_{j_{k}}, \ldots, n_{j_{l-1}}
\end{array}\right\} .
$$

This proves existence and uniqueness of the given gradient paths, as claimed.

Since, if $t>j_{r}$, the above correspondence takes

$$
\left\{n_{j_{0}}^{n_{j_{\nu}}^{m}}, \ldots, \widehat{n_{j_{\nu}}^{n_{j_{\nu}}^{m}}}, \ldots, n_{j_{\nu}}^{n_{j_{\nu}}^{m}}, n_{j_{\nu}}^{m}\right\}
$$


to $C_{\underline{a}^{\prime}-e_{t}+e_{j_{\nu}},\left\{j_{0}, \ldots, \hat{j_{\nu}}, \ldots, j_{r}, t\right\}}$, it follows that it also identifies the two differentials. On the other hand, by mapping $\left\{n_{j_{0}}^{m}, \ldots, n_{j_{r}}^{m}, m\right\}$ to $e\left(j_{0}, \ldots, j_{r} ; m\right)$ there is a one-to-one-correspondence between the Lyubeznik-critcal cells and the admissible symbols of Eliahou and Kervaire [12], which identifies the differentials up to a sign (up to choice of orientations, respectively).

\subsection{Resolutions via rooted complexes revis- ited}

In this section we show that there exists a suitable matching for the Taylor complex so that Theorem 3.3.1 recovers the cellular free resolution via rooted complexes as presented in Section 2.3.5 (see 2.3.5 for definitions concerning the rooted complex).

Proposition 4.4.1. Let I be a monomial ideal,

$$
\pi: \operatorname{LCM}(\mathrm{I}) \longrightarrow \operatorname{MinGen}(\mathrm{I})
$$

a rooting map on its LCM-lattice. Then there exists an acyclic homogeneous matching $A$ on $X_{\text {Taylor }}$ such that $X_{A}=\mathcal{R} C(I, \pi)$.

We need the following Lemma:

Lemma 4.4.2. Let $B$ and $B^{\prime}$ be broken subsets of $\operatorname{MinGen}(I)$. Then $B \cup B^{\prime}$ is broken as well.

Proof. Since $B$ and $B^{\prime}$ are broken, we have: $\tilde{\pi}(B) \notin B$ and $\tilde{\pi}\left(B^{\prime}\right) \notin B^{\prime}$. Assume $\tilde{\pi}\left(B \cup B^{\prime}\right) \in B \cup B^{\prime}$, say $\tilde{\pi}\left(B \cup B^{\prime}\right) \in B$. This implies $\pi(\operatorname{lcm}(B \cup$ $\left.\left.B^{\prime}\right)\right)|\operatorname{lcm} B| \operatorname{lcm}\left(B \cup B^{\prime}\right)$ which again implies $\pi(\operatorname{lcm}(B))=\pi\left(\operatorname{lcm}\left(B \cup B^{\prime}\right)\right)$ by the properties of Definition 1. But this is a contradiction to $B$ being broken.

Now we prove Proposition 4.4.1:

Proof. By Lemma 4.4.2, for every simplex $\sigma \in X_{\text {Taylor, }}$, there is a maximal broken subset $B_{\sigma} \subset \sigma$. We define the following matching on $G_{X_{\text {Taylor }}}$ :

$$
\sigma \cup\left\{\tilde{\pi}\left(B_{\sigma}\right)\right\} \rightarrow \sigma-\left\{\tilde{\pi}\left(B_{\sigma}\right)\right\} .
$$


To see that $A$ is well defined we must check that

1. if $\tilde{\pi}\left(B_{\sigma}\right) \in \sigma$ then $B_{\sigma-\left\{\tilde{\pi}\left(B_{\sigma}\right)\right\}}=B_{\sigma}$ and

2. if $\tilde{\pi}\left(B_{\sigma}\right) \notin \sigma$ then $B_{\sigma \cup\left\{\tilde{\pi}\left(B_{\sigma}\right)\right\}}=B_{\sigma}$.

The first assertion follows from the fact that $\tilde{\pi}\left(B_{\sigma}\right) \notin B_{\sigma}$, the second one from the fact that $B_{\sigma} \cup\left\{\tilde{\pi}\left(B_{\sigma}\right)\right\}$ is not broken. Condition 4.4.4.1 implies that $A$ is a matching. Acyclicity follows from the fact that along directed paths in $\left(G_{X_{\text {Taylor }}}\right)_{A}$ the maximal broken subset decreases. Therefore, Theorem 3.3.1, yields a Morse complex $\left(X_{\text {Taylor }}\right)_{A}$ consisting of cells that are in one-to-one correspondence to those cells of $X_{\text {Taylor }}$ that dont contain a broken subset, that is those cells of $X_{\text {Taylor }}$ that are rooted. Beyond that, $\left(X_{\text {Taylor }}\right)_{A}$ emerges from $X_{\text {Taylor }}$ by elementary collapses of maximal simplices, so therefore we have $X_{A}=\mathcal{R} C(I, \pi)$.

\section{5 p-Borel fixed ideals}

In this section we study monomial ideals that are $p$-Borel fixed. We obtain cellular minimal resolutions for a special subclass, namely the class of monomial ideals that are principal p-Borel fixed such that the generator (generator in a sense defined below) is a power of one single variable. Here are the definitions:

Definition 4.5.1. Let $p \in \mathbb{N}$ be a prime. Define the partial order $\leq_{p}$ on $\mathbb{N}$ by setting $a \leq_{p} b$ if and only if the $p$-adic expansions $a=\sum_{i=0}^{s} a_{i} p^{i}$ and $b=\sum_{i=0}^{s} b_{i} p^{i}$ fullfill $a_{i} \leq b_{i}$ for all $i=0, \ldots, s$. A monomial ideal $I \subset k\left[x_{1}, \ldots, x_{d}\right]$ is called p-Borel fixed if for all monomials $m \in I$ and all variables $x_{i} \in k\left[x_{1}, \ldots, x_{d}\right]$ we have

$$
x_{i}^{l}\left|m, x_{i}^{l+1}\right|\left\{m, j<i \text { and } k \leq_{p} l \Rightarrow\left(x_{j} / x_{i}\right)^{k} m \in I\right.
$$

It is known (see [1]) that this property only needs to be checked for the set $\operatorname{MinGen}(I)$ of minimal generators of $I$ :

Lemma 4.5.2. Let $I \subset k\left[x_{1}, \ldots, x_{d}\right]$ be a monomial ideal. Then the following conditions are equivalent: 


\section{I is p-Borel fixed}

2. Condition 4.5.4.1 is fullfilled for all minimal generators $m \in \operatorname{MinGen}(I)$.

Definition 4.5.3. Let $m \in k\left[x_{1}, \ldots, x_{d}\right]$ be a monomial. Then we call the smallest monomial ideal $I$ such that $I$ is p-Borel fixed and such that $m \in I$ the p-Borel principal ideal generated by $m$. We write $\langle m\rangle:=I$.

For the class of p-Borel principal ideals generated by a monomial of the form $x_{i}^{l}$, Aramova and Herzog find in [1] explicit minimal multigraded free resolutions. The goal of this section is to show that there also exist cellular minimal free multigraded resolutions for this class of monomial ideals. We produce these cellular resolutions by again applying Theorem 3.3.1 to the Taylor-resolution of the respective ideals.

Without loss of generality, we can assume that for $I=\left\langle x_{i}^{l}\right\rangle \subset k\left[x_{1}, \ldots, x_{d}\right]$ we have $i=d$. Let $l=\sum_{i=1}^{s} l_{i} p^{i}$ be the $p$-adic expansion of $l$. As usual, we denote by $\mathfrak{m}:=\left(x_{1}, \ldots, x_{d}\right)$ the maximal homogeneous ideal and write $\max (m):=\max \left\{i\right.$ s.t. $\left.x_{i} \mid m\right\}$ for monomials $m \in k\left[x_{1}, \ldots, x_{d}\right]$. To state the main result of this section, we define for a fixed ideal $I=\left\langle x_{d}^{l}\right\rangle$ for all $i=1, \ldots, d-1$ the following sets $\mathbf{S}_{0}(I), \ldots, \mathbf{S}_{d-1}(I)$ :

$$
\mathbf{S}_{i}(I):=\left\{\begin{array}{l|c}
\left(u_{t}, \ldots, u_{s},\left\{j_{0}, \ldots, j_{i-1}\right\}\right) & \begin{array}{c}
1 \leq t \leq s, \\
u_{r} \in \mathfrak{m}^{l_{r}} \text { for } r=t, \ldots, s, \\
1 \leq j_{1}<\ldots<j_{i-1}<\max \left(u_{t}\right)
\end{array}
\end{array}\right\}
$$

We set $\mathbf{S}(I):=\bigcup_{i=0}^{d-1} \mathbf{S}_{i}(I)$.

Theorem 4.5.4. Let $I=\left\langle x_{d}^{l}\right\rangle \subset k\left[x_{1}, \ldots, x_{d}\right]$ be the $p$-Borel principal ideal generated by $x_{d}^{l}$. There is a cellular minimal free resolution of $I$, the cells of dimension $i$ of which are in one-to-one correspondence with the elements of $\mathbf{S}_{i}(I)$

Proof. We prove the Theorem in three steps. Step 1 consists of constructing a matching on $X_{\text {Taylor }}(I)$, step 2 proves acyclicity of this matching and step 3 shows that the critical $i$-cells with respect to this matching are in one-to-one correspondence with the sets $\mathbf{S}_{i}(I)$.

Step 1: We construct maps

$$
\Phi: X_{\text {Taylor }^{(*)}}(I) \longrightarrow \mathbf{S}(I),
$$


and

$$
\Psi: \mathbf{S}(I) \longrightarrow X_{\text {Taylor }}{ }^{(*)}(I),
$$

such that $\Phi \circ \Psi=\operatorname{id}_{\mathbf{S}(I)}$ :

Construction of $\Phi: X_{\text {Taylor }}{ }^{(*)}(I) \longrightarrow \mathbf{S}(I):$ For $\tau \subset \operatorname{MinGen}(I)$ we define $\Phi(\tau)$ to be the unique $S=\left(u_{t}, \ldots, u_{s},\left\{j_{0}, \ldots, j_{i-1}\right\}\right) \in \mathbf{S}(I)$ satisfying

1. $\prod_{r>t} u_{r}^{p^{r}} \mid m$ for all $m \in \tau$,

2. There is no $u_{t} \in \mathfrak{m}^{l_{t}}$ such that $\prod_{r \geq t} u_{r}^{p^{r}} \mid m$ for all $m \in \tau$,

3. If $u \in \mathfrak{m}^{l_{t}}$ and $m \in \tau$ such that $u^{p^{t}} \prod_{r>t} u_{r}^{p^{r}} \mid m$, then

(a) $u \leq_{\text {revlex }} u_{t}$ and

(b) $\left.x_{j}^{a} \mid u, x_{j}^{a}\right\}\left\langle u_{t}\right.$ for some power $a \Rightarrow j \in\left\{j_{0}, \ldots, j_{i-1}\right\}$,

4. There exists $m \in \tau$ such that $\prod_{r \geq t} u_{r}^{p^{r}} \mid m$,

5. For all $j \in\left\{j_{0}, \ldots, j_{i-1}\right\}$ there exists $m \in \tau, u \in \mathfrak{m}^{l_{t}}$ and $a \in \mathbb{N}$ such that $u^{p^{t}} \prod_{r>t} u_{r}^{p^{r}} \mid m$ and $x_{j}^{a} \mid u$, but $x_{j}^{a} \mid u_{t}$.

Construction of $\Psi: \mathbf{S}(I) \longrightarrow X_{\text {Taylor }^{(*)}}(I):$ For

$$
S=\left(u_{t}, \ldots, u_{s},\left\{j_{0}, \ldots, j_{i-1}\right\}\right) \in \mathbf{S}_{i}(I)
$$

define $\Psi(S):=\left\{h_{0}, \ldots, h_{i}\right\} \subset \operatorname{MinGen}(I)$ by setting:

$$
\begin{aligned}
h_{k} & :=x_{\max \left(u_{t}\right)}^{\sum_{r<t} l_{r} p^{r}}\left(x_{j_{k}} / x_{\max \left(u_{t}\right)}\right)^{p^{t}} \prod_{r \geq t} u_{r}^{p^{r}}, j=0, \ldots, i-1, \\
h_{i} & :=x_{j_{0}}^{\sum_{r<t} l_{r} p^{r}} \prod_{r \geq t} u_{r}^{p^{r}}
\end{aligned}
$$

We make now another preparation for the construction of a suitable matching on $X_{\text {Taylor }}(I)$ :

For $\tau \in \operatorname{MinGen}(I)$ and $\Psi(\Phi(\tau)):=\left\{h_{0}, \ldots, h_{i}\right\} \subset \operatorname{MinGen}(I)$ as defined above, define $j_{\tau}$ to be the minimal integer, such that for all $j_{\tau} \leq j \leq i$ we have: 
1. $h_{j} \in \tau$,

2. $\Phi\left(\tau-\left\{h_{j}\right\}\right) \neq \Phi(\tau)$.

We obtain the following fact for all $\tau \subset \operatorname{MinGen}(I)$ such that $j_{\tau} \neq 0$ :

$$
\Phi\left(\tau-\left\{h_{j_{\tau}-1}\right\}\right)=\Phi\left(\tau \cup\left\{h_{j_{\tau}-1}\right\}\right) .
$$

Proof: If $h_{j_{\tau}-1} \in \tau$ the equation is obvious from the definition of $j_{\tau}$. But if $h_{j_{\tau}-1} \notin \tau$ checking the list of conditions shows that $\Phi(\tau)$ fullfills all required conditions to equal $\Phi\left(\tau \cup\left\{h_{j_{\tau}-1}\right\}\right)$ because of the definition of $h_{j_{\tau}-1}$.

Construction of the matching: Now we are ready to define the following matching $A \subset G_{X_{\text {Taylor }(I)}}$ : For all $\tau \in \operatorname{MinGen}(I)$ such that $j_{\tau} \neq 0$, with $\Psi(\Phi(\tau)):=\left\{h_{0}, \ldots, h_{i}\right\} \subset \operatorname{MinGen}(I)$ as defined above, we match

$$
\tau \cup\left\{h_{j_{\tau}-1}\right\} \rightarrow \tau-\left\{h_{j_{\tau}-1}\right\} .
$$

Equation 4.5.4.2 implies that this indeed is a matching.

Step 2: We show acyclicity of the above defined matching $A$ by verifying the conditions of Proposition 4.1.1:

Define

$$
P:=\left\{(S, \alpha) \mid S \in \mathbf{S}(I), \alpha \in \mathbb{Z}^{d}\right\}
$$

Endow $P$ with the structure of a poset by setting

$$
(S, \alpha) \leq\left(S^{\prime}, \alpha^{\prime}\right): \Leftrightarrow \tau_{S} \subset \tau_{S^{\prime}} \text { and } \alpha \leq \alpha^{\prime} .
$$

Here, for $S \in \mathbf{S}(\mathbf{I}), \tau_{S}$ is defined to be the maximal element $\tau \in X_{\text {Taylor }}(I)^{(*)}$ such that $\Phi(\tau)=S$. Such maximal elements exist because for $\tau, \tau^{\prime} \in$ $X_{\text {Taylor }}(I)^{(*)}$, such that $\Phi(\tau)=\Phi\left(\tau^{\prime}\right)$, we have $\Phi\left(\tau \cup \tau^{\prime}\right)=\Phi(\tau)$.

Define a map $f: X_{\text {Taylor }}{ }^{(*)}(I) \rightarrow P$ by setting

$$
f(\tau):=(\Phi(\tau), \operatorname{lcm}(\tau)) .
$$

Since

$$
\operatorname{lcm}\left(\tau-\left\{h_{j_{\tau}-1}\right\}\right)=\operatorname{lcm}\left(\tau \cup\left\{h_{j_{\tau}-1}\right\}\right)
$$


we have $A=\bigcup_{p \in P} A_{p}$, where $A_{p}$ is the restriction of the above matching to $f^{-1}(p)$. The definition of the map $\Phi$ and that of the $\tau_{S}, S \in \mathbf{S}$ imply that $f$ is order preserving. We now check that the $A_{p}, p \in P$ are acyclic: A cycle within $f^{-1}(p), p=(S, \alpha)$ must consist of adding and removing some elements of $\Psi(S)$. But removing some such element does not change the value of $j_{\tau}$, so it is always the same $h_{j_{\tau}-1}$ that could be added via a matching, leaving no possibility for a cycle. We have checked the conditions of Proposition 4.1.1 and have thereby proven acyclicity of $A$.

Step3: We show that the critical $i$-cells with respect to the matching $A$ are in one-to-one correspondence with the elements of $\mathbf{S}(\mathbf{I})_{\mathbf{i}}$ : The only situation in which a simplex $\tau$ is not matched, is when $j_{\tau}=0$. This implies $\Psi(\Phi(\tau))=\tau$. Therefore, the critical $i$-cells are, via $\Psi$, in one-to-one correspondence with the elements of $\mathbf{S}(\mathbf{I})_{\mathbf{i}}$.

\subsection{Application to the Resolution of $k$ over Semigroup-Rings}

For an affine semigroup $\Lambda \subseteq \mathbb{N}^{d}$ and $\lambda \in \Lambda$ we denote by $\Delta(\underline{\mathbf{0}}, \lambda)$ the order complex of the open interval $(\underline{\mathbf{0}}, \lambda)$ in the poset $\Lambda$. Note that

$$
\Delta(\underline{\mathbf{0}}, \lambda)=\Delta\left(\Lambda_{0}\right)_{\prec \lambda}
$$

for the ordercomplex $\Delta\left(\Lambda_{0}\right)$ (see Lemma 2.3.34).

Proposition 4.6.1. Let $\Lambda \subseteq \mathbb{N}^{d}$ be an affine semigroup such that $\Delta(\underline{\mathbf{0}}, \lambda)$ is shellable for all $\lambda \in \Lambda$. Then there is a cellular minimal free resolution of $k$ as an $R=k[\Lambda]$-module.

Proof. We set

$$
\Delta\left(\Lambda_{0}\right)_{\lambda}^{(*)}:=\left\{\sigma \in \Delta\left(\Lambda_{0}\right)^{(*)} \mid \operatorname{gr}(\sigma)=\lambda\right\}
$$

The maps

$$
\begin{aligned}
\Delta\left(\Lambda_{0}\right)_{\lambda}^{(*)} & \longrightarrow \Delta(\underline{\mathbf{0}}, \lambda)^{(*)} \\
\left(\lambda_{0}, \ldots, \lambda_{i-1}, \lambda_{i}=\lambda\right) & \longmapsto\left(\lambda_{0}, \ldots, \lambda_{i-1}\right)
\end{aligned}
$$


are isomorphisms of posets for all $\lambda \in \Lambda$. According to Theorem 4.1.6, since $\Delta(\underline{\mathbf{0}}, \lambda)$ is shellable there exists a matching $A_{\lambda}$ on $\Delta(\underline{\mathbf{0}}, \lambda)$ such that the number of critical $i$-cells is given by $\operatorname{dim} \tilde{H}_{i}(\Delta(\underline{\mathbf{0}}, \lambda))=\operatorname{dim} \tilde{H}_{i}\left(\Delta\left(\Lambda_{0}\right)_{\prec \lambda}\right)$. The above maps carry these matchings $A_{\lambda}$ over to matchings $\tilde{A}_{\lambda}$ on $\Delta\left(\Lambda_{0}\right)_{\lambda}^{(*)}$. By Proposition 4.1.1

$$
A:=\bigcup_{\lambda \in \Lambda} \tilde{A}_{\lambda}
$$

is again an acyclic matching and the number of critical $i$-cells of multidegree $\lambda$ is given by $\operatorname{dim} \tilde{H}_{i-1}\left(\Delta\left(\Lambda_{0}\right)_{\prec \lambda}\right)$. By Proposition 2.2.5 these are also the Betti numbers of the minimal free multigraded resolution of $k$ as $k[\Lambda]$-module, so the cellular resolution supported by the Morse complex $\Delta\left(\Lambda_{0}\right)_{A}$ is minimal.

Remark 4.6.2. The above Proposition and Theorem 4.1.8 share the following background: Given a cellular $\mathbb{Z}^{d}$-graded resolution of a module $M$ supported by a CW-complex $X$ such that for all $\alpha \in \mathbb{Z}^{d}$ the subcomplex $X_{\prec \alpha}$ is shellable and such that the partially ordered sets $X_{\prec \alpha}^{(*)}$ and $X_{\alpha}^{(*)}:=\left\{\sigma \in X^{(*)} \mid \operatorname{gr}(\sigma)=\right.$ $\alpha\}$ are isomorphic, there exists a cellular minimal free resolution of $M$.

Proof. The proof is the same as for Proposition 4.6.1. 


\section{Bibliography}

[1] Aramova, A., Herzog, J.: p-Borel principal ideals. Illinois J. of Math. 41 (1997), 103-121.

[2] Aramova, A., Herzog, J., Hibi, T.: Squarefree lexsegment ideals. Math. Z. 228 (1998), 353-378.

[3] Batzies, E., Welker, V.: Discrete Morse theory for cellular resolutions. J. reine angew. Math. 543 (2002), 147-168.

[4] Bayer, D., Peeva, I., Sturmfels, B.: Monomial resolutions. Math. Res. Lett. 5 (1998), 31-46.

[5] Bayer, D., Sturmfels, B.: Cellular resolutions of monomial modules.

[6] Björner A., Wachs M.: Shellable non-pure complexes and posets I. Trans. Amer. Math. Soc. 348 (1996), 1299-1327.

[7] Björner A., Ziegler G.: Broken circuit complexes: factorizations and generalizations. J. Combin. Theory Ser. B 51 (1991), 96-126.

[8] Bredon, G.E.: Topology and Geometry. Graduate Texts in Mathematics, 139. Springer-Verlag, New York, 1993.

[9] Chari, M.K.: On discrete Morse functions and combinatorial decompositions. Preprint (1996).

[10] Jonsson, J.: On the homology of some complexes of graphs. Preprint (1999). 
[11] Eisenbud, D.: Commutative Algebra with a View Toward Algebraic Geometry. Springer, New York (1996). J. Reine Angew. Math. 502 (1998), 123-140.

[12] Eliahou, S., Kervaire, M.: Minimal resolutions of some monomial ideals. J. Algebra 129 (1990), 1-25.

[13] Forman, R.: Morse theory for cell complexes. Adv. Math. 134 (1998), 90-145.

[14] Forman, R.: Combinatorial differential topology and geometry. New Perspectives in algebraic Combinatorics (Berkeley, CA. 1996-97). Math. Sci. Res. Inst. Publ. 38, Cambridge Univ. Press, Cambridge, 91999, 177-206.

[15] Herzog, J., Reiner, V., Welker, V.: Componentwise linear ideals and Golod rings.

[16] Lyubeznik, G.: A new explicit finite free resolution of ideals generated by monomials in an $R$-sequence.

[17] Munkres, J..: Elements of algebraic topology. Addison-Wesley Publishing Company (1984).

[18] Novik, I.: Lyubeznik's resolution and rooted complexes. Preprint (2001).

[19] Novik, I., Postnikov, A., Sturmfels, B.: Syzygies of oriented matroids, Preprint (2000).

[20] Peeva, I.: Strongly stable ideals. PhD-Thesis. Brandeis University (1994).

[21] Peeva, I., Reiner, V., Sturmfels, B.: How to shell a monoid. Math. Ann. 310 (1998), 379-393.

[22] Sköldberg: E-Mail communication, (2000). 
[23] Taylor, D.: Ideals generated by an $R$-sequence. PhD-Thesis. University of Chicago (1966).

[24] Welker, V., Ziegler, G., Zivaljevic, R.: Homotopy colimits - comparison lemmas for combinatorial applications. J. reine angew. Math. 509 (1999), 117-149. 


\section{Kapitel 5}

\section{Deutsche Zusammenfassung}

\section{$5.1 \quad$ Einleitung}

Diese Arbeit ist ein Beitrag zur kombinatorischen kommutativen Algebra, und zwar zur Theorie der zellulären multigraduierten Auflösungen monomialer Moduln.

Aus der linearen Algebra kennt man das grundlegende Konzept der linearen Abhängigkeit. Kennzeichnend ist die Tatsache, dass jeder Vektorraum $V$ über einem Körper $k$ ein freier $k$-Modul ist. Insbesondere sind Vektoren $v_{1}, \ldots, v_{n} \in V$ genau dann maximal linear unabhängig, wenn sie minimal erzeugen.

Das Konzept der Abhängigkeit von Polynomen $p_{1}, \ldots, p_{n} \in k\left[x_{1}, \ldots, x_{d}\right]$ über dem Polynomring $k\left[x_{1}, \ldots, x_{d}\right]$ ist sehr viel komplexer. Zum Beipiel sind hier maximale Unabhängigkeit und minimales Erzeugen nicht äquivalent.

Ein Mass für die Abhängigkeit sind (minimale) freie Auflösungen des von den Polynomen $p_{1}, \ldots, p_{n}$ erzeugten Ideals $I=\left(p_{1}, \ldots, p_{n}\right)$. Allerdings sind diese sehr schwierig zu konstruieren. Selbst das Problem der Konstruktion minimaler freier Auflösungen monomialer Ideale (das sind Ideale, die von Monomen erzeugt werden) ist nicht gelöst.

Ein vielversprechender Zugang zu diesem Problem ist das Studium sogenannter zellulärer Aufösungen. Ist das aufzulösende Ideal $\mathbb{Z}^{d}$-graduiert, so entstehen diese durch Homogenisieren des zellulären Kettenkomplexes eines $\mathbb{Z}^{d}$-graduierten CW-Komplexes. 
Das klassische Beispiel einer zellulären $\mathbb{Z}^{d}$-graduierten freien Auflösung ist die Taylor-Auflösung. Der zugrundeliegende $\mathbb{Z}^{d}$-graduierte CW-Komplex ist hier der Standard Simplex $\Delta_{n}$, dessen Ecken die monomialen Erzeuger des Ideals $I$ bilden. Die $\mathbb{Z}^{d}$-Graduierung erfolgt durch das kleinste gemeinsame Vielfache, das heisst der Multigrad $\alpha \in \mathbb{Z}^{d}$ eines Simplex $\sigma=\left\{m_{i_{1}}, \ldots, m_{i_{k}}\right\}$ $\in \Delta_{n}$ ist dasjenige $\alpha$ mit $\underline{\mathbf{x}}^{\alpha}=\operatorname{kgV}\left\{m_{i_{1}}, \ldots, m_{i_{k}}\right\}$.

\section{2 Überblick über die Arbeit}

Im Allgemeinen sind zelluläre Auflösungen wie die Taylor-Auflösung bei weitem nicht minimal. Die vorliegende Arbeit liefert eine allgemeine Methode zur Minimalisierung gegebener zellulärer Auflösungen. Wir verwenden hierfür als Hilfsmittel aus der kombinatorischen Topologie die diskrete Morsetheorie. Die Hauptaussage dieser von Forman [13, 14] entwickelten Theorie ist wie folgt (wir verwenden die Theorie in einer Version von Chari $[9])$ :

Hat man einen CW-Komplex $X$ und ein azyklisches Matching $A$ auf dem gerichteten Zellgraphen von $X$, so existiert ein zu $X$ homotopieäquivalenter CW-Komplex $X_{A}$ (der sogenannte Morsekomplex), dessen Zellen in Bijektion zu den $A$-kritischen Zellen von $X$ stehen. Diesen Übergang von $X$ zu $X_{A}$ kann man ansehen als Übergang zu einer effektiveren CW-Zerlegung von $X$.

Die zentrale neue Idee dieser Arbeit besteht darin, diesen Prozess von CW-Komplexen auf zelluläre Aufösungen zu übertragen. Wir zeigen das folgende: Hat man eine zelluläre $\mathbb{Z}^{d}$-graduierte freie Auflösung eines Moduls $M$ mit zugrundeliegendem $\mathbb{Z}^{d}$-graduiertem CW-Komplex $X$ und ein azyklisches Matching $A$ auf dem gerichteten Zellgraphen von $X$, so liefert der Morsekomplex $X_{A}$ seinerseits eine $\mathbb{Z}^{d}$-graduierte freie Auflösung desselben Moduls $M$. Da der CW-Komplex $X_{A}$ aus weniger Zellen besteht als $X$, ist die Auflösung, die aus $X_{A}$ entsteht, kleiner als die ursprüngliche, die aus $X$ entstand.

Ein Grossteil der Arbeit besteht darin, durch Anwendung dieses Ergebnisses minimale $\mathbb{Z}^{d}$-graduierte freie Auflösungen für gewisse Klassen monomialer Moduln zu konstruieren. Diese beinhalten alle generischen, stabilen und quadratfrei-stabilen monomialen Ideale, alle Stanley-Reisner Ideale, deren 
zugehöriger simplizialer Komplex ein schälbares Alexanderdual besitzt, sowie eine sehr spezielle Klasse von p-Borel-fixed Idealen. In einer weiteren Anwendung präsentieren wir unter speziellen Vorraussetzungen an eine affine Halbgruppe $\Lambda$ minimale $\mathbb{Z}^{d}$-graduierte freie Auflösungen des Körpers $k$ über dem Halbgruppenring $k[\Lambda]$.

Die Idee, diskrete Morsetheorie auf zelluläre Aufösungen anzuwenden, wurde bereits in [3] vorgestellt. Hierbei handelt es sich um eine gemeinsame Veröffentlichung mit Volkmar Welker.

\subsection{Struktur der Arbeit}

\subsubsection{Kapitel 1}

Das 1. Kapitel enthält die Einleitung. Diese ist inhaltlich ähnlich dieser Zusammenfassung in deutscher Sprache.

\subsubsection{Kapitel 2}

Das 2. Kapitel besteht aus Definitionen, elementaren Tatsachen über und Beispielen von zellulären $\mathbb{Z}^{d}$-graduierten freien Auflösungen.

\section{Abschnitt 2.1}

In diesem Abschnitt werden folgende mathematischen Objekte definiert:

1. Affine Halbgruppenringe $k[\Lambda]$,

2. $\mathbb{Z}^{d}$-graduierte freie Kettenkomplexe,

3. $\mathbb{Z}^{d}$-graduierte freie Auflösungen,

4. Minimalität $\mathbb{Z}^{d}$-graduierter freier Auflösungen,

5. CW-Komplexe,

6. zelluläre Homologie,

7. simpliziale Komplexe, 
8. $\mathbb{Z}^{d}$-graduierte CW-Komplexe,

9. zelluläre Kettenkomplexe und zelluläre Auflösungen,

10. monomiale Moduln und deren Eigenschaft, co-Artinsch zu sein, sowie

11. ganzzahlige Gitter.

Wir geben hier ausschnittsweise die wichtigsten dieser Definitionen wieder:

Definition 5.3.1 ( $\mathbb{Z}^{d}$-graduierte freie Auflösung). Es sei $R=k[\Lambda]$ ein affiner Halbgruppenring, $M=\bigoplus_{\alpha \in \mathbb{Z}^{d}} M_{\alpha}$ ein $\mathbb{Z}^{d}$-graduierter $R$-Modul. Eine $\mathbb{Z}^{d}$-graduierte freie Auflösung $\mathcal{F}$ von $M$ ist ein $\mathbb{Z}^{d}$-graduierter freier Kettenkomplex

$$
\mathcal{F}=\ldots \stackrel{\partial_{i+1}}{\longrightarrow} \mathcal{F}_{i} \stackrel{\partial_{i}}{\longrightarrow} \mathcal{F}_{i-1} \stackrel{\partial_{i-1}}{\longrightarrow} \ldots \stackrel{\partial_{1}}{\longrightarrow} \mathcal{F}_{0}
$$

über $R$ mit den folgenden Eigenschaften:

1. $\mathcal{F}$ ist exakt, das heisst,

$$
\operatorname{Ker} \partial_{i}=\operatorname{Im} \partial_{i+1} \text { für alle } i \in \mathbb{N} \backslash\{0\},
$$

2. Coker $\partial_{1} \cong M$.

Definition 5.3.2. Ein topologischer Raum heisst (offene) Zelle der Dimension $d$ (bzw $d$-Zelle) falls er homöomorph ist zur $d$-dimensionalen offenen Kugel

$$
\stackrel{\circ}{B^{d}}=\left\{x=\left(x_{1}, \ldots, x_{d}\right) \in \mathbb{R}^{d} \mid \sum_{i=1}^{d} x_{i}^{2}<1\right\} .
$$

Definition 5.3.3 (CW-complex). Wir nennen einen topologischen Raum $X$ einen $C W$-Komplex, falls es eine Menge $X^{(*)}=\left\{\sigma_{i} \mid i \in I\right\}$ disjunkter offener Zellen gibt, so dass gilt:

1.

$$
X=\bigcup_{i \in I} \sigma_{i}
$$


2. $X$ ist Hausdorffsch,

3. für jede offene Zelle $\sigma \in X^{(*)}$ der Dimension $d$ gibt es eine stetige Abbildung

$$
f_{\sigma}: B^{d} \longrightarrow X
$$

so dass die Einschränkung $\stackrel{\circ}{f_{\sigma}}:=\left.f_{\sigma}\right|_{B^{d}}$ ein Homöomorphismus

$$
\stackrel{\circ}{f}_{\sigma}: \stackrel{\circ}{ }^{d} \stackrel{\cong}{\longrightarrow} \sigma
$$

ist und so dass $f_{\sigma}\left(S^{d-1}\right)$ nur mit endlich vielen Zellen einen nichtleeren Schnitt besitzt. Alle diese Zellen haben höchstens die Dimension $d-1$,

4. eine Teilmenge $A \subset X$ ist in $X$ abgeschlossen, genau dann wenn $A \cap \bar{\sigma}$ in $\bar{\sigma}$ abgschlossen ist für alle $\sigma \in X^{(*)}$.

Definition 5.3.4 (Graduierter CW-komplex). 1. Es sei $(P, \preceq)$ eine partiell geordnete Menge, $(X, A)$ ein CW-Paar und $f:(X, A)^{(*)} \rightarrow P$ eine Abbildung. Wir nennen $(X, A, f)$ ein $P$-graduiertes Paar, falls $f$ ordnungserhaltend ist.

2. Ist $(X, f):=(X, \emptyset, f)$ ein $P$-graduierter CW-Komplex und $p \in P$, so bezeichnen wir mit $X_{\preceq p}$ den $P$-graduierten Unter-CW-Komplex, der aus all denjenigen Zellen $\sigma \in X^{(*)}$ besteht, für die gilt: $f(\sigma) \preceq p$.

Definition 5.3.5 (Zelluläre Auflösung). Es sei $R=k[\Lambda]$ ein affiner Halbgruppenring. Wir nennen eine $\mathbb{Z}^{d}$-graduierte Auflösung

$$
\mathcal{F}=\ldots \mathcal{F}_{i} \stackrel{\partial_{i}}{\longrightarrow} \mathcal{F}_{i-1} \stackrel{\partial_{i-1}}{\longrightarrow} \ldots \stackrel{\partial_{1}}{\longrightarrow} \mathcal{F}_{0}, \mathcal{F}_{i}=\bigoplus_{\alpha \in \mathbb{Z}^{d}} F_{i}^{\alpha}, F_{i}^{\alpha}=R(-\alpha)^{\beta_{i}^{\alpha}}
$$

zellulär falls ein $\left(\mathbb{Z}^{d}, \Lambda\right)$-graduiertes CW-Paar $(X, A$, gr) existiert, so dass gilt:

(a) Es existiert eine Basis $e_{\sigma}$ von $C_{i}^{\alpha}$ indiziert mit $i$-Zellen $\sigma$ aus $(X, A)^{(i)}$ mit der Eigenschaft $\operatorname{gr}(\sigma)=\alpha$. 
(b) Für $\sigma \in(X, A)^{(i)}$ gilt:

$$
\partial_{i} e_{\sigma}=\sum_{\sigma \geq \sigma^{\prime} \in X^{(i-1)}}\left[\sigma^{\prime}: \sigma\right] \underline{\mathbf{x}}^{\operatorname{gr}(\sigma)-\operatorname{gr}\left(\sigma^{\prime}\right)} e_{\sigma^{\prime}}, i \geq 1
$$

wobei $\left[\sigma^{\prime}: \sigma\right]$ der Koeffizient von $\sigma^{\prime}$ im Differential von $\sigma$ im zellulären Kettenkomplex von $(X, A)$ ist.

\section{Abschnitt 2.2}

In diesem Abschnitt werden einige Grundlegende Tatsachen über zelluläre freie Auflösungen aufgeführt:

1. Es wird ein Kriterium von Bayer und Sturmfels ([5]) zitiert, welches besagt, dass ein $\mathbb{Z}^{d}$-graduierter $C W$-Komplex $X$ genau dann eine zelluläre Auflösung unterstützt, wenn gilt, dass alle Unterkomplexe $X_{\preceq \alpha}, \alpha \in \mathbb{Z}^{d}$ azyklisch oder leer sind.

2. Es wird die allgemein bekannte Tatsache gezeigt, dass sich die BettiZahlen der minimalen $\mathbb{Z}^{d}$-graduierten freien Auflösung eines Moduls $M$ aus jeder $\mathbb{Z}^{d}$-graduierten freien Auflösung $\mathcal{F}$ dieses Moduls vermöge der folgenden Formel berechnen lassen:

$$
\beta_{i}^{\alpha}(M)=\operatorname{dim} H_{i}\left(\left(\mathcal{F}^{\prec \alpha}\right)_{\alpha},\left(\mathcal{F}^{\prec \alpha}\right)_{\alpha}\right) .
$$

3. Es wird gezeigt, dass auf ähnliche Weise die Betti-Zahlen der minimalen $\mathbb{Z}^{d}$-graduierten freien Auflösung eines Moduls $M$ sich auch aus der Homologie eines einer zellulären Auflösung von $M$ zugrundeliegenden graduierten CW-Paares $(X, A)$ berechnen lassen. Die Formel ist:

$$
\begin{gathered}
\beta_{i}^{\alpha}(M)=\operatorname{dim} H_{i}\left(X_{\preceq \alpha}, X_{\prec \alpha} \cup A_{\preceq \alpha} ; k\right) \\
=\left\{\begin{array}{ll}
\operatorname{dim} H_{i-1}\left(X_{\prec \alpha}, A_{\prec \alpha} ; k\right) & \text { falls } A_{\preceq \alpha} \neq \emptyset \\
\operatorname{dim} \tilde{H}_{i-1}\left(X_{\prec \alpha} ; k\right) & \text { falls } A_{\preceq \alpha}=\emptyset
\end{array},\right.
\end{gathered}
$$

wobei die erste Gleichung für alle $i \geq 0$ gilt und die zweite für alle $i \geq 2$. 
4. Es wird gezeigt: Falls das graduierte CW-Paar $(X, A)$ eine graduierte zelluläre freie Auflösung des Moduls $M$ unterstützt, $Y \subset X$ ein Unterkomplex von $Y$ ist, der eine graduierte zelluläre freie Auflösung des Untermoduls $N \subset M$ unterstützt, dann unterstützt $(X, Y)$ eine graduierte zelluläre freie Auflösung des Moduls $M / N$.

\section{Abschnitt 2.3}

In diesem Abschnitt werden Beispiele graduierter zellulärer freier Auflösungen aufgeführt. Bekannte Beispiele sind:

1. Taylor-Auflösung [23],

2. Scarf-Auflösung [4],

3. Hull-Auflösung [5],

4. Lyubeznik's Auflösung [16],

5. Auflösungen via Wurzelkomplexen (rooted complexes) [18] und

6. Bar-Auflösungen.

Als neue Klassen graduierter zellulärer freier Auflösungen werden aufgeführt:

1. LCM-Auflösungen,

2. $\mathrm{LCM}^{*}$-Auflösungen und

3. hypersimpliziale Auflösungen für Potenzen des maximalen homogenen Ideals im Polynomring $k\left[x_{1}, \ldots, x_{d}\right]$.

Die LCM-und LCM*-Auflösungen eines monomialen Modules $M$ entstehen aus dem Ordnungskomplex über dem $L C M$-Verbandes von $M$ bzw eines vom $L C M$-Verband abgeleiteten Verbandes. Die hypersimplizialen Auflösungen für Potenzen des maximalen homogenen Ideals entstehen aus einer zellulären Würfelstruktur im $\mathbb{R}^{d}$ und ihrer hypersimplizialen Unterteilung. 


\subsubsection{Kapitel 3}

In diesem Kapitel wird Formans diskrete Morsetheorie vorgestellt und für unsere Bedürfnisse erweitert. Die entscheidende Aussage ist:

Theorem 5.3.6. Es sei $R=k[\Lambda]$ ein affiner Halbgruppenring und $M$ ein $\mathbb{Z}^{d}$-graduierter $R$-Modul. Es sei $(X$, gr $)$ ein kompakt $\left(\mathbb{Z}^{d}, \Lambda\right)$-graduierter $C W$ Komplex der die zelluläre Auflösung $\mathcal{F}_{X}^{\mathrm{gr}}$ von $M$ unterstütze. Weiterhin sei $A$ ein azyklisches Matching auf $X$. Dann unterstützt der $\left(\mathbb{Z}^{d}, \Lambda\right)$-graduierte $C W$-Komplex $\left(X_{A}\right.$, gr) eine zelluläre Auflösung $\mathcal{F}_{X_{A}}^{\mathrm{gr}}$ von $M$.

Hierbei assoziieren wir zu $X$ den gerichteten Graphen $G_{X}$, dessen Eckenmenge die Menge der Zellen von $X$ ist und dessen Kantenmenge aus all denjenigen $\tau \rightarrow \sigma$ besteht, wo $\sigma$ maximale Seite von $\tau$ ist. Ein azyklisches Matching $A$ auf $X$ ist gegeben durch eine Menge von Paaren $(\tau, \sigma)$ von Zellen von $X$, derart dass

1. $\sigma$ ist reguläre Seite von $\tau$ für alle Paare $(\tau, \sigma)$ aus $A$,

2. keine Zelle $\sigma$ kommt in zwei verschiedenen Paaren von $A$ vor und

3. der gerichtete Graph $G_{X}^{A}$, der aus $G_{X}$ durch Umdrehen der Richtung aller Kanten aus $A$ entsteht, ist azyklisch.

Gemäss den Resultaten von Forman ist der Morsekomplex $X_{A}$ wiederum ein CW-Komplex und seine Zellen stehen in Bijektion mit den sogenannten kritischen Zellen von $X$, das sind diejenigen Zellen von $X$, die in keinem der Parre von $A$ vorkommen.

Inhalt des 3. Kapitels ist, neben der Darstellung von Formans Ergebnissen:

1. Die Verallgemeinerung dieser Ergebnisse auf graduierte CW-Komplexe und

2. der Beweis (im Abschnitt 3.4 über Morsedifferentiale), dass es sich bei dem von Forman beschriebenen differentiellen Morsekomplex um die Homologiesequenz des (topologischen) Morsekomplexes $X_{A}$ handelt. Dies liefert uns im Folgenden die Möglichkeit, Differentiale in zellulären Auflösungen, die von Morsekomplexen unterstützt sind, explizit zu bestimmen. 


\subsubsection{Kapitel 4}

In diesem Kapitel wenden wir die diskrete Morsetheorie auf konkrete zelluläre Auflösungen an und erhalten auf diese Weise minimale zelluäre Auflösungen für mehrere Klassen von Moduln:

1. In Abschnitt 4.1 zeigen wir, dass für schälbare monomiale Moduln $M$ mit geeigneten Matchings aus der $L C M^{*}$-Auflösung eine zelluläre minimale Auflösung konstruiert werden kann.

2. In Abschnitt 4.2 konstruieren wir eine allgemeine Methode, aus der Taylor Aufösung eines co-Artischen monomialen Moduls $M$ und einer Menge linearer Ordnungen auf den Erzeugern von $M$ mit gewissen Voraussetzungen eine neue zelluläre Auflösung zu erhalten. Diese Methode kann als Verallgemeinerung von der Auflösung von Lyubeznik angesehen werden. Wir zeigen, dass auf diese Weise für generische coArtinsche monomiale Moduln und wiederum für schälbare co-Artinsche monomiale Moduln zelluläre minimale Aufösungen konstruiert werden können.

3. Abschnitt 4.3 wendet die diskrete Morsetheorie auf die hypersimpliziale Auflösung der Potenzen des maximalen homogenen Ideals an und erhält auch hier eine zelluläre minimale Auflösung.

4. Abschnitt 4.4 zeigt, dass die Auflösungen via Wurzelkomplexen sich durch Anwendung der diskreten Morsetheorie auf die Taylorauflösung realisieren lassen.

5. In Abschnitt 4.5 wird gezeigt, dass für "p-Borel fixed"-Ideale, die von einem Monom der Form $x_{d}^{l}$ (im geeigneten Sinne) erzeugt werden, ebenfalls eine zelluläre minimale Auflösung durch Anwendung der diskreten Morsetheorie auf die Taylorauflösung erhalten werden kann.

6. Zuletzt wird in Abschnitt 4.6 gezeigt, dass für einen Halbgruppenring $k[\Lambda]$ mit der Eigenschaft, dass alle Ordnungskomplexe $\Delta(\underline{\mathbf{0}}, \lambda)$ über offenen Intervallen $(\underline{\mathbf{0}}, \lambda)$ schälbar sind, der Körper $k$ eine zelluläre minimale Auflösung über $k[\Lambda]$ besitzt. 